Supporting information

\title{
Changes in healthy human IgG Fc-glycosylation after birth and during early childhood
}

\author{
Noortje de Haan ${ }^{1}$, Karli R. Reiding ${ }^{1}$, Gertjan Driessen ${ }^{2}$, Mirjam van der Burg ${ }^{3}$ and Manfred Wuhrer ${ }^{1, *}$ \\ ${ }^{1}$ Leiden University Medical Center, Center for Proteomics and Metabolomics, Leiden, The Netherlands \\ ${ }^{2}$ Erasmus MC, Pediatrics, Rotterdam, The Netherlands \\ ${ }^{3}$ Erasmus MC, Immunology, Rotterdam, The Netherlands
}

*Correspondence: Manfred Wuhrer, Leiden University Medical Center, Center for Proteomics and Metabolomics, P.O. Box 9600, 2300 RC Leiden, The Netherlands; m.wuhrer@lumc.nl; Tel: +31-71-5268744

\section{Content}

In this document:

Figure S-1 Data quality

Figure S-2 Glycans and glycan traits plotted versus age 0.1 to 17 , separated for sex

Figure S-3 Glycans and glycan traits plotted versus age 0.1 to 17 , sexes combined

Figure S-4 Glycans and glycan traits per age class, separated for sex

Figure S-5 Glycans and glycan traits per age class, sexes combined

In the supporting .xlsx file:

Table S-1: Derived trait calculations

Table S-2: Correlation coefficients between the IgG Fc-glycosylation features and age

Table S-3: Sex differences in IgG Fc N-glycosylation per age class

Table S-4: Comparison between IgG Fc-glycosylation found in umbilical cord samples and in the youngest age category

Table S-5: Comparison between IgG Fc-glycosylation found in umbilical cord samples and in the oldest age category

Table S-6: Overview of the relevant literature in which current results are embedded 


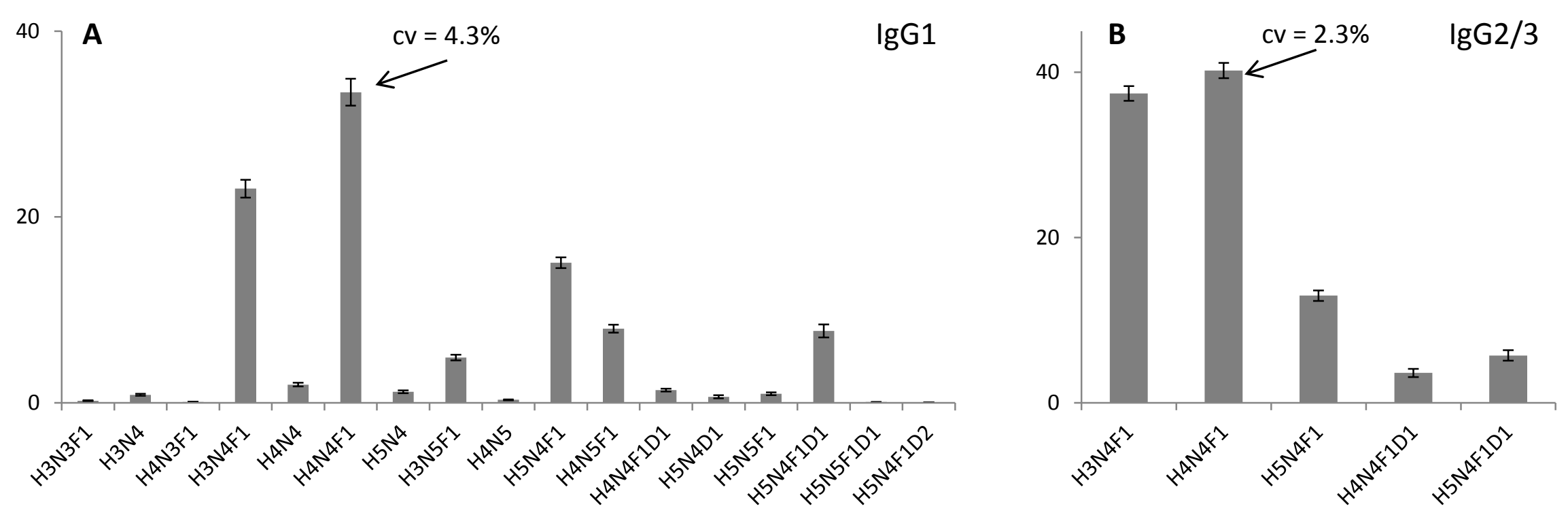

Figure S-1 Average relative abundances of A) IgG1 and B) IgG2/3 glycopeptides in respectively 29 and 28 pooled plasma samples. A CV of only $4.3 \%$ was observed for the most abundant glycoform on $\operatorname{lgG} 1$ and of $2.3 \%$ for the most abundant glycoform on IgG2/3. The error bars represent the standard deviation over the measurements. 
Figure S-2 Levels of all glycans and derived glycan traits of IgG1 and IgG2/3, separated for sex. Pink circles: female, blue diamonds: male, $r$ : correlation coefficient. The linear lines were fitted through female and male samples separately and are not related to the calculated correlation coefficient. After Bonferroni correction, $p$-values below $8.7 \mathrm{E}-5$ were considered statistically significant.

\section{lgG1_H3N3F1}

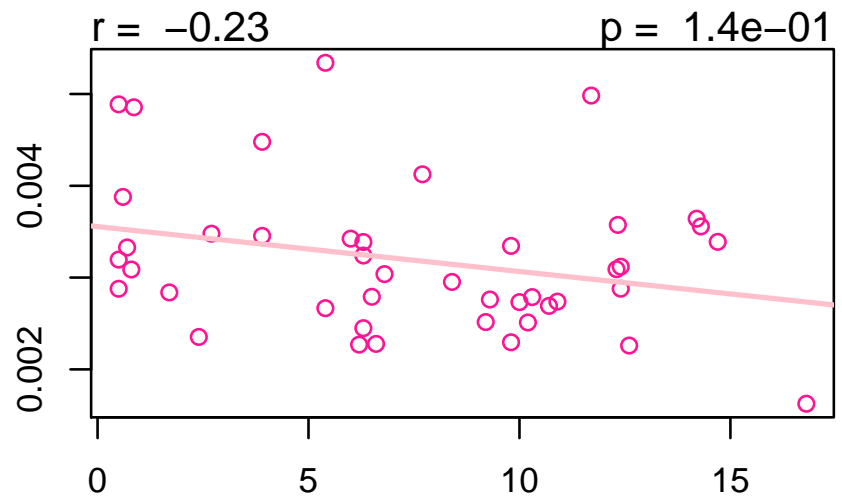

Age (years)

\section{IgG1_H3N3F1}

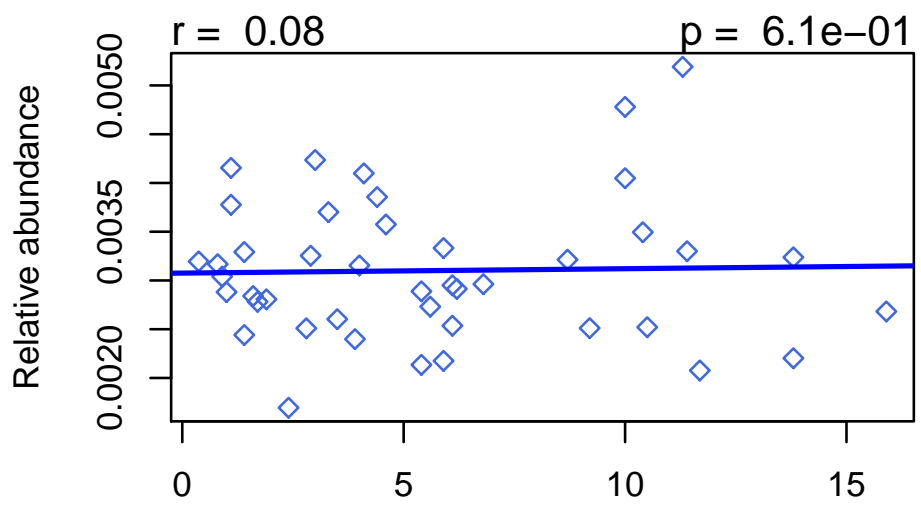


IgG1_H3N4

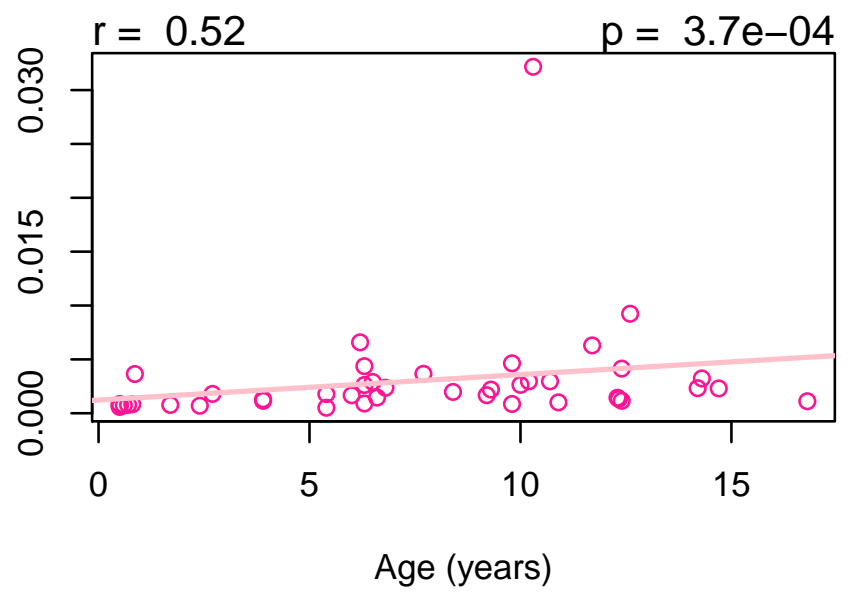

IgG1_H4N3F1

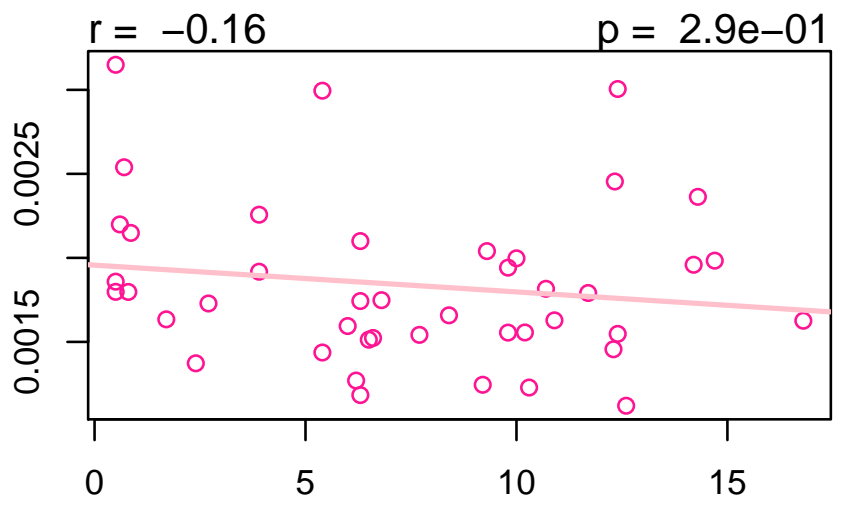

Age (years)

\section{lgG1_H3N4}

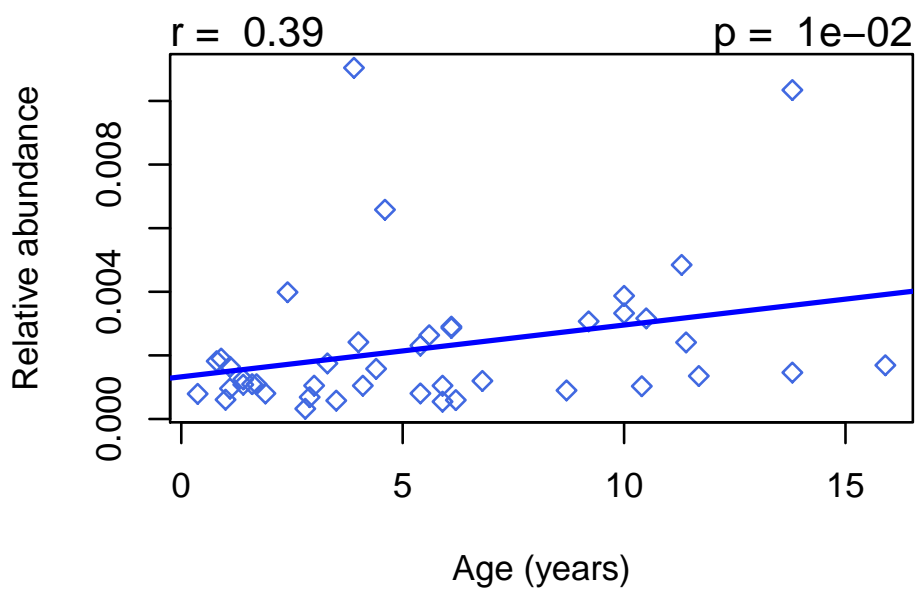

\section{IgG1_H4N3F1}

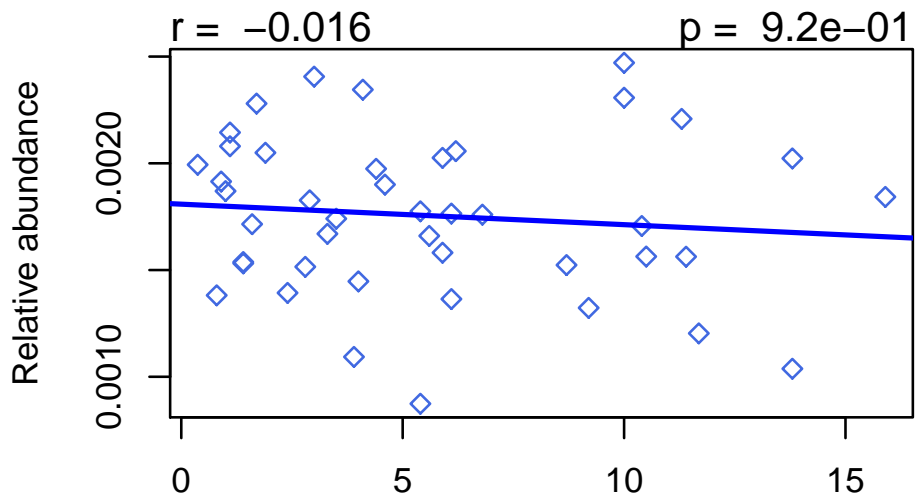

Age (years) 


\section{IgG1_H3N4F1}

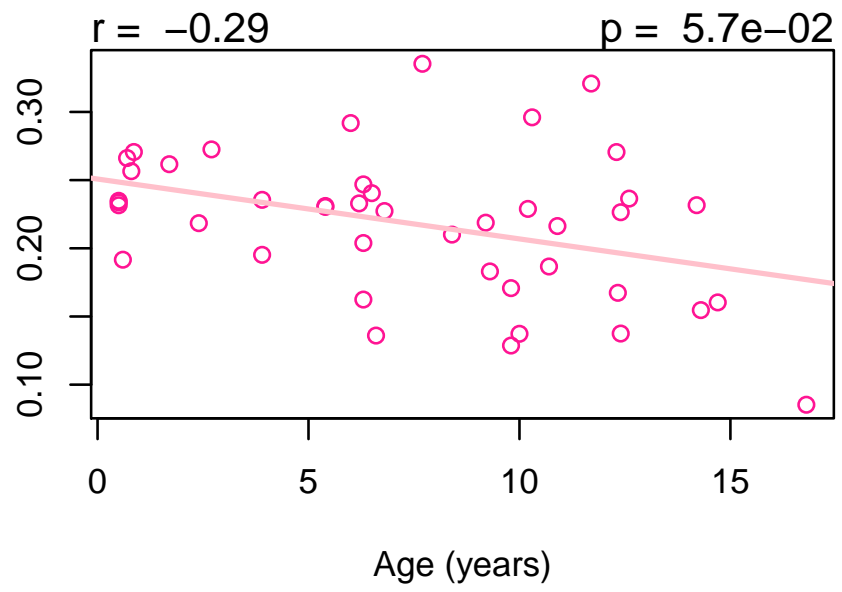

IgG1_H4N4

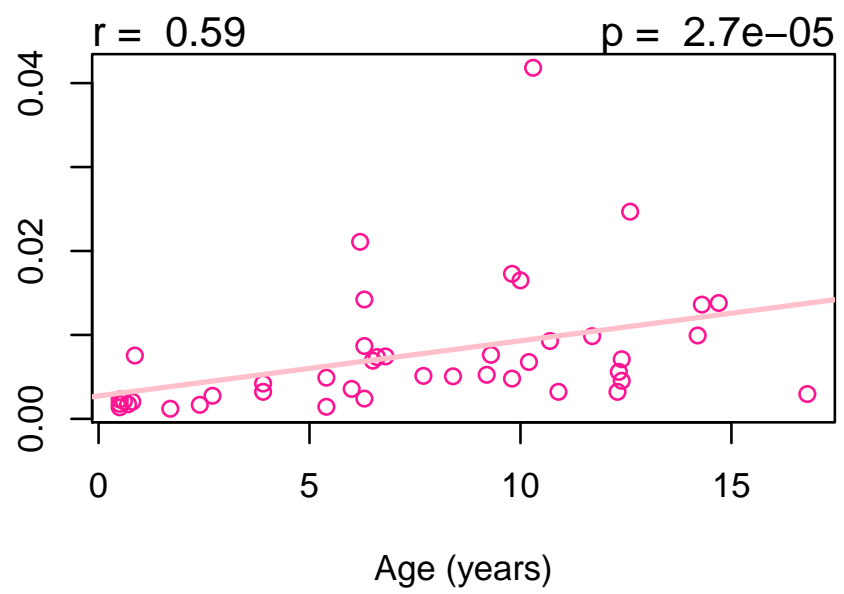

\section{IgG1_H3N4F1}

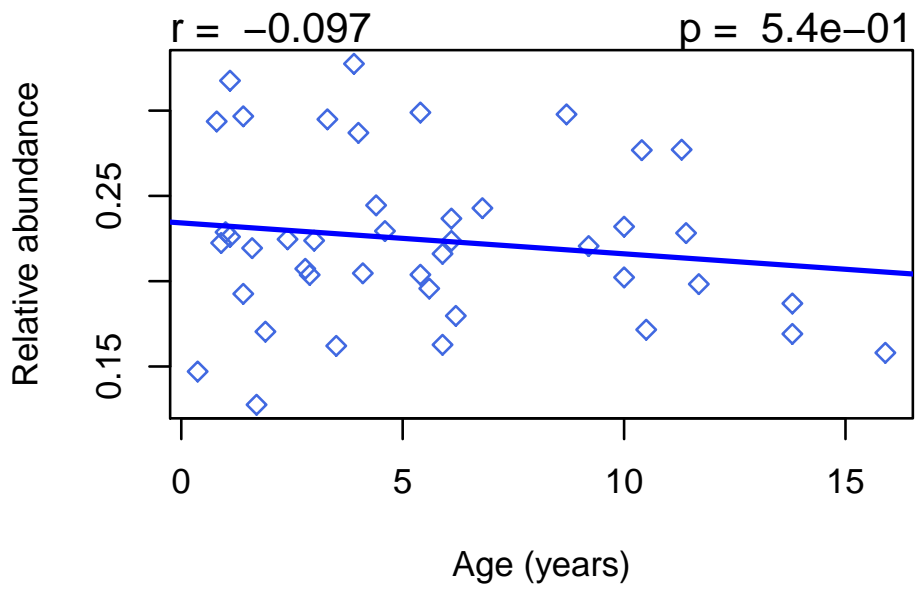

IgG1_H4N4

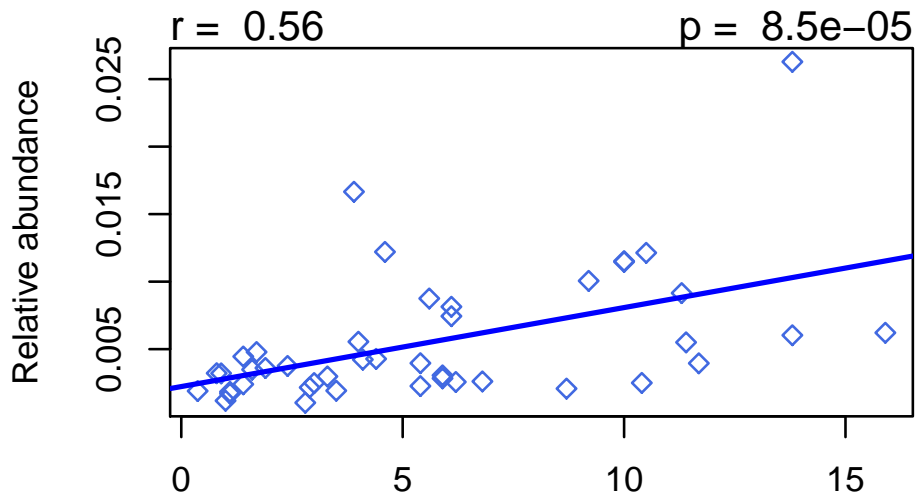

Age (years) 


\section{IgG1_H4N4F1}

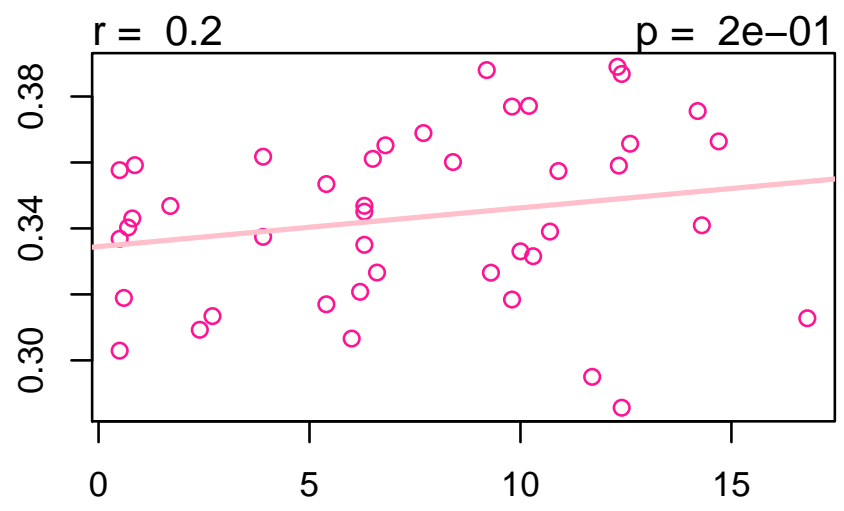

Age (years)

IgG1_H5N4

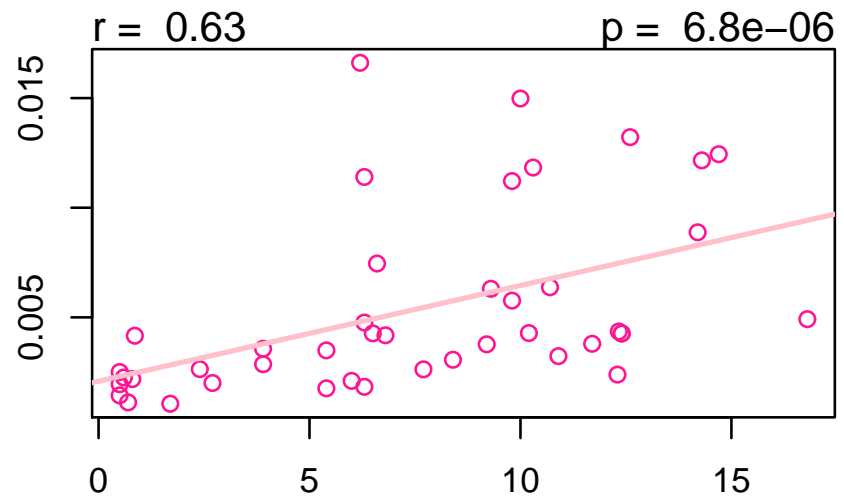

Age (years)

\section{IgG1_H4N4F1}

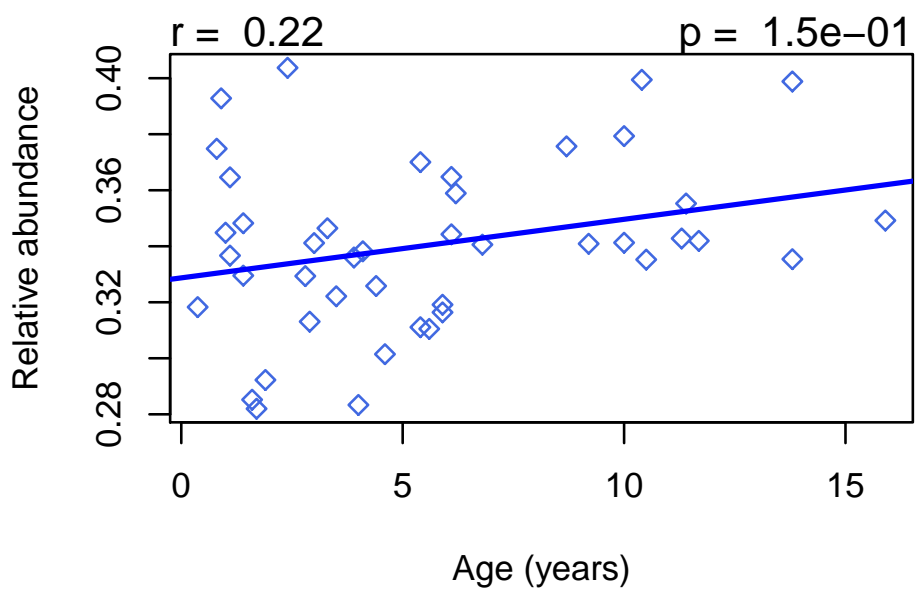

\section{IgG1_H5N4}

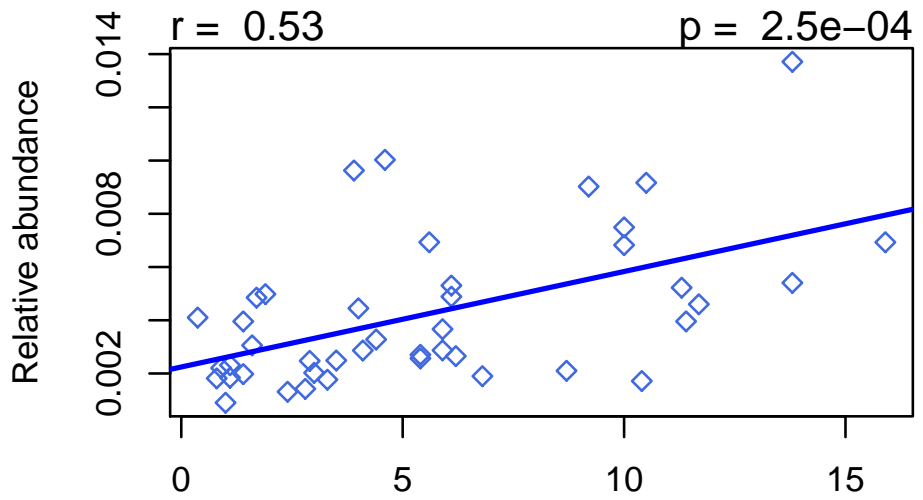

Age (years) 


\section{IgG1_H3N5F1}

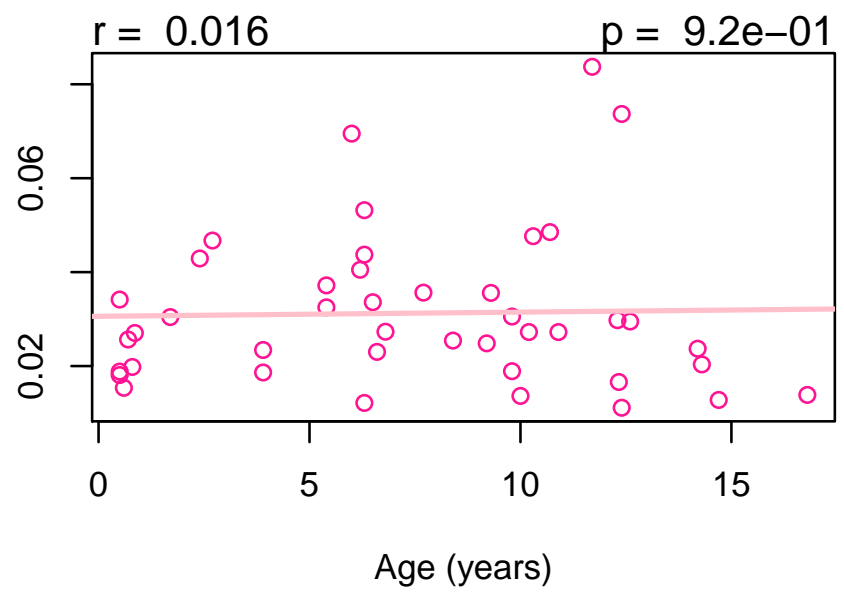

IgG1_H4N5

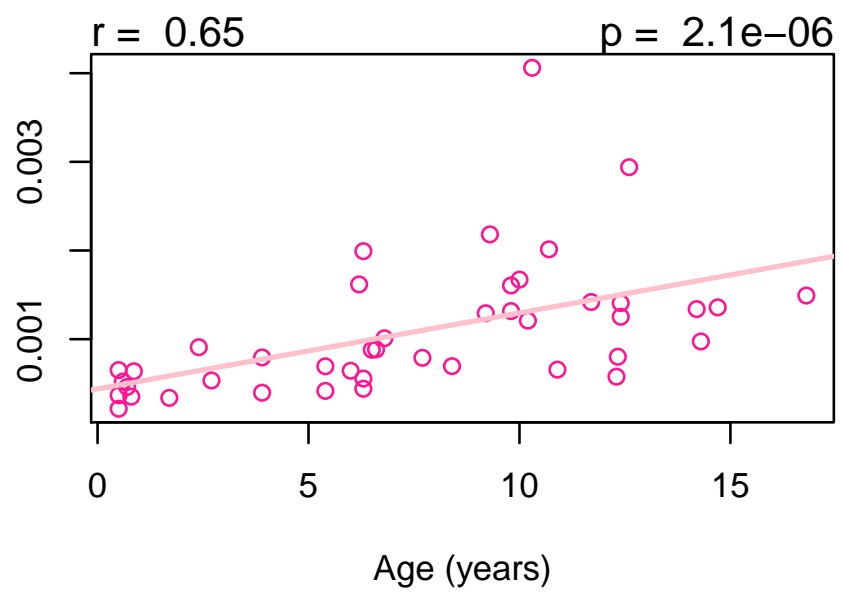

\section{IgG1_H3N5F1}

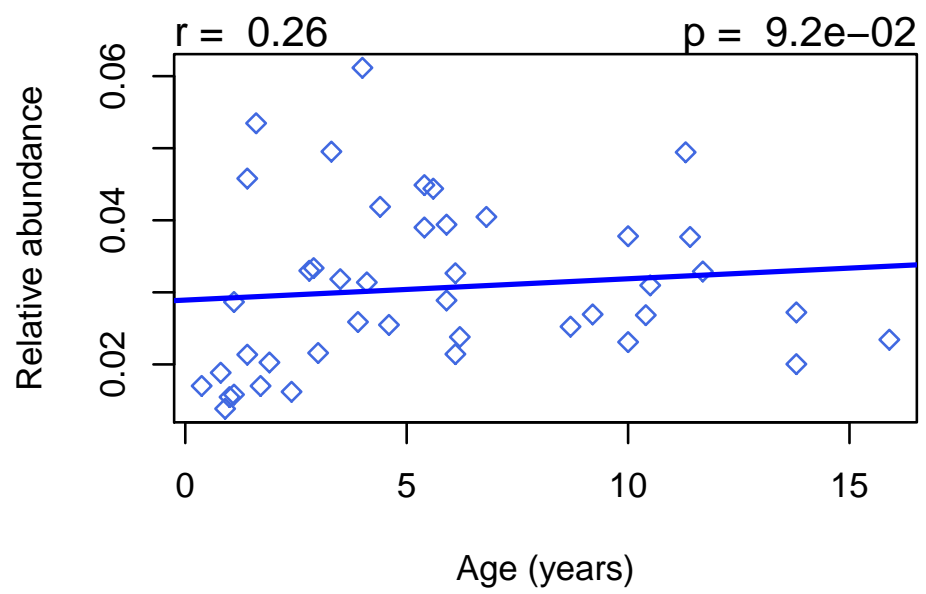

IgG1_H4N5

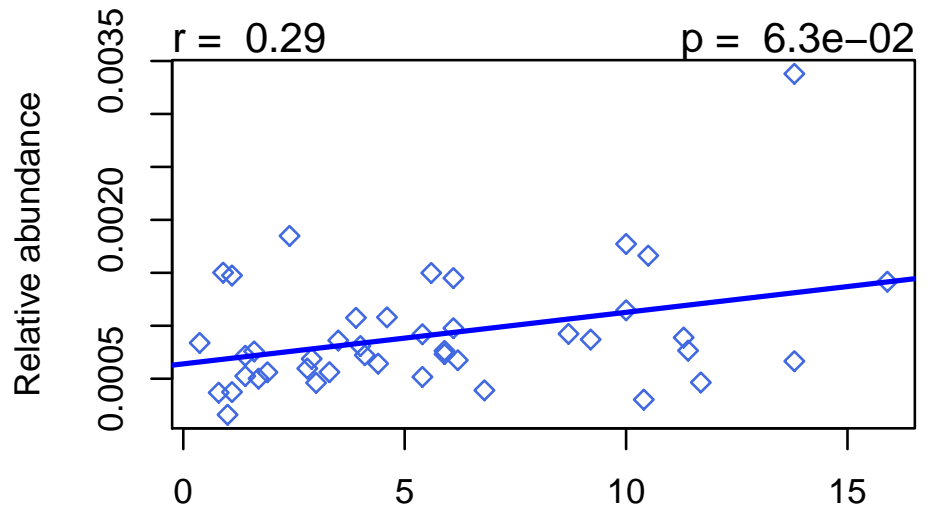

Age (years) 


\section{IgG1_H5N4F1}

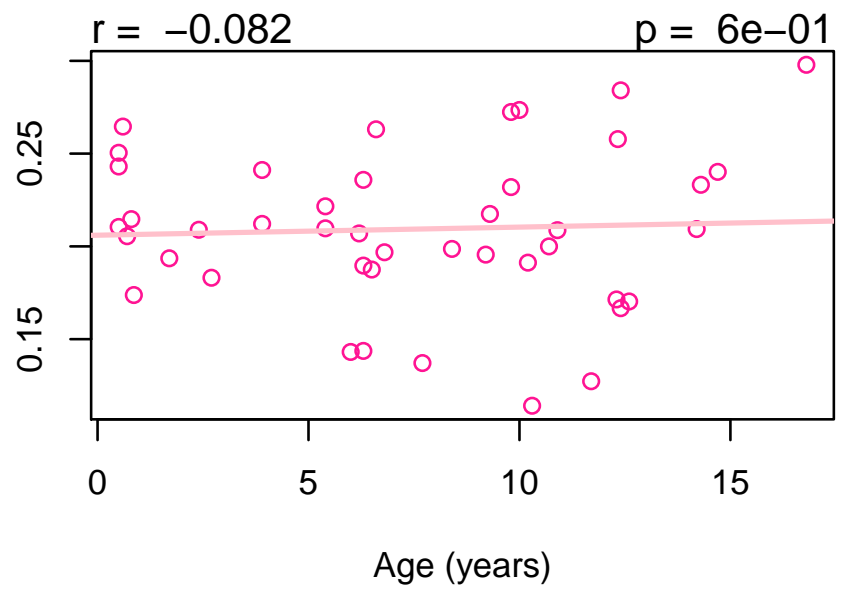

\section{IgG1_H4N5F1}

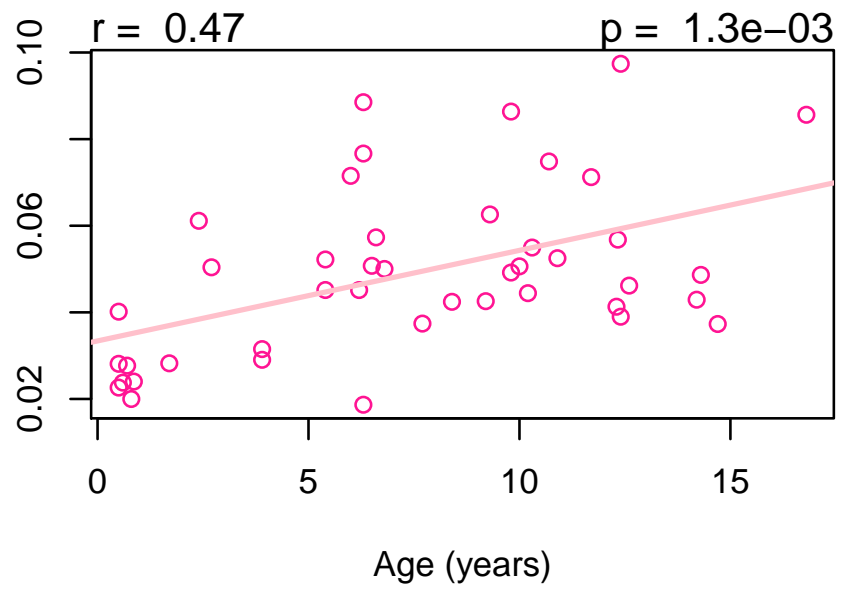

\section{lgG1_H5N4F1}

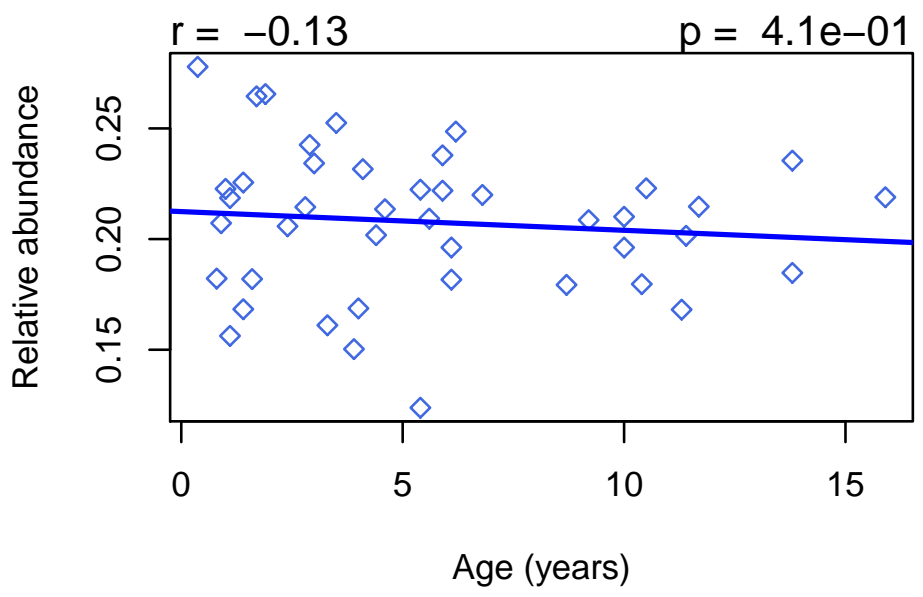

\section{IgG1_H4N5F1}

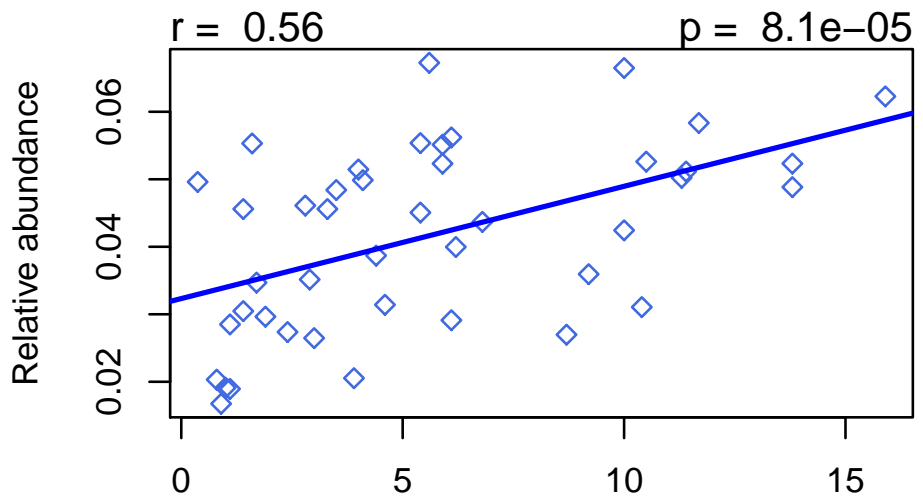

Age (years) 
IgG1_H4N4F1D1

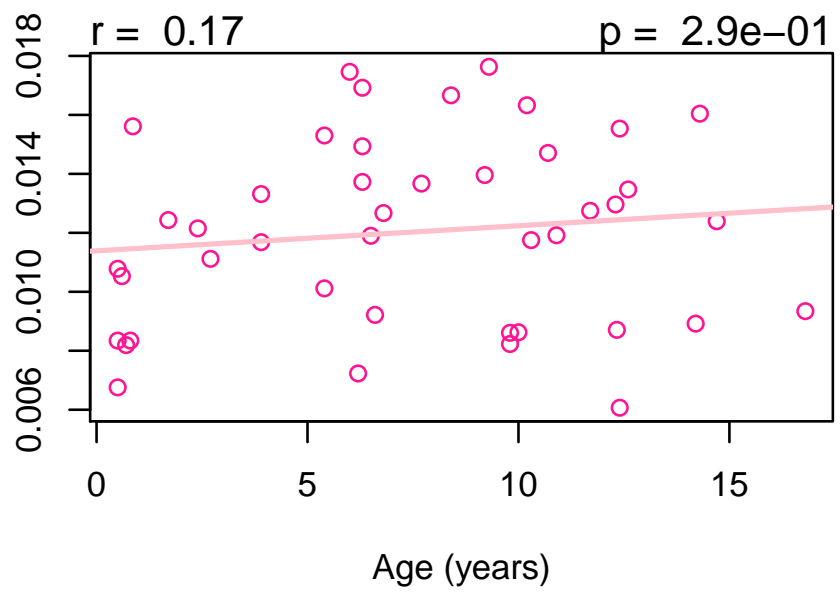

\section{IgG1_H5N4D1}

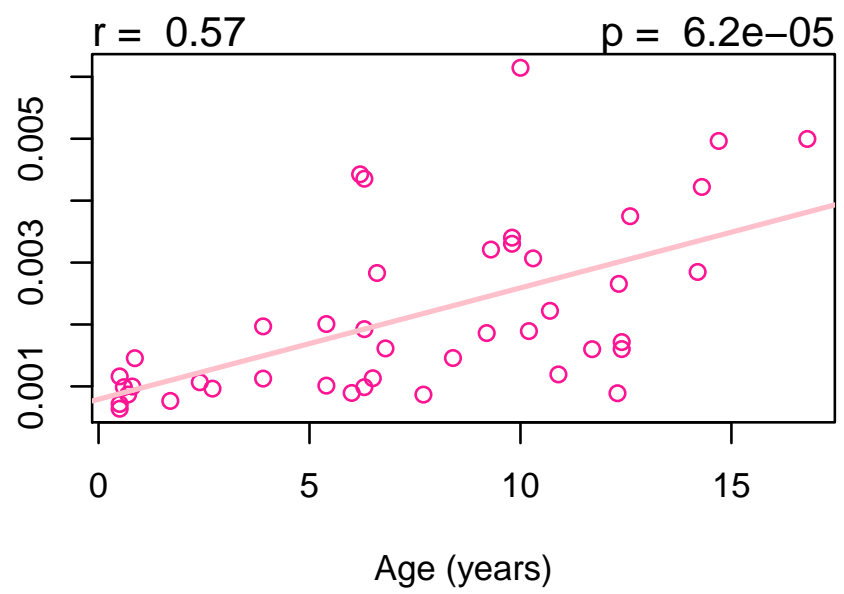

\section{IgG1_H4N4F1D1}

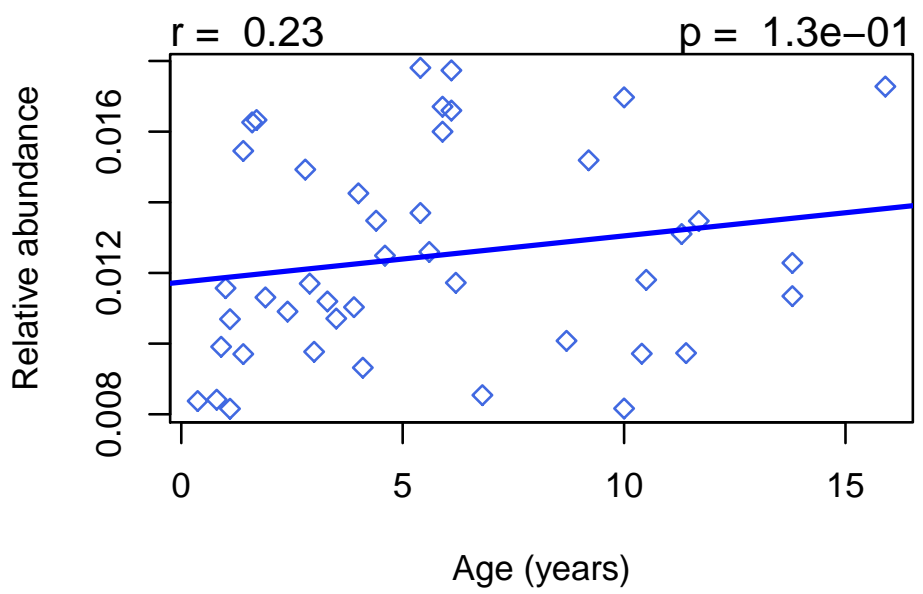

\section{IgG1_H5N4D1}

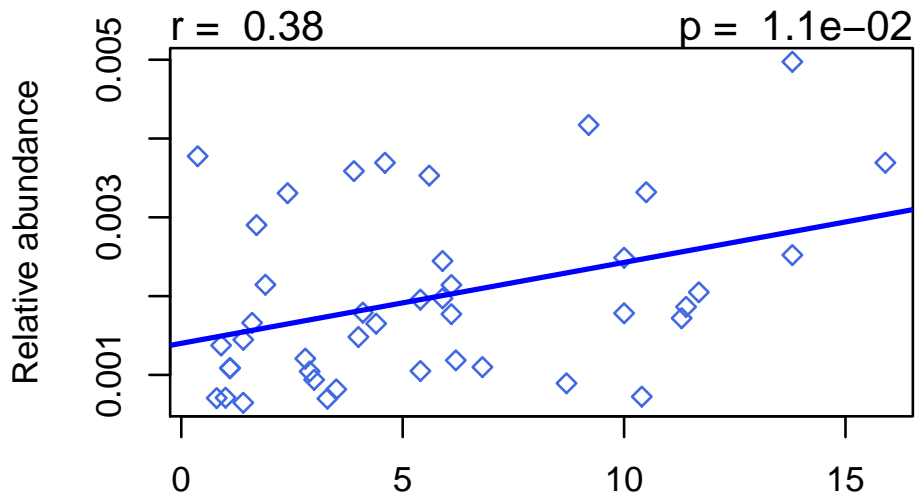

Age (years) 


\section{IgG23_H5N4F1}

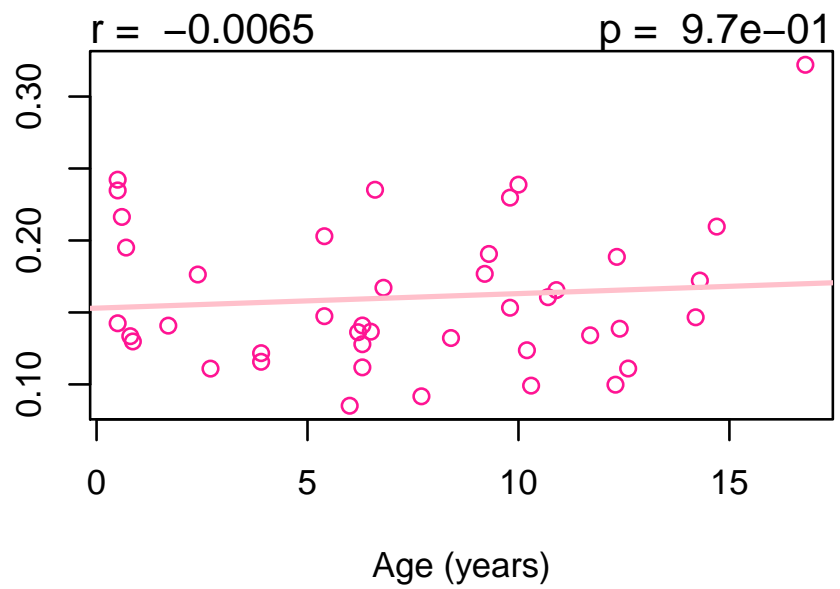

IgG23_H4N4F1D1

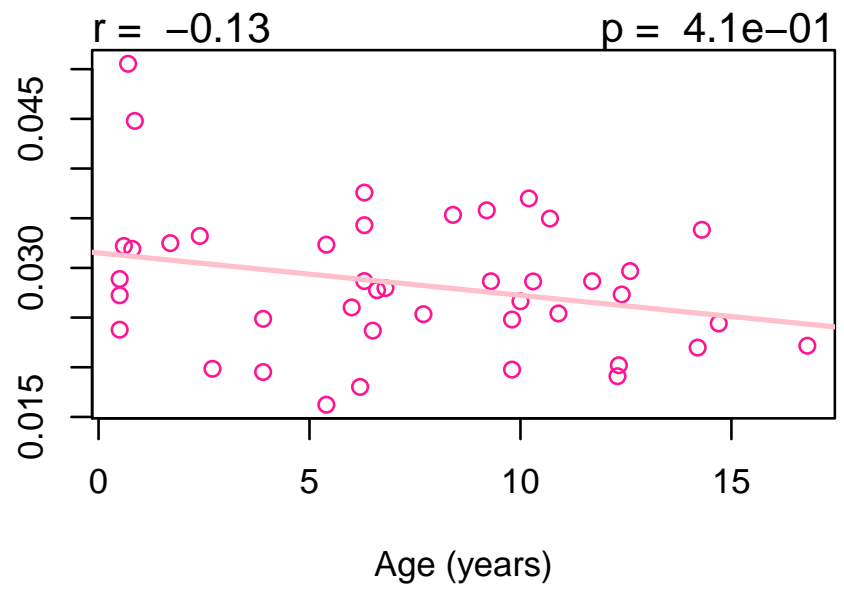

\section{IgG23_H5N4F1}

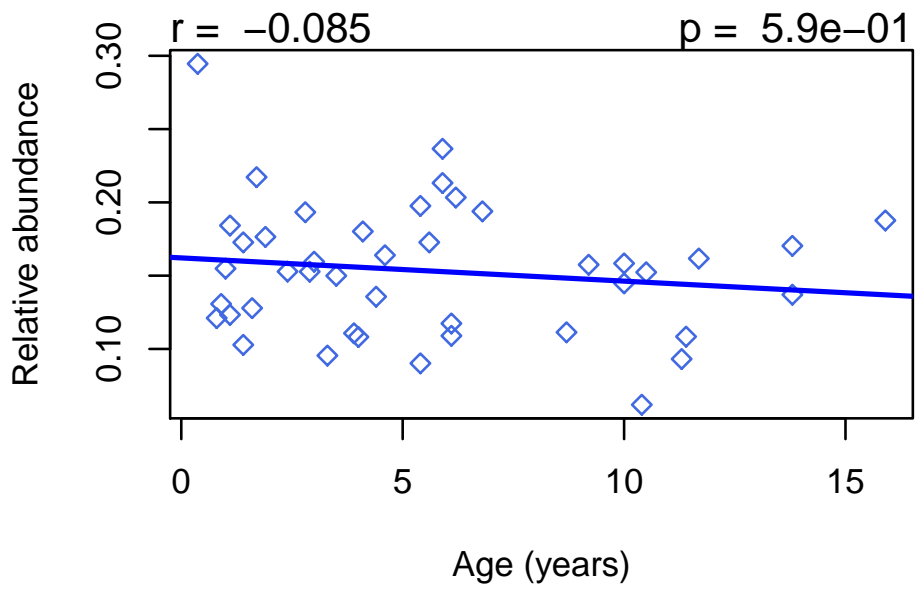

\section{IgG23_H4N4F1D1}

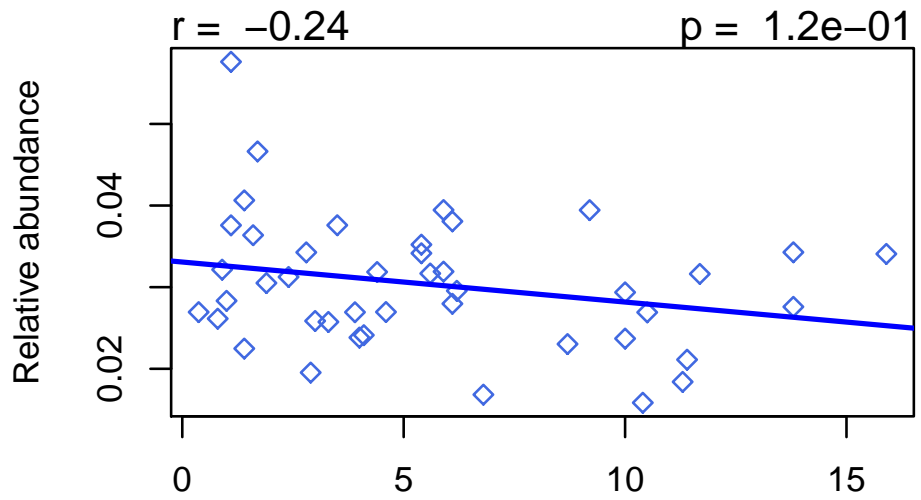

Age (years) 


\section{IgG1_A2}

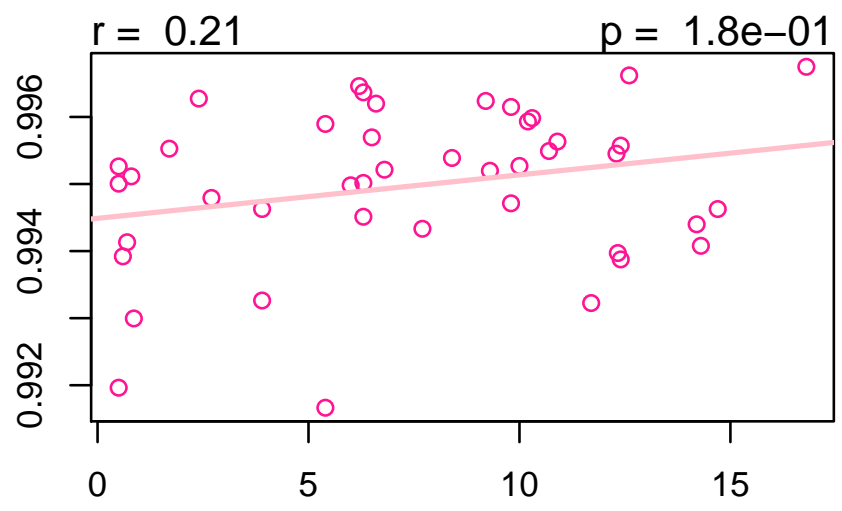

Age (years)

\section{IgG1_B}

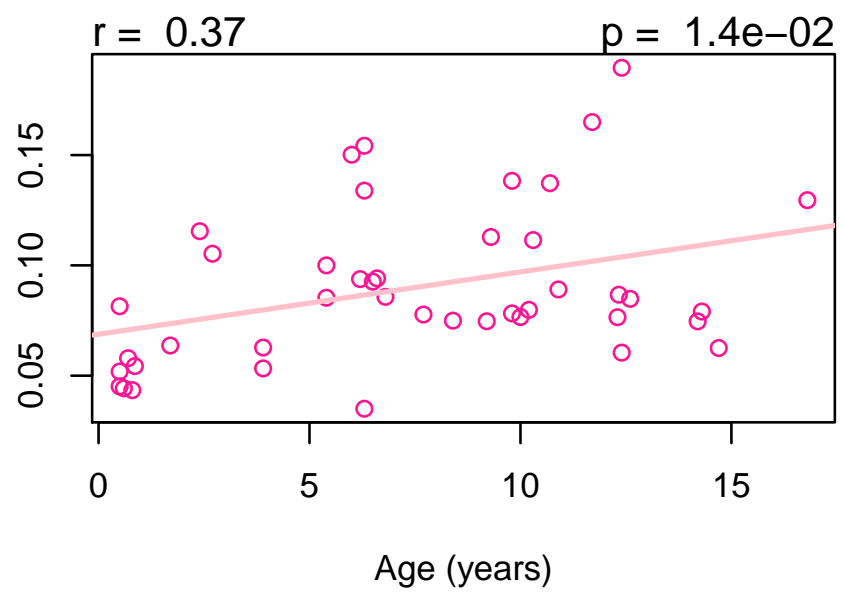

\section{IgG1_A2}

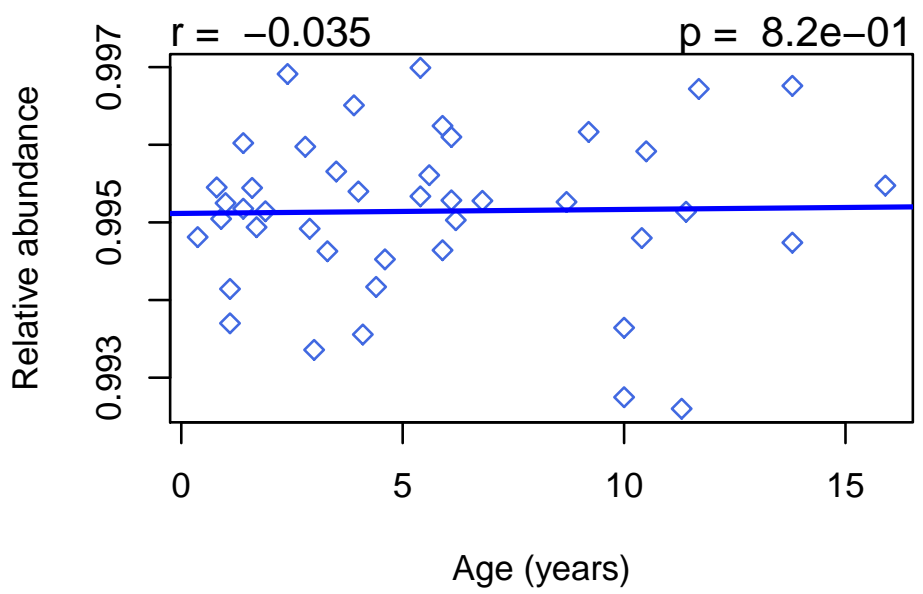

IgG1_B

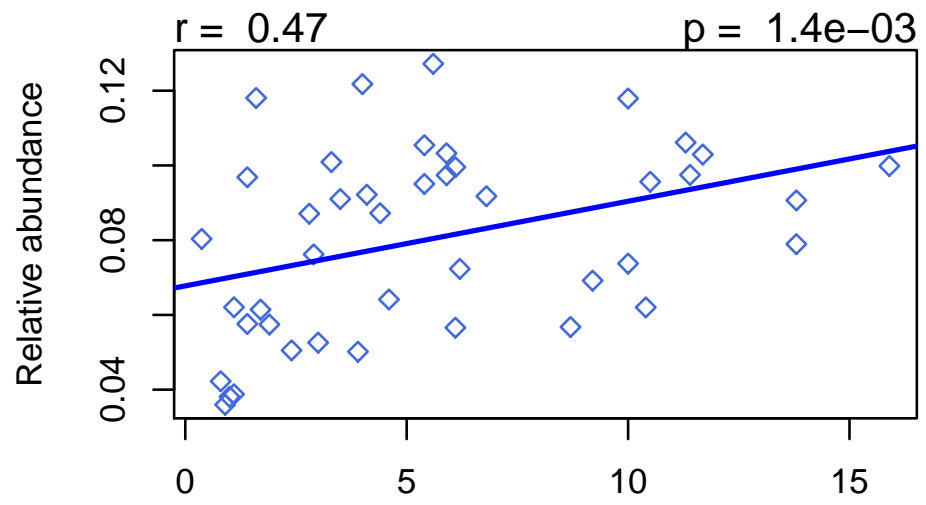

Age (years) 


\section{IgG1_F}

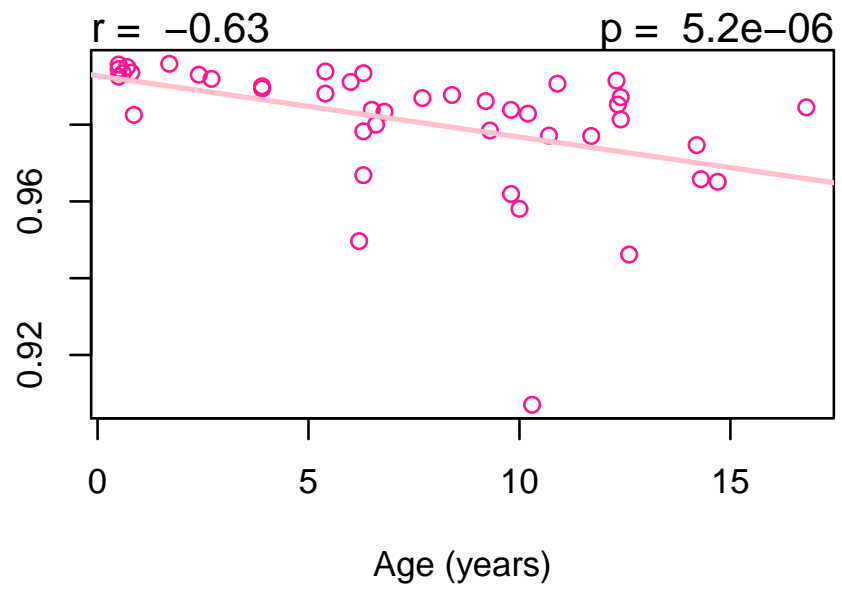

IgG1_A2F

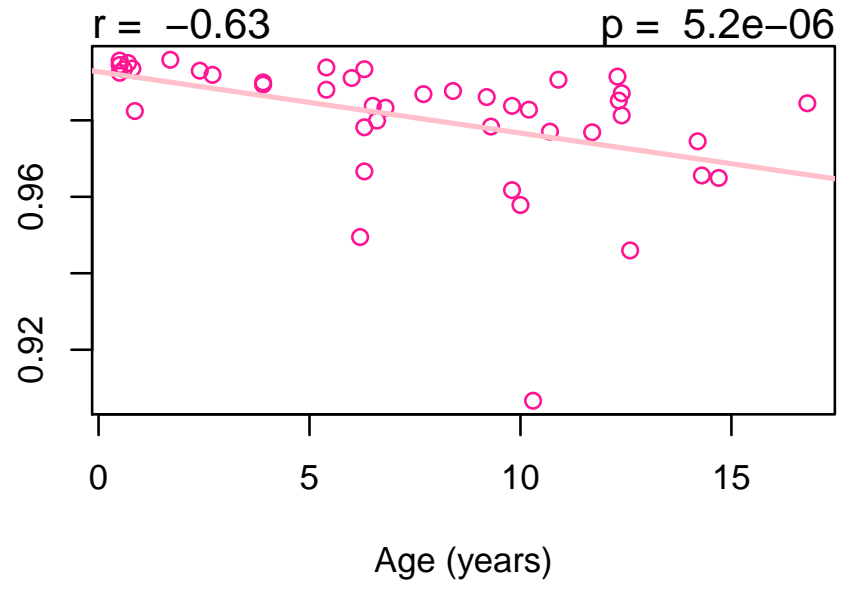

\section{IgG1_F}

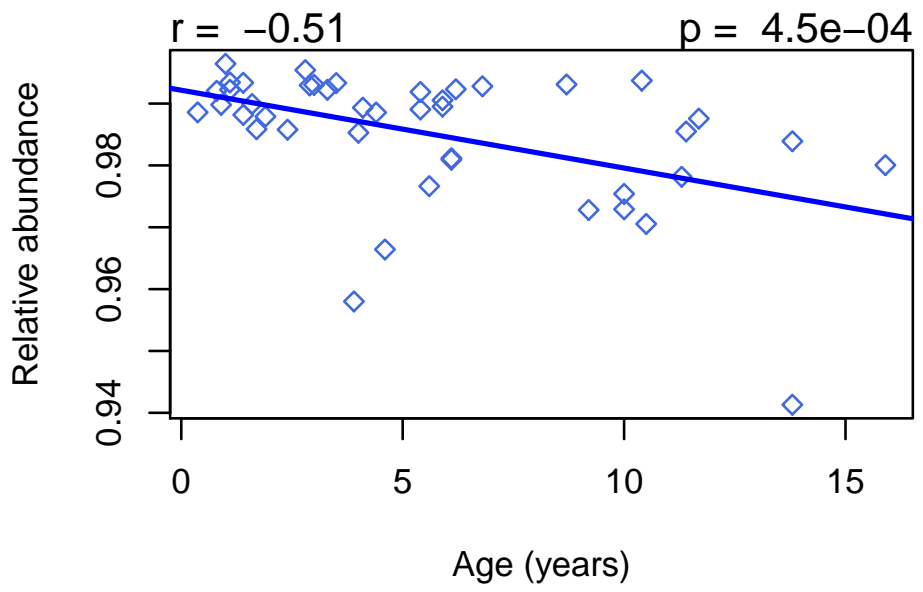

IgG1_A2F

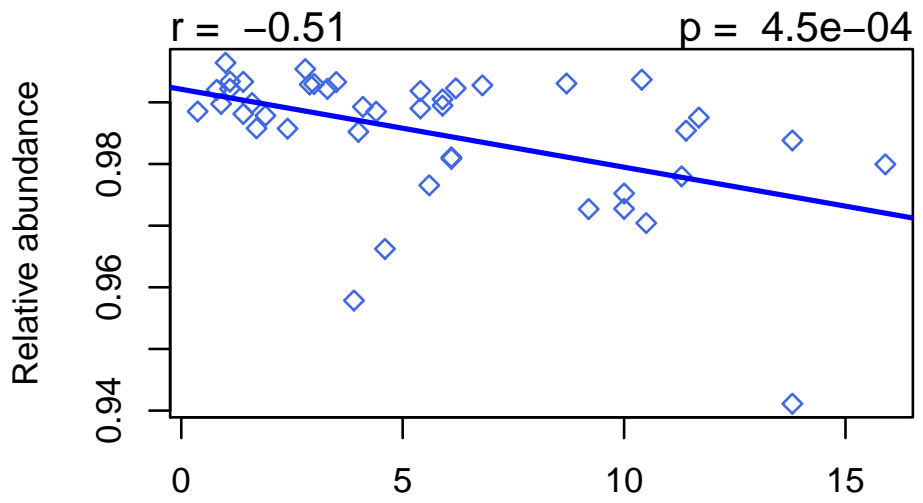

Age (years) 
IgG1_A2B

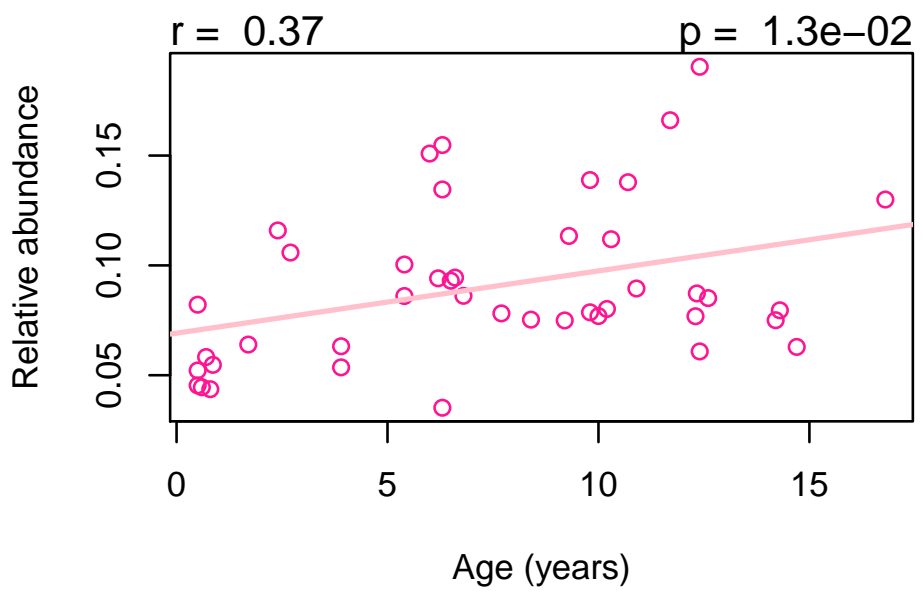

IgG1_A2F0B

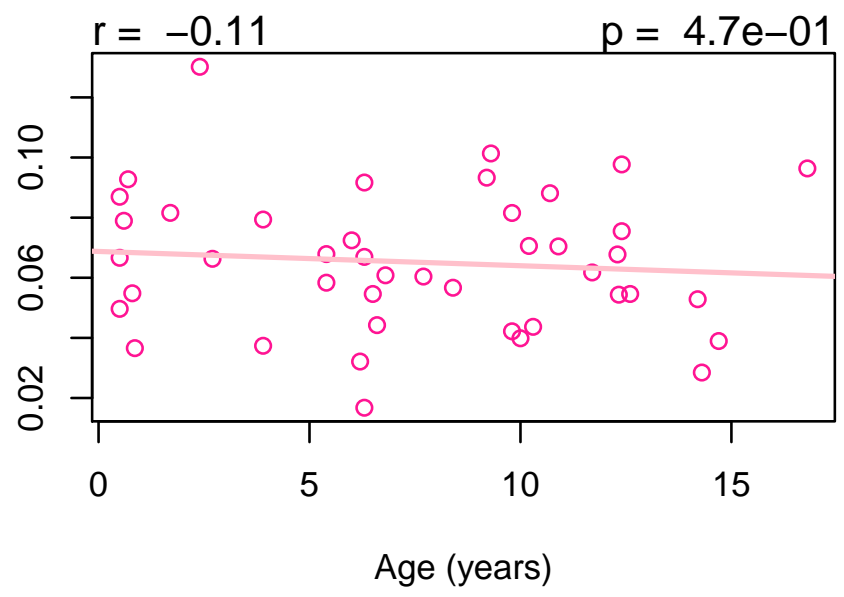

IgG1_A2B

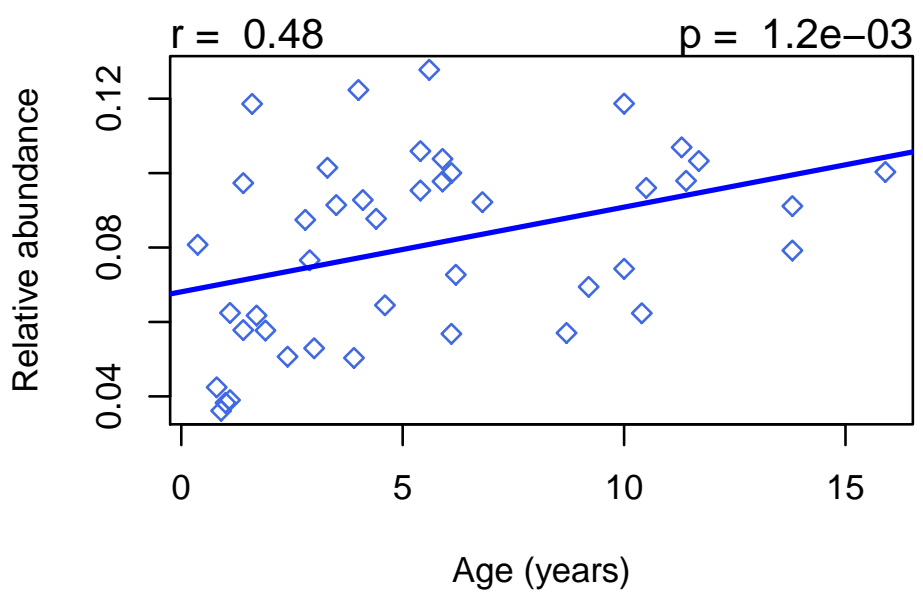

IgG1_A2F0B

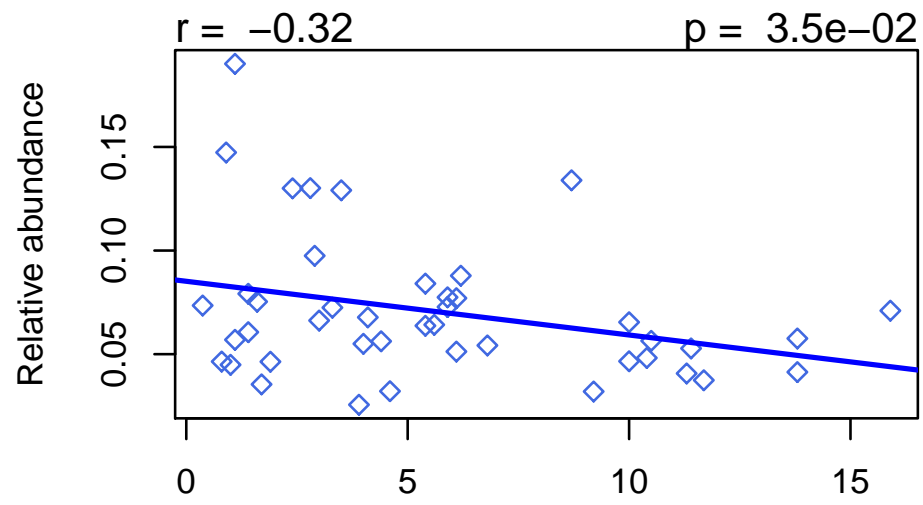

Age (years) 
IgG1_A2D

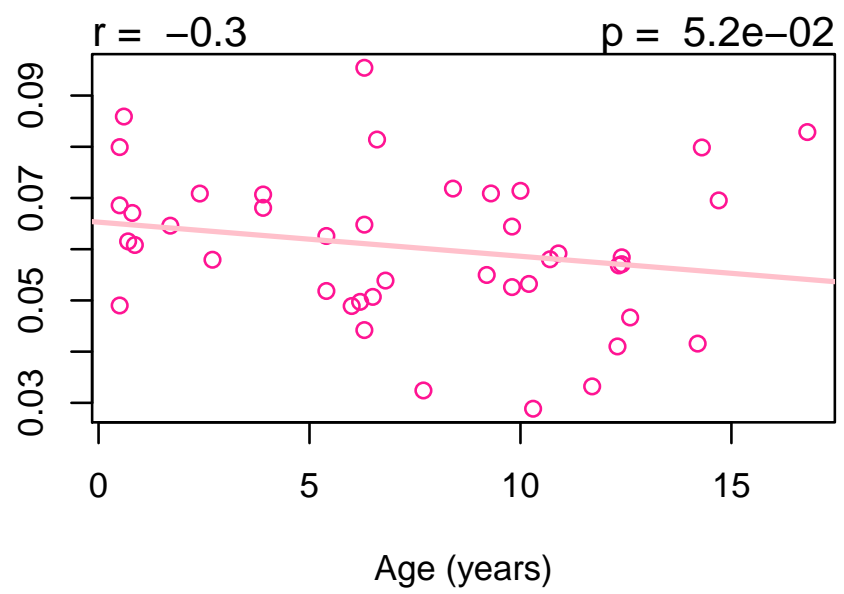

IgG1_A2F0D

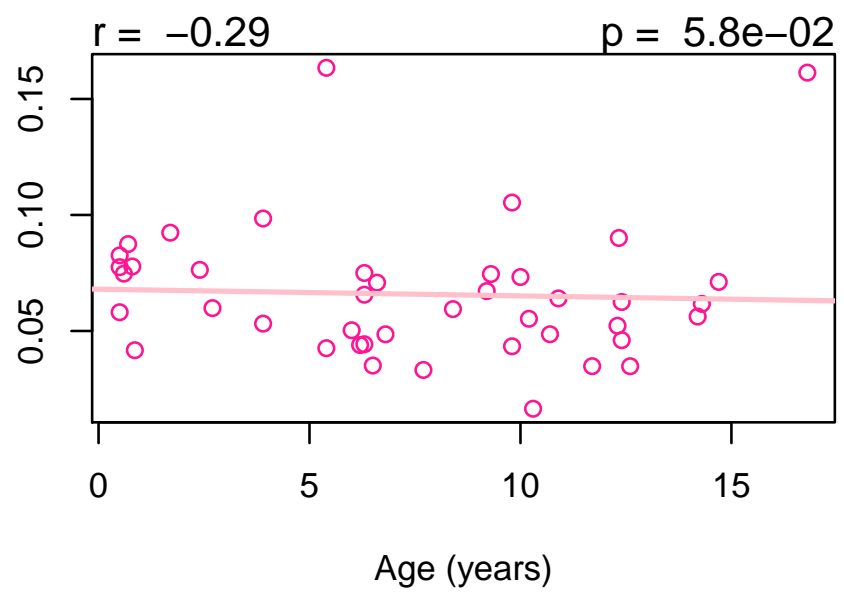

IgG1_A2D

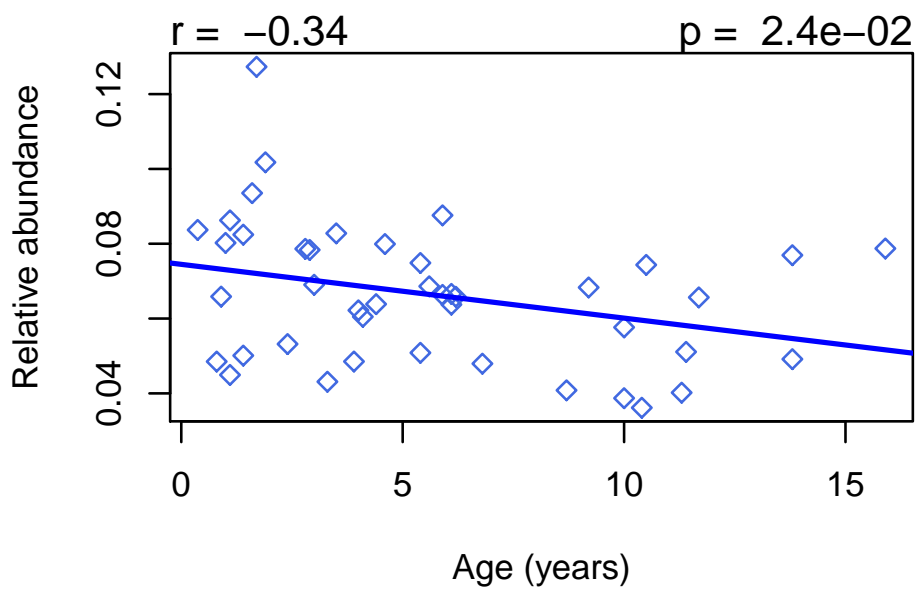

IgG1_A2F0D

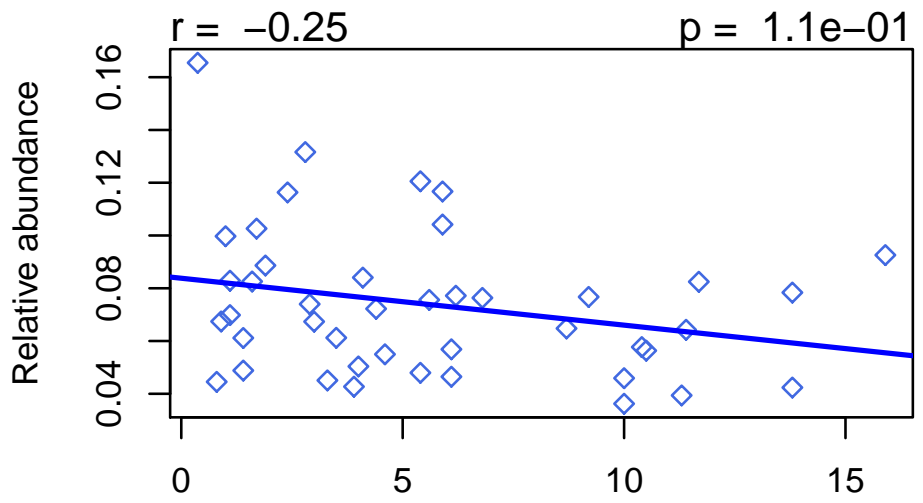

Age (years) 


\section{IgG1_A2FD}

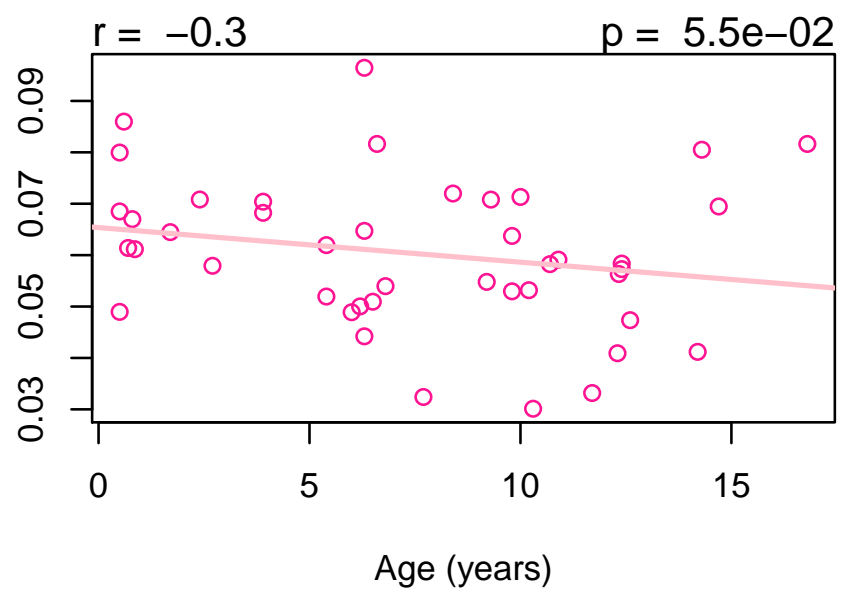

\section{IgG1_A2GD}

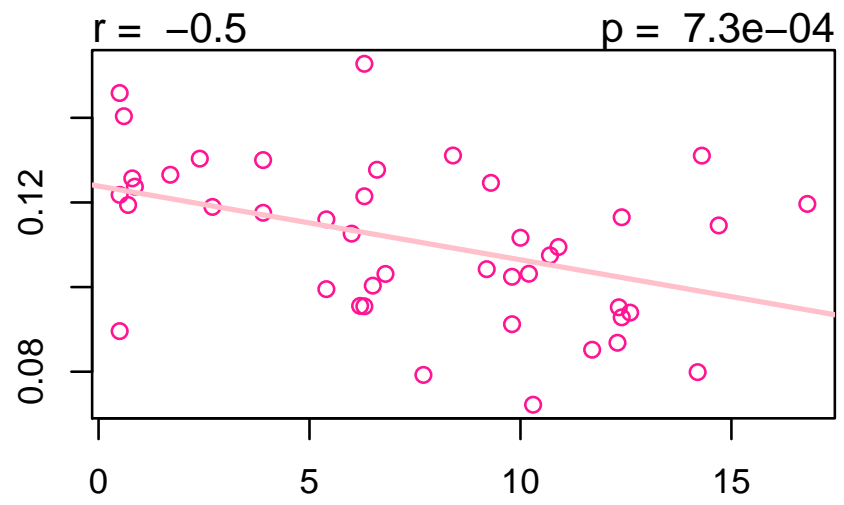

Age (years)

\section{IgG1_A2FD}

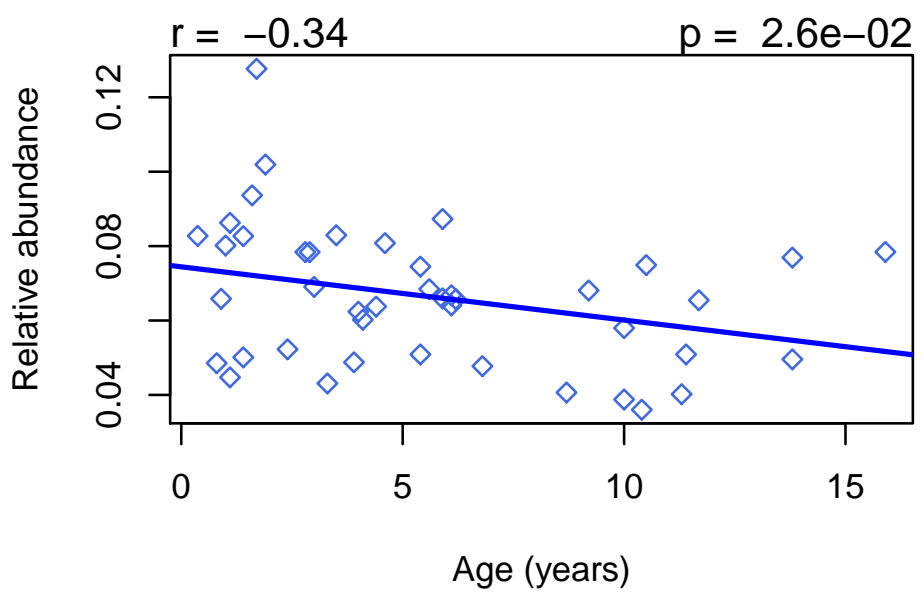

\section{IgG1_A2GD}

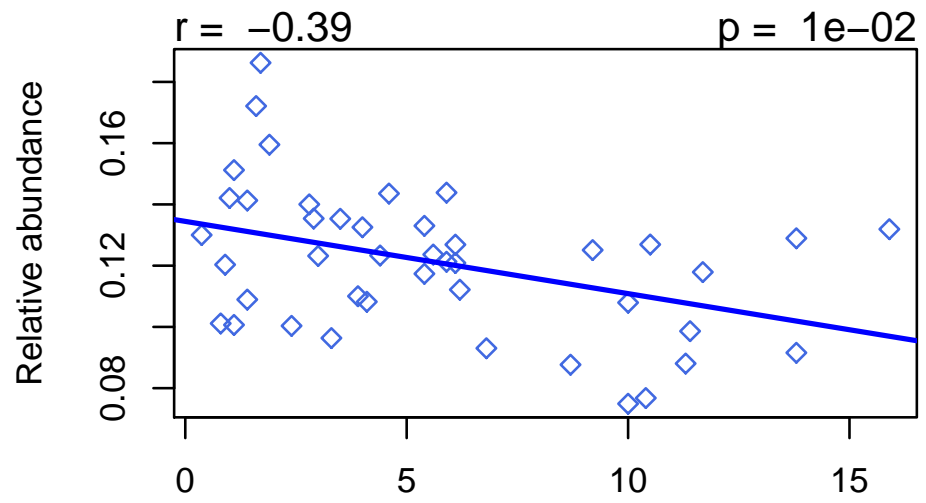

Age (years) 
IgG1_A2F0GD

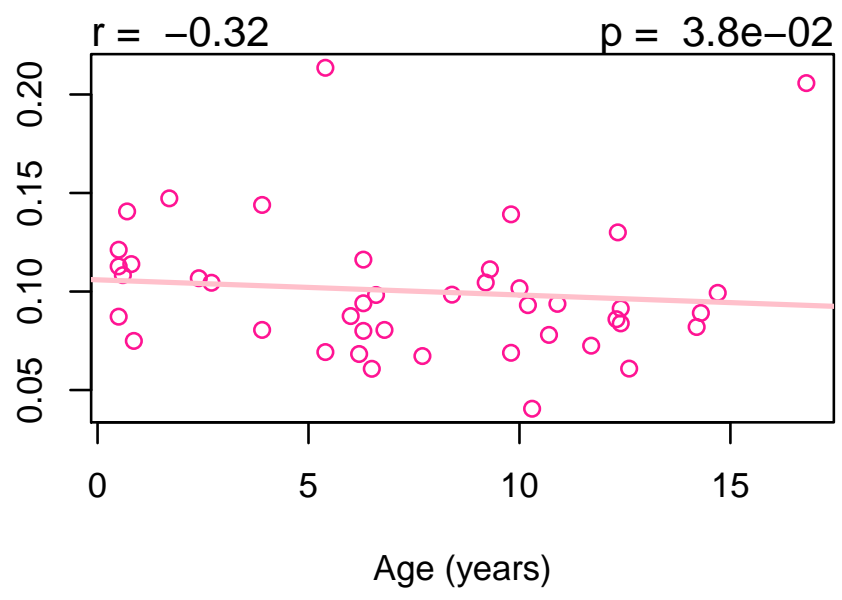

IgG1_A2FGD

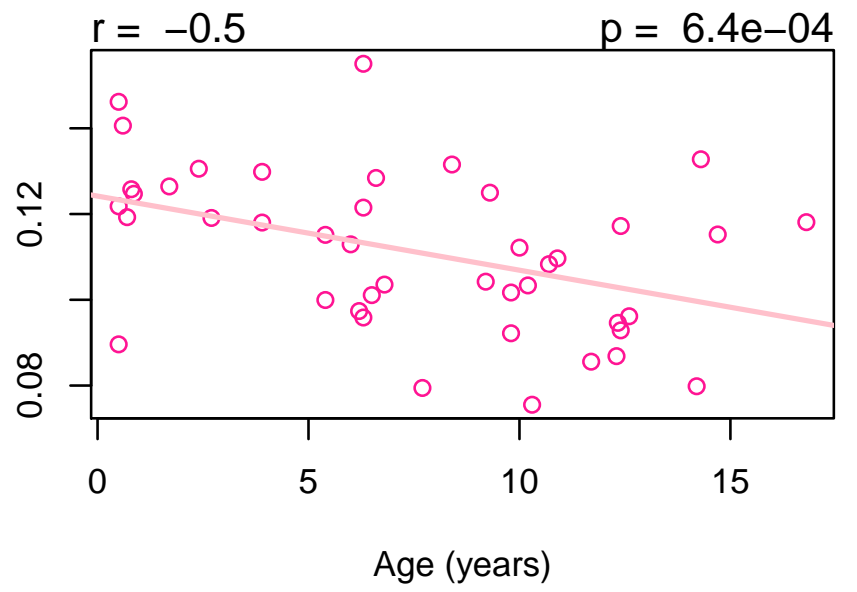

IgG1_A2F0GD

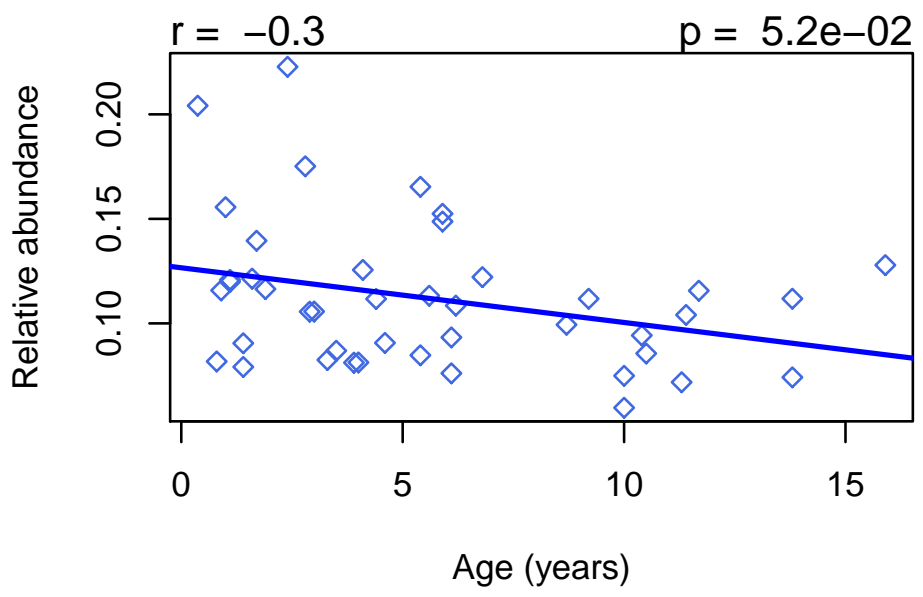

\section{IgG1_A2FGD}

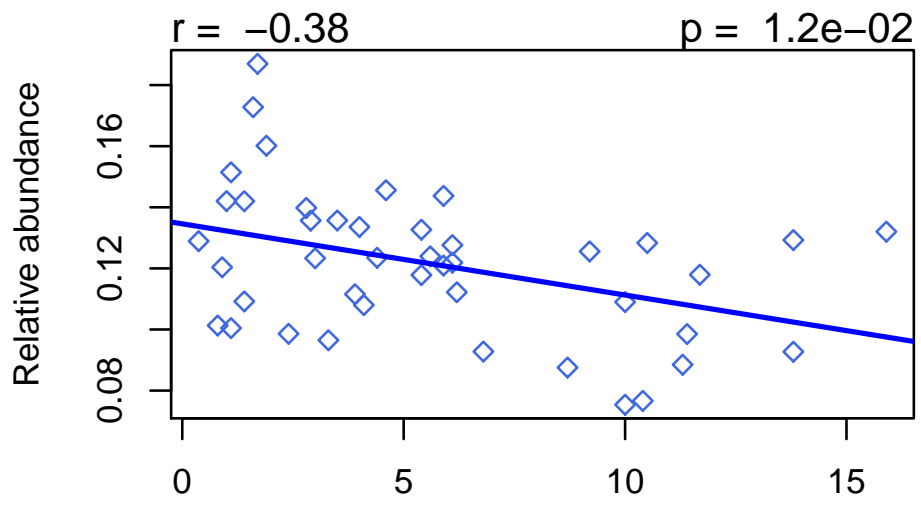


IgG1_A2FD_B

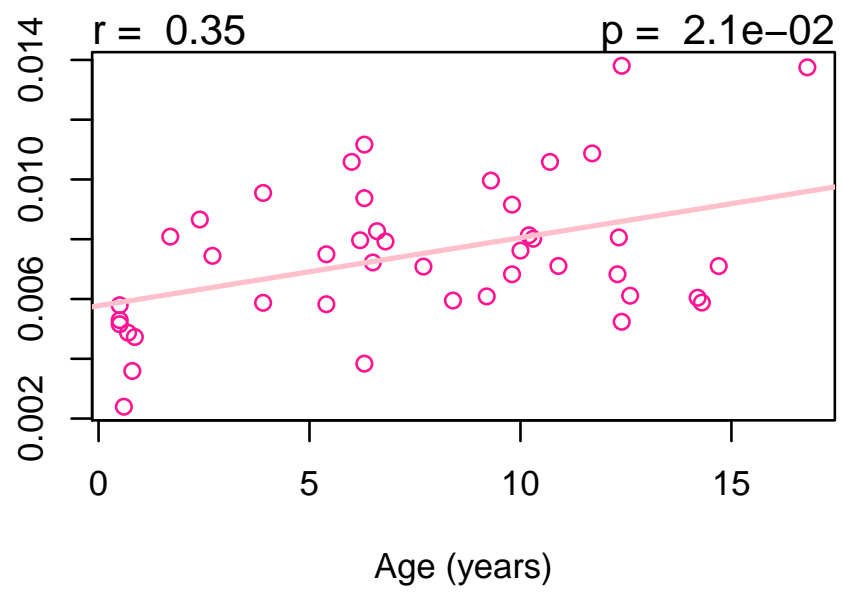

IgG1_agal

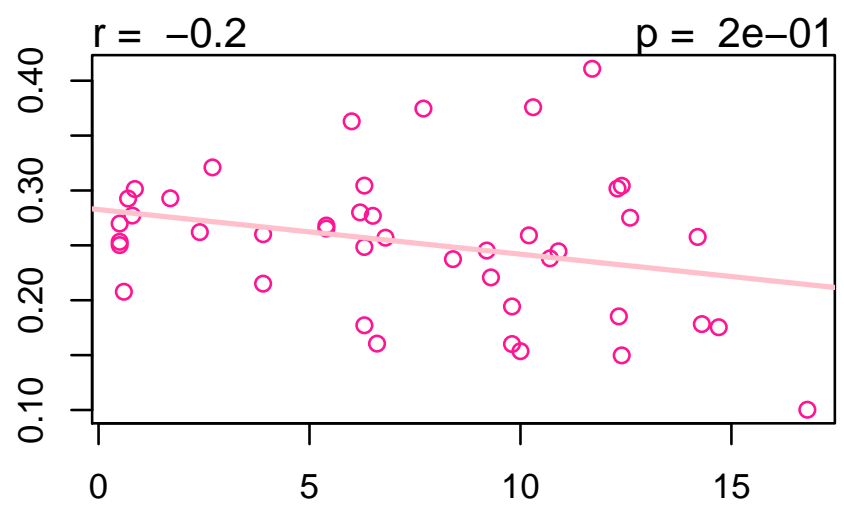

Age (years)
IgG1_A2FD_B

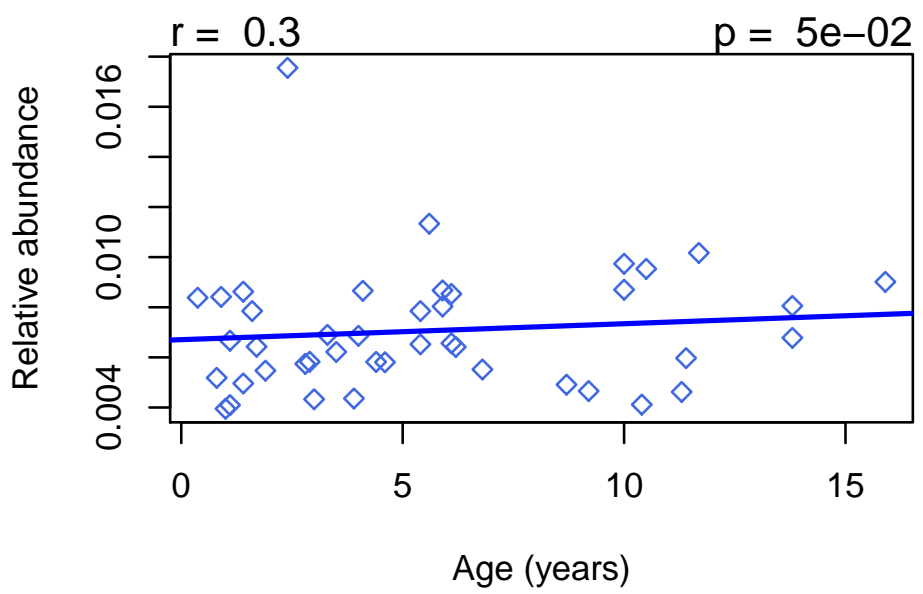

IgG1_agal

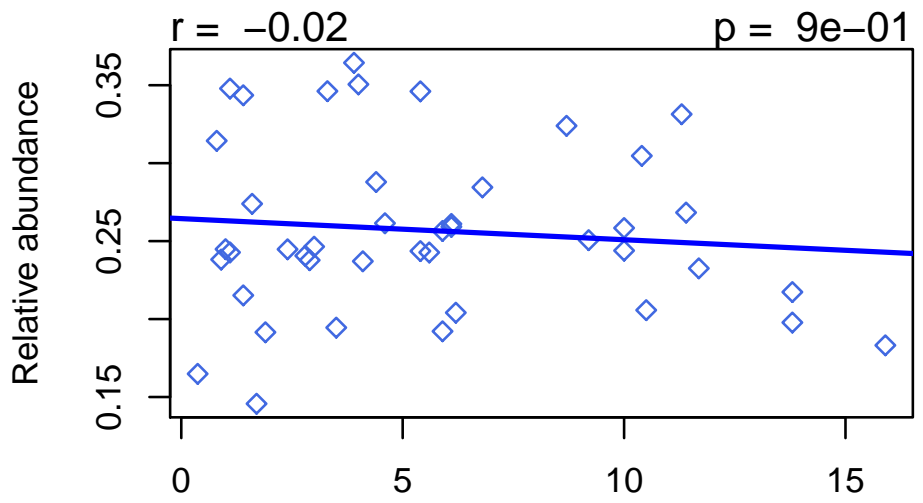

Age (years) 


\section{IgG23_A2FG}

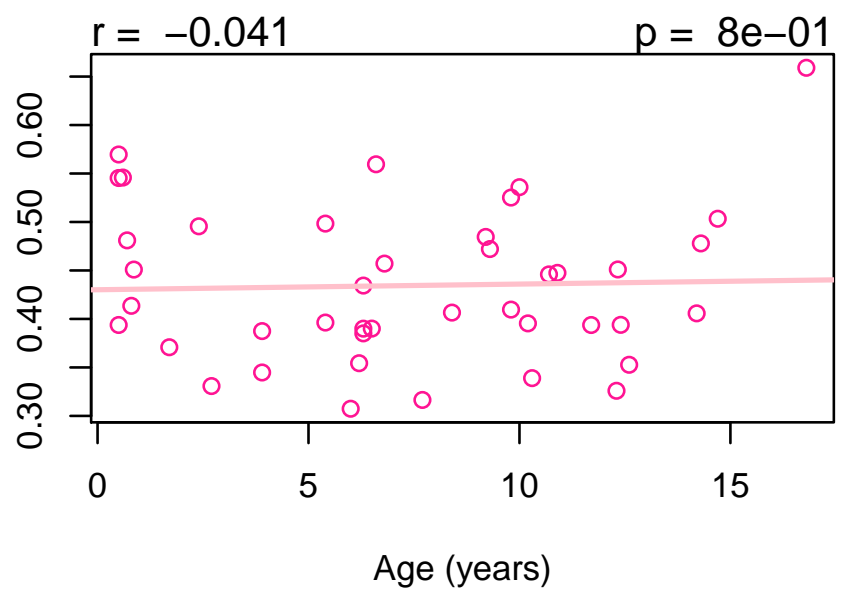

IgG23_A2FD

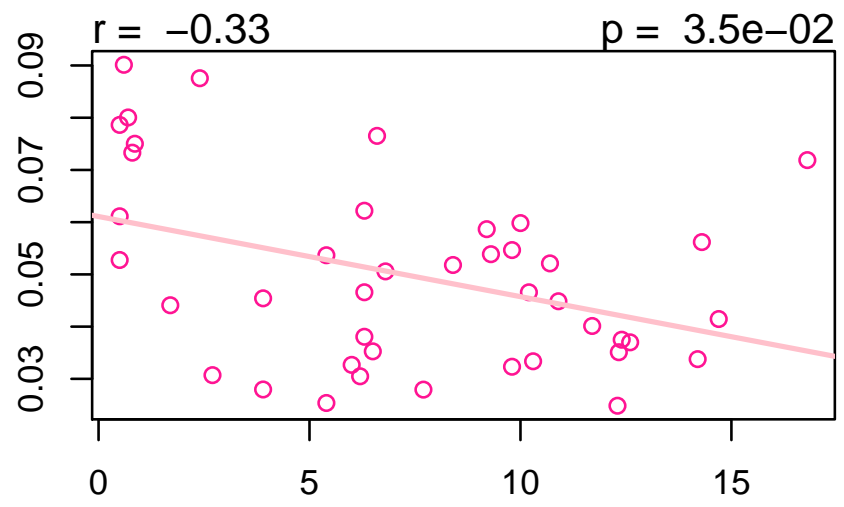

Age (years)

\section{IgG23_A2FG}

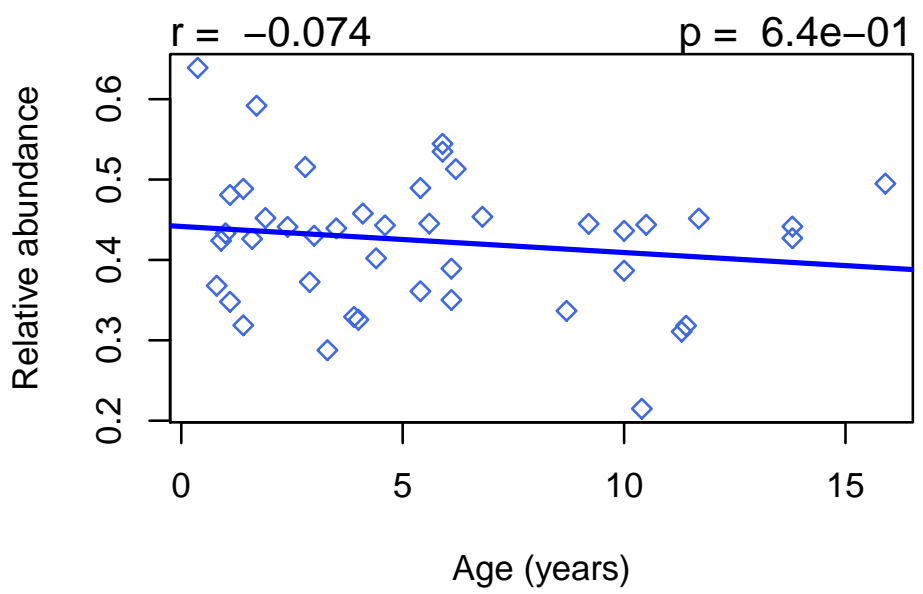

\section{IgG23_A2FD}

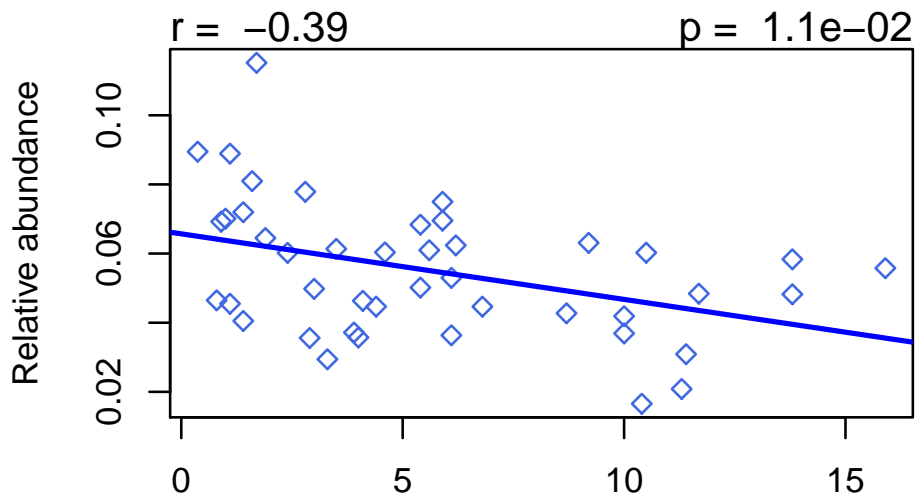

Age (years) 
IgG23_A2FGD

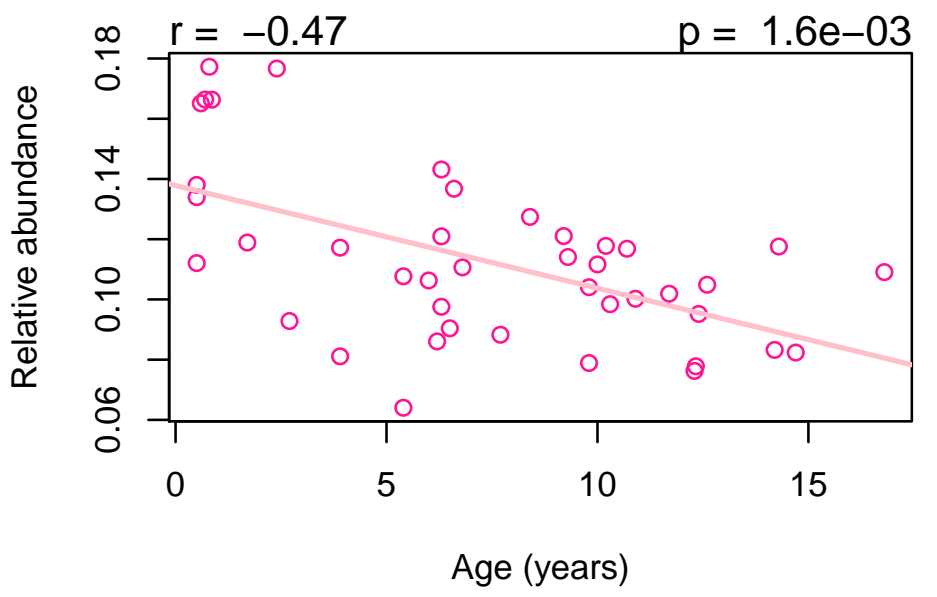

\section{IgG23_A2FGD}

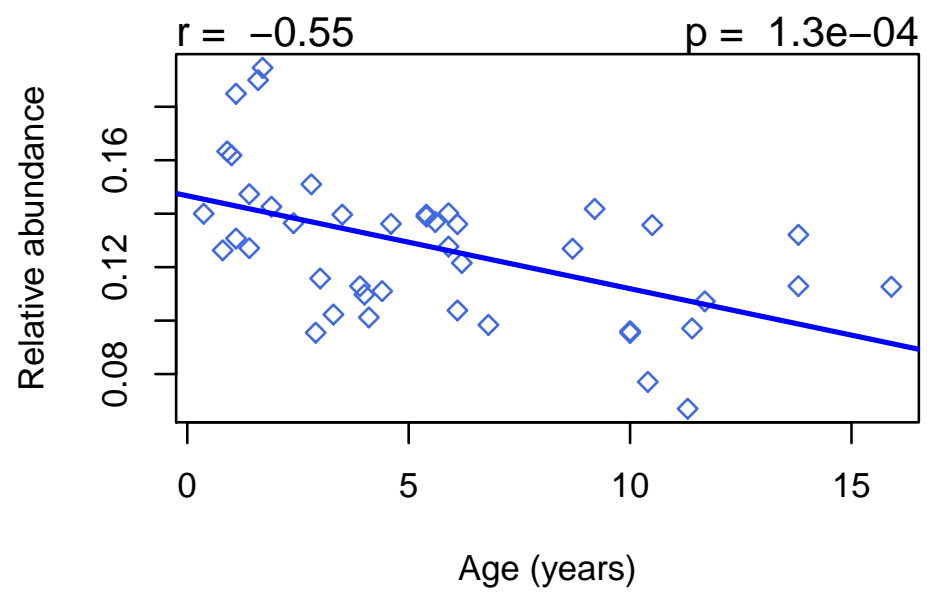


Figure S-3 Levels of all glycans and derived glycan traits of IgG1 and IgG2/3. Pink circles: female, blue diamonds: male, $r$ : correlation coefficient. The linear lines were fitted through combined female and male samples and is not related to the calculated correlation coefficient. After Bonferroni correction, $p$-values below 8.7E-5 were considered statistically significant.

\section{IgG1_H3N3F1}

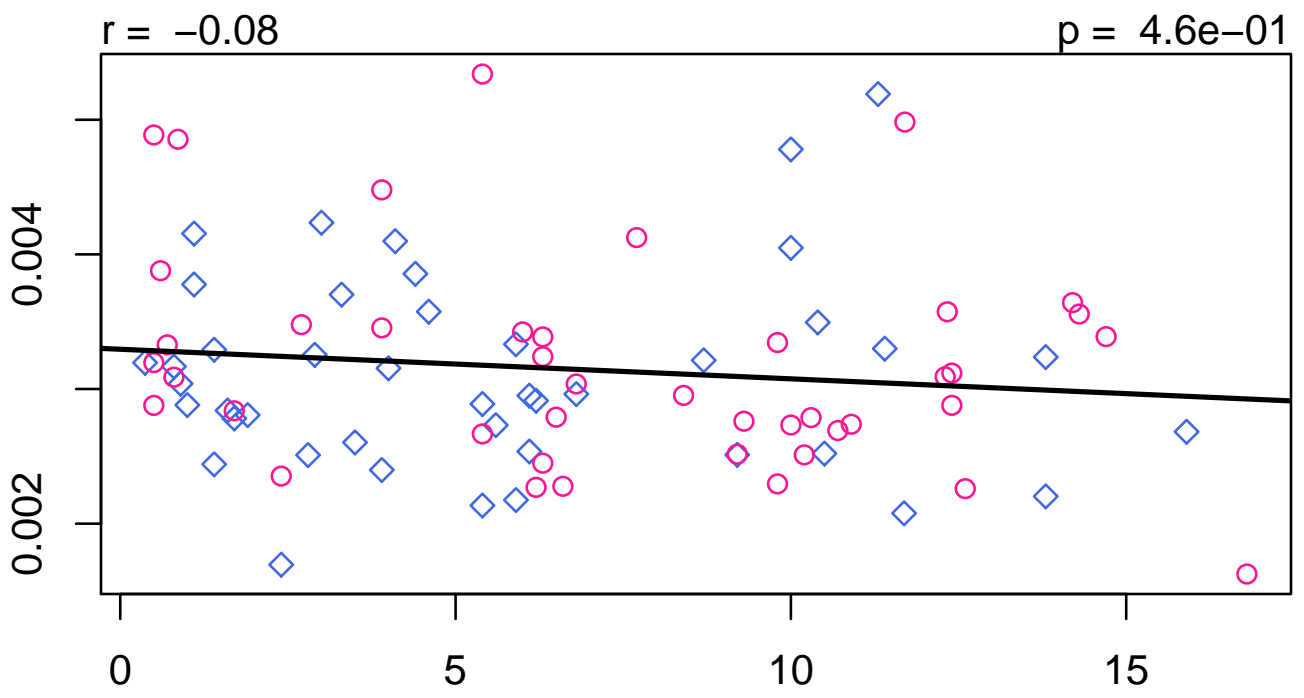




\section{IgG1_H3N4F1}

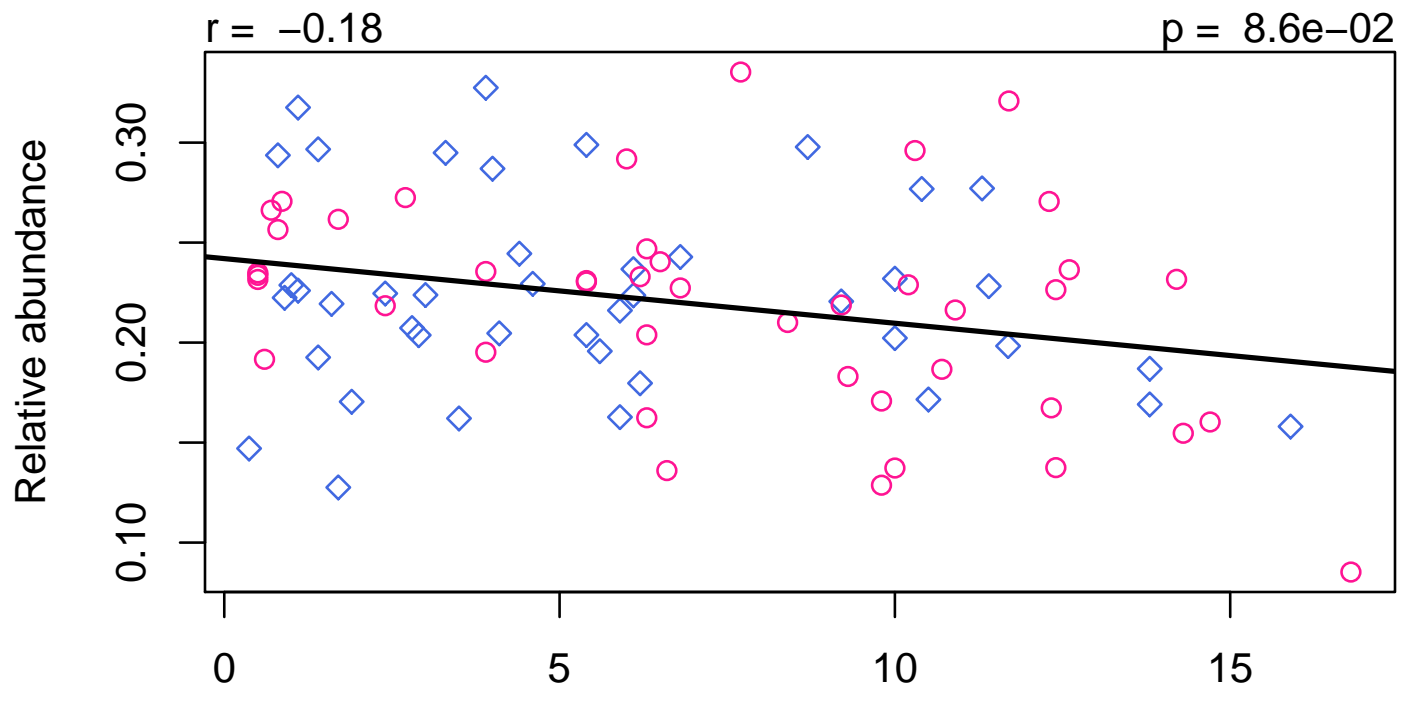

Age (years)

IgG1_H4N4

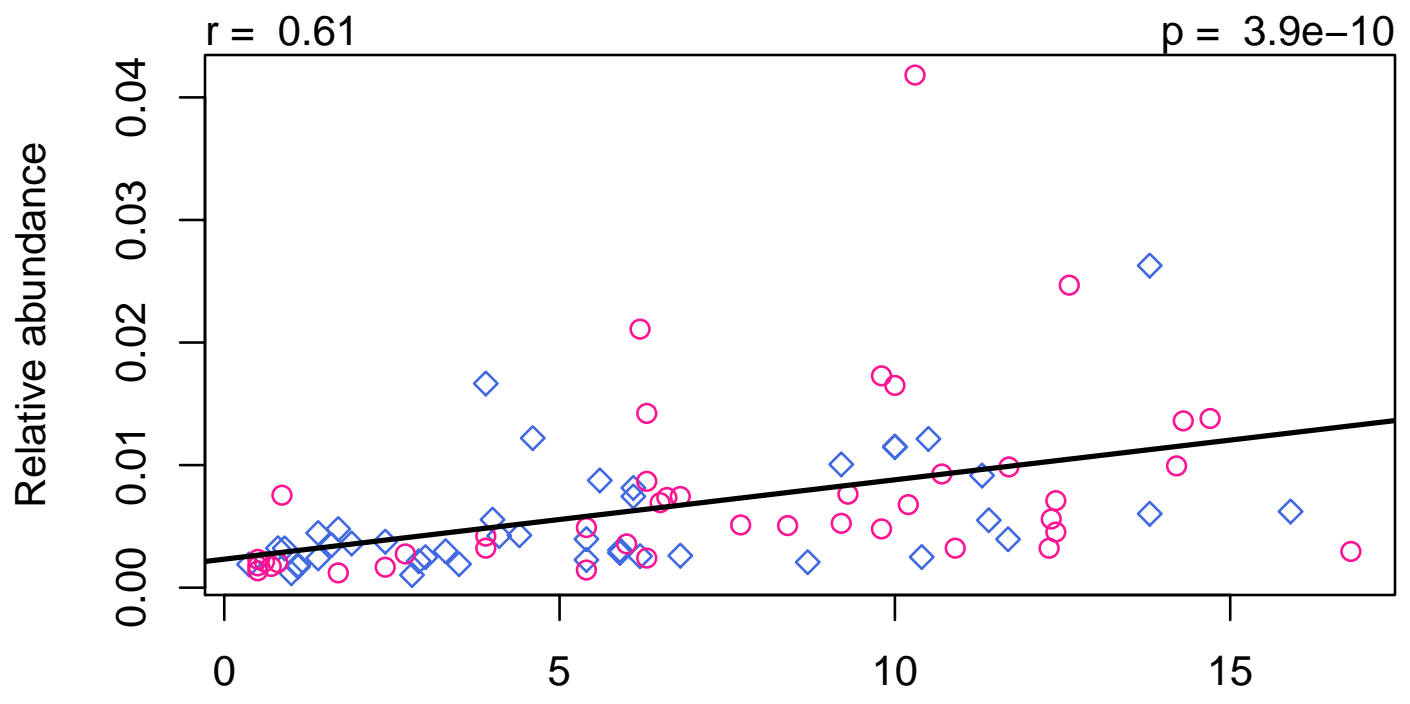


IgG1_H3N5F1

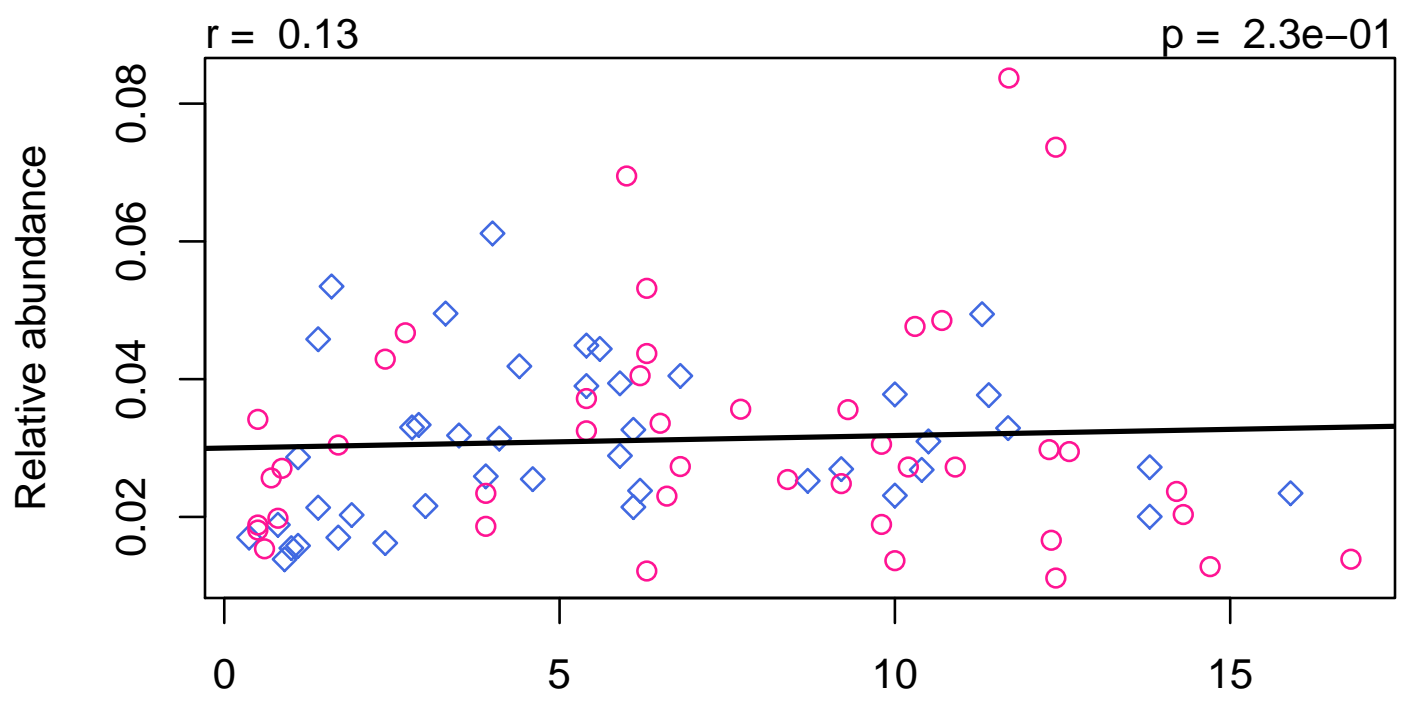

Age (years)

IgG1_H4N5

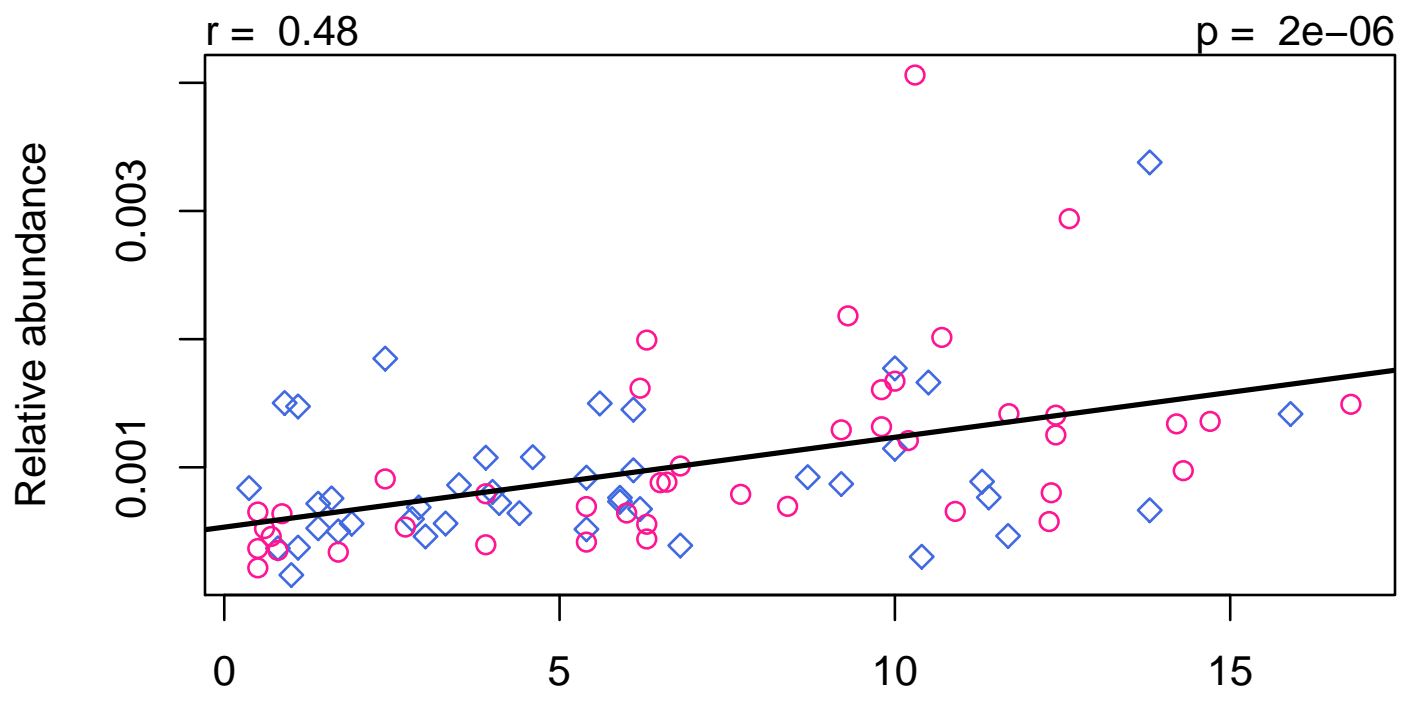

Age (years) 
IgG1_H5N4F1

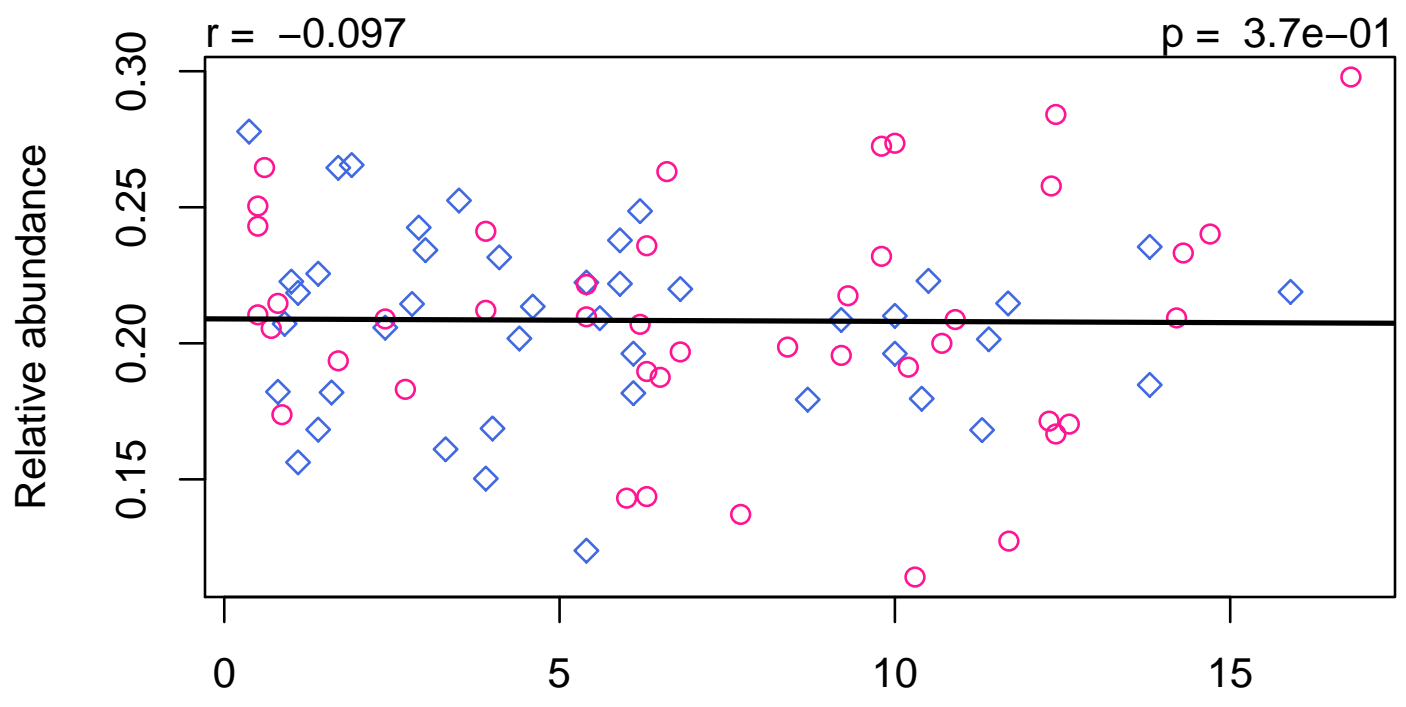

Age (years)

IgG1_H4N5F1

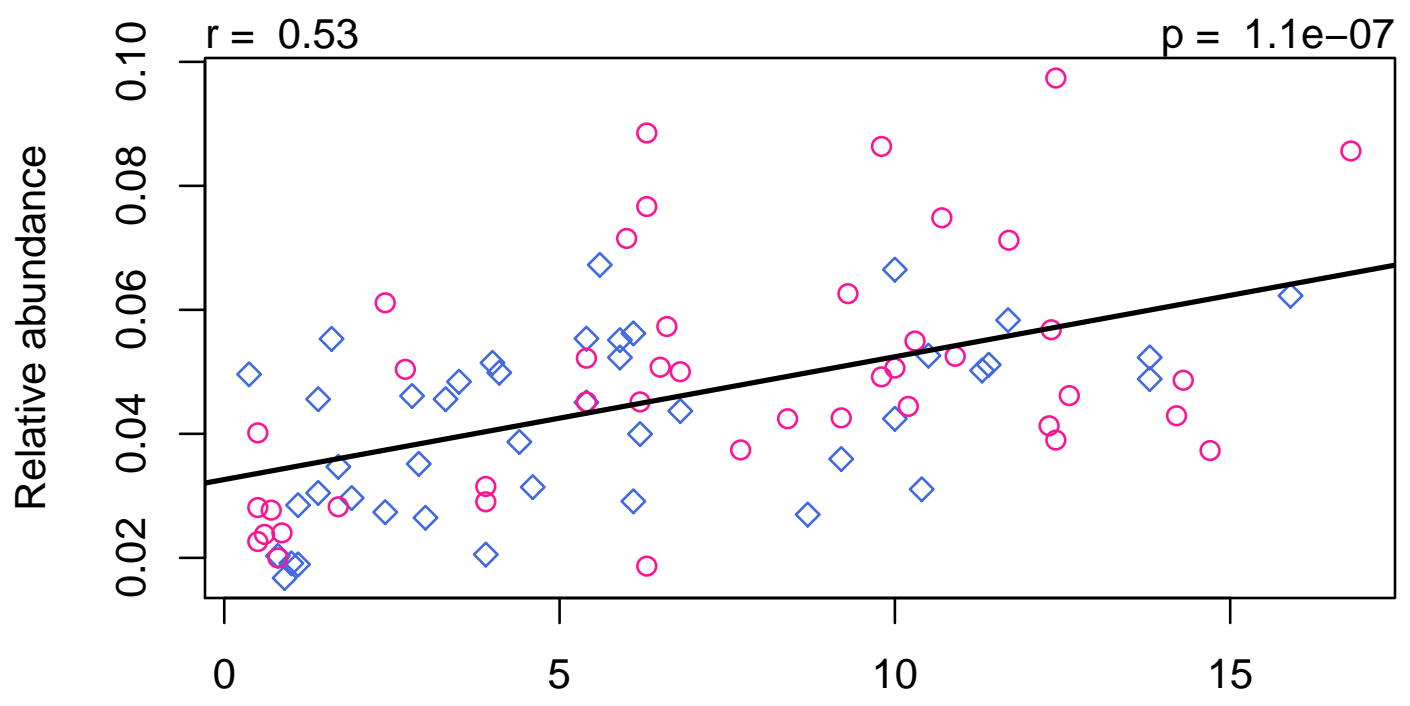

Age (years) 
IgG1_H4N4F1D1

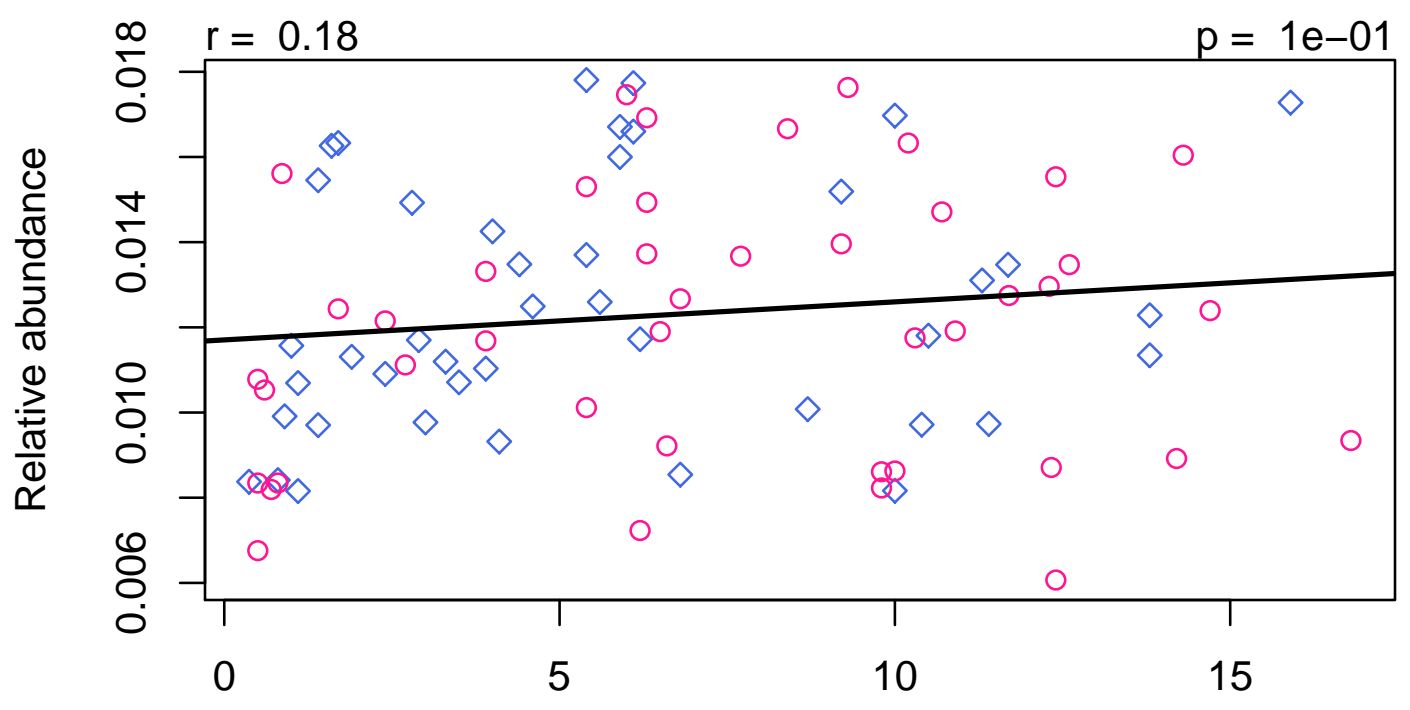

Age (years)

IgG1_H5N4D1

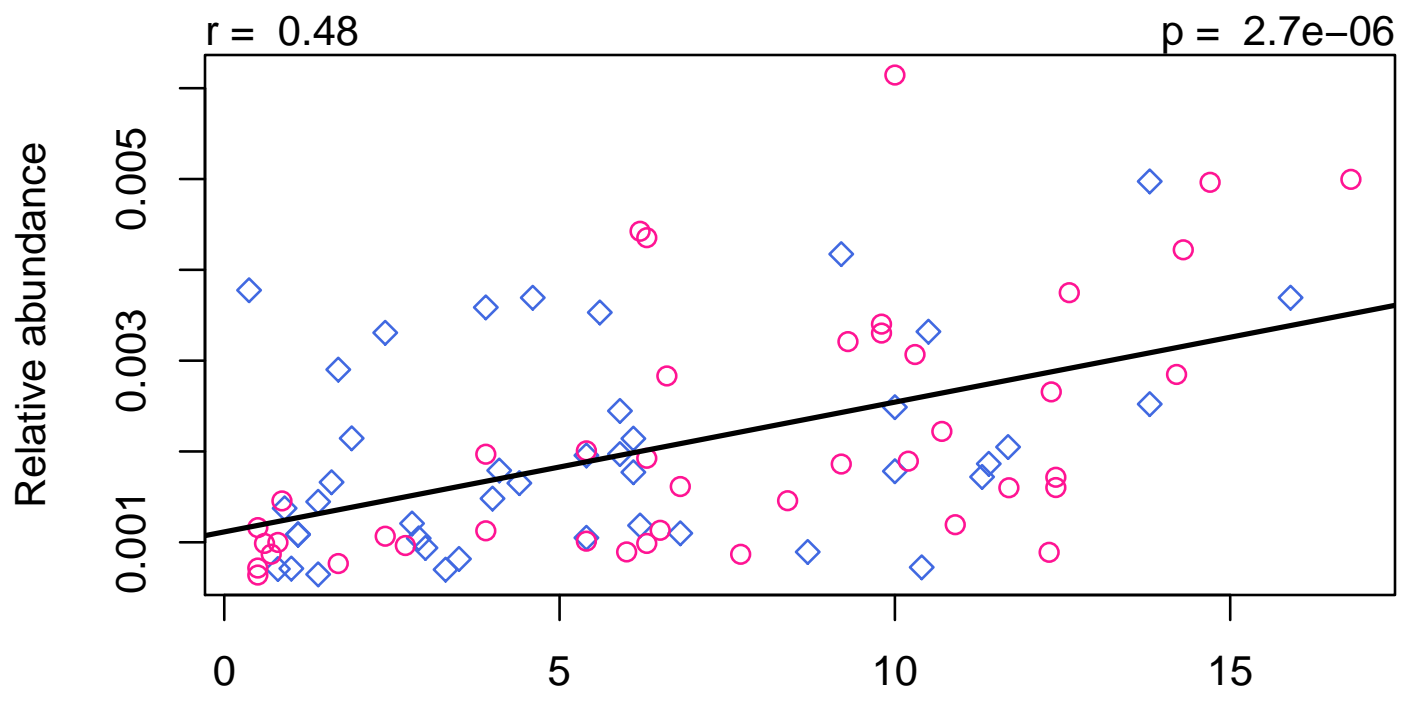

Age (years) 
IgG1_A2

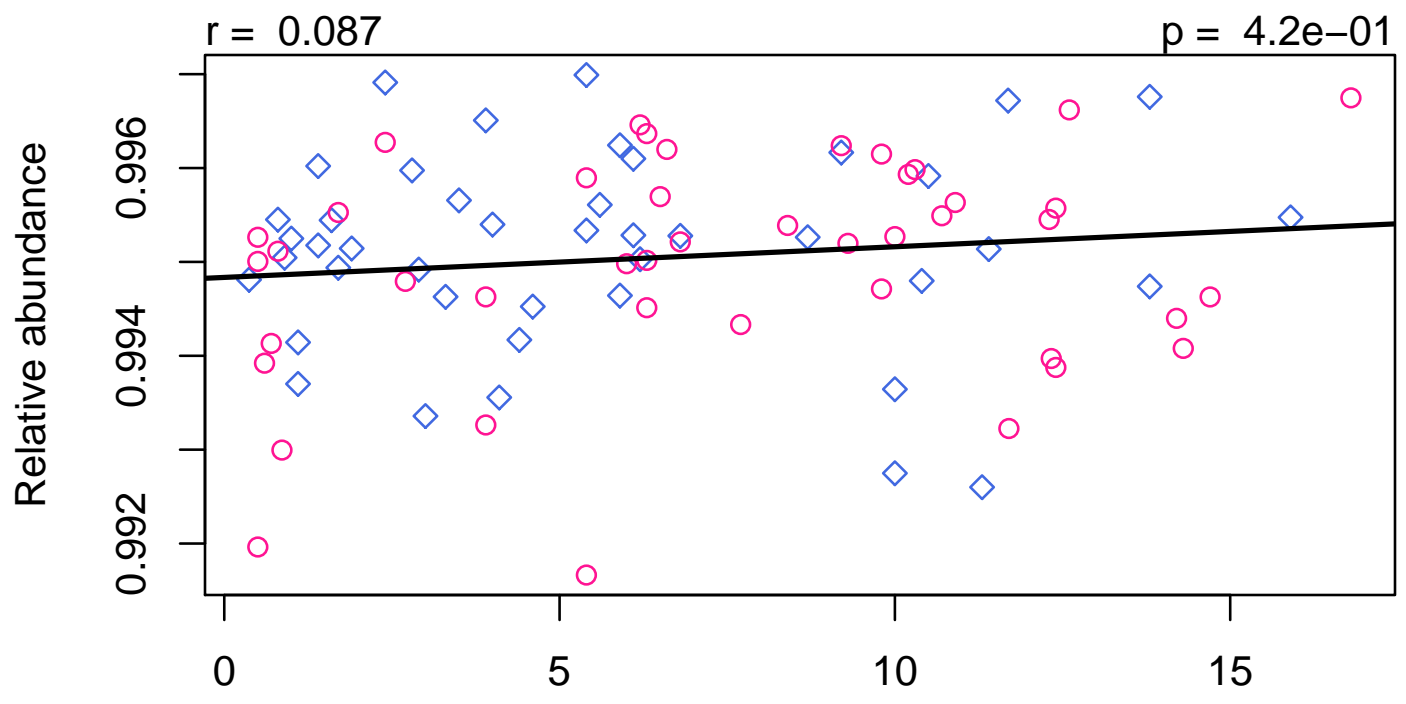

Age (years)

IgG1_B

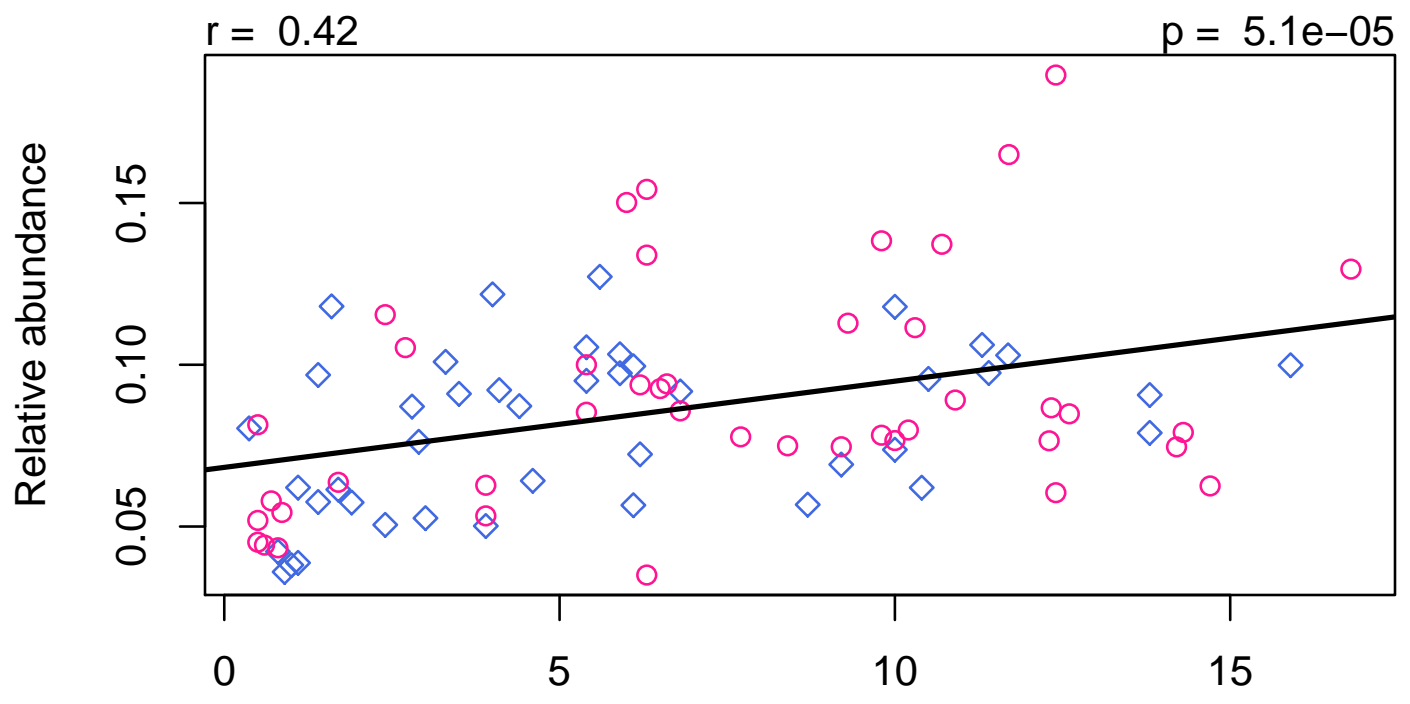


IgG1_F

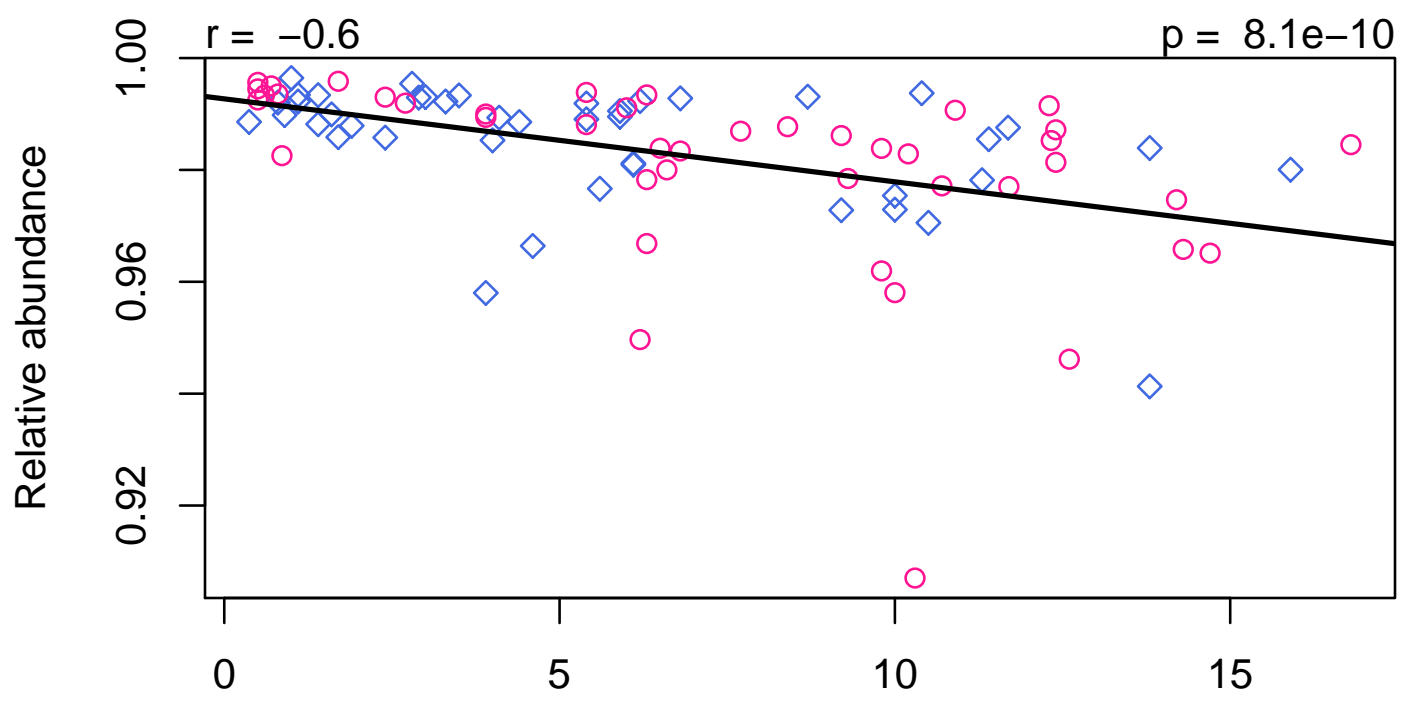

Age (years)

IgG1_A2F

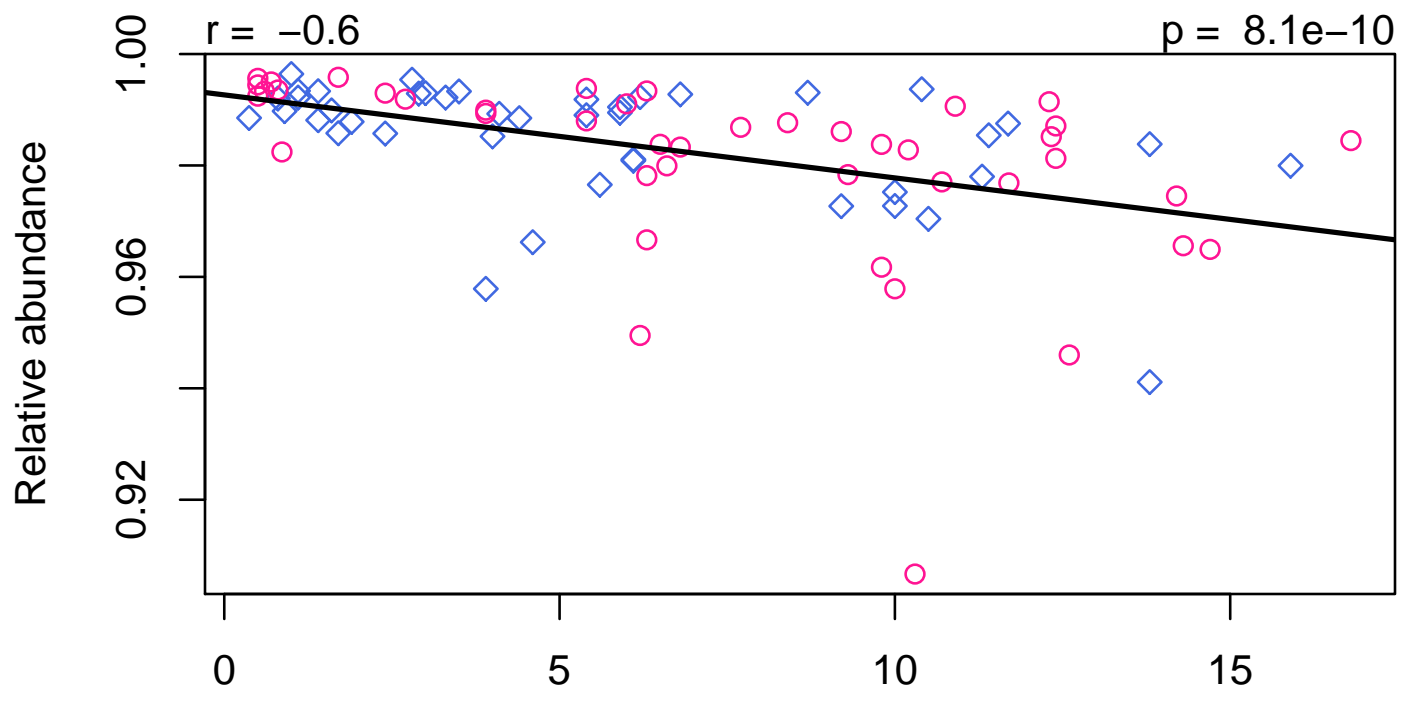


IgG1_A2B

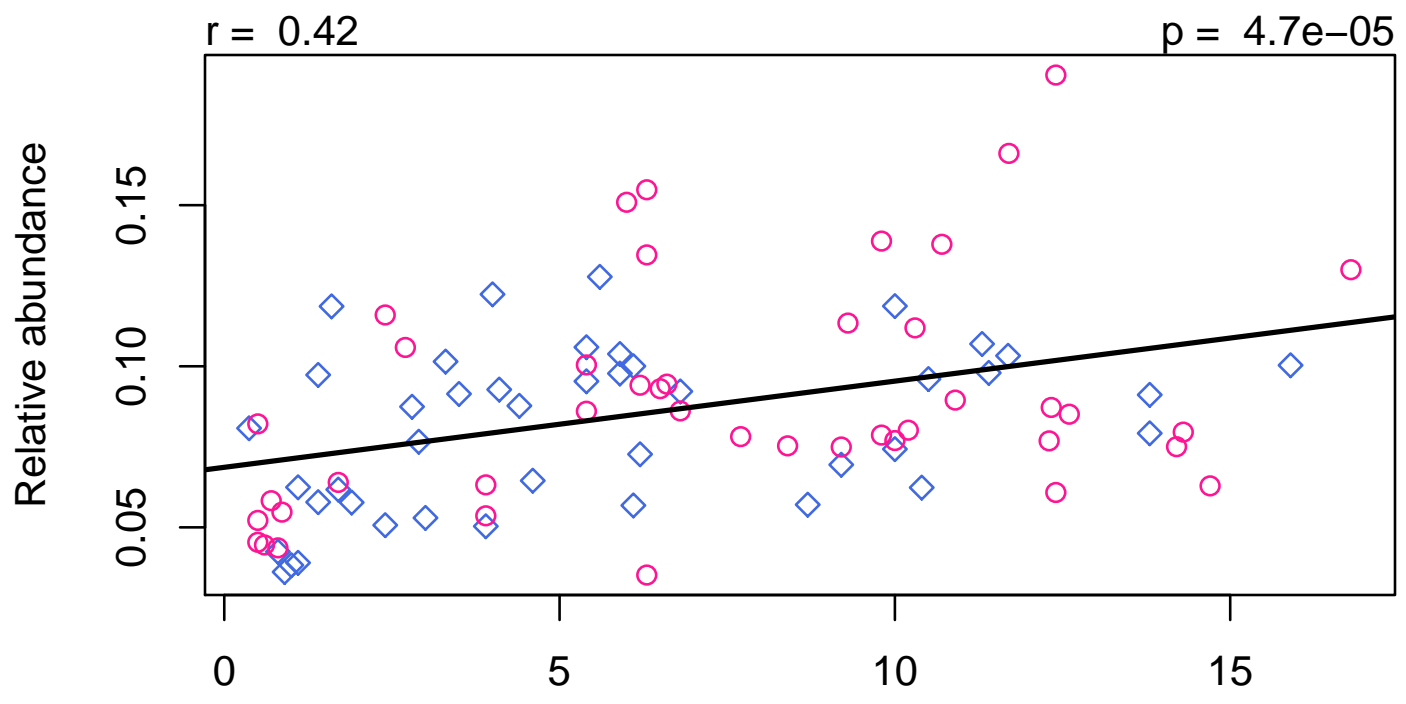

Age (years)

IgG1_A2F0B

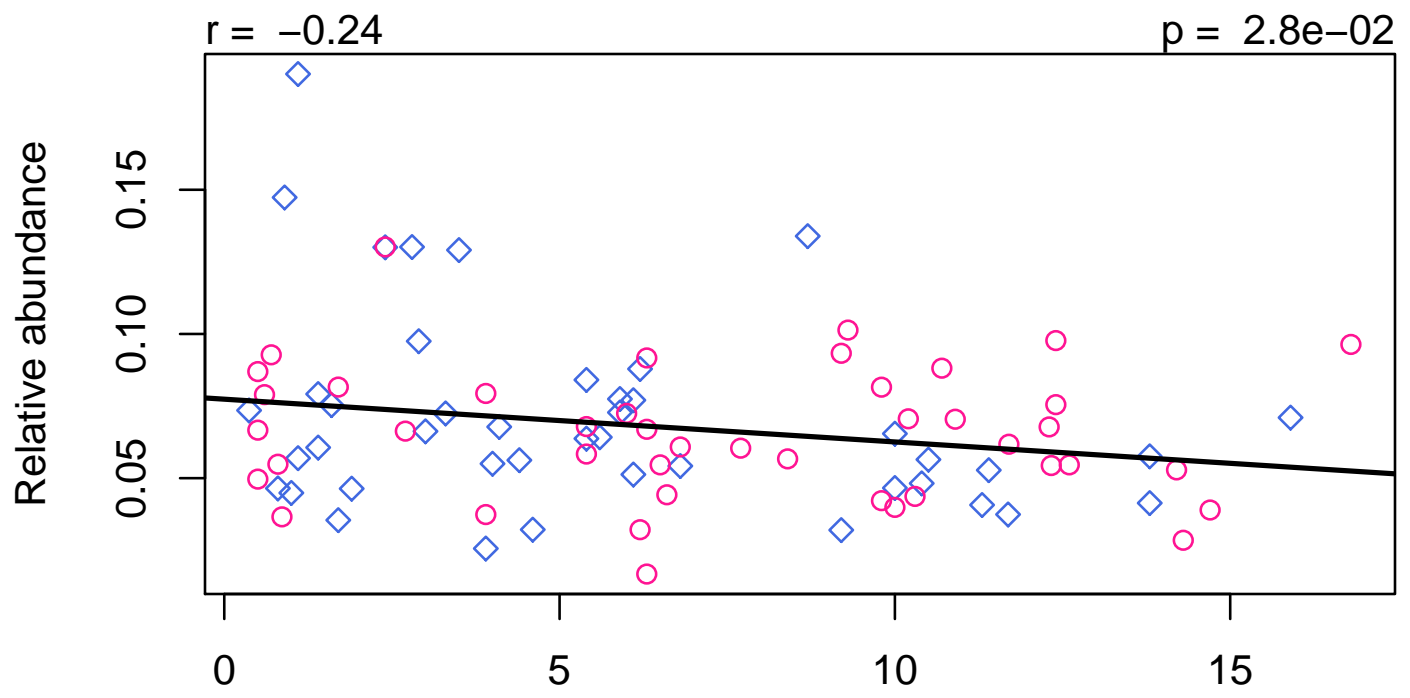

Age (years) 
IgG1_A2F0GD

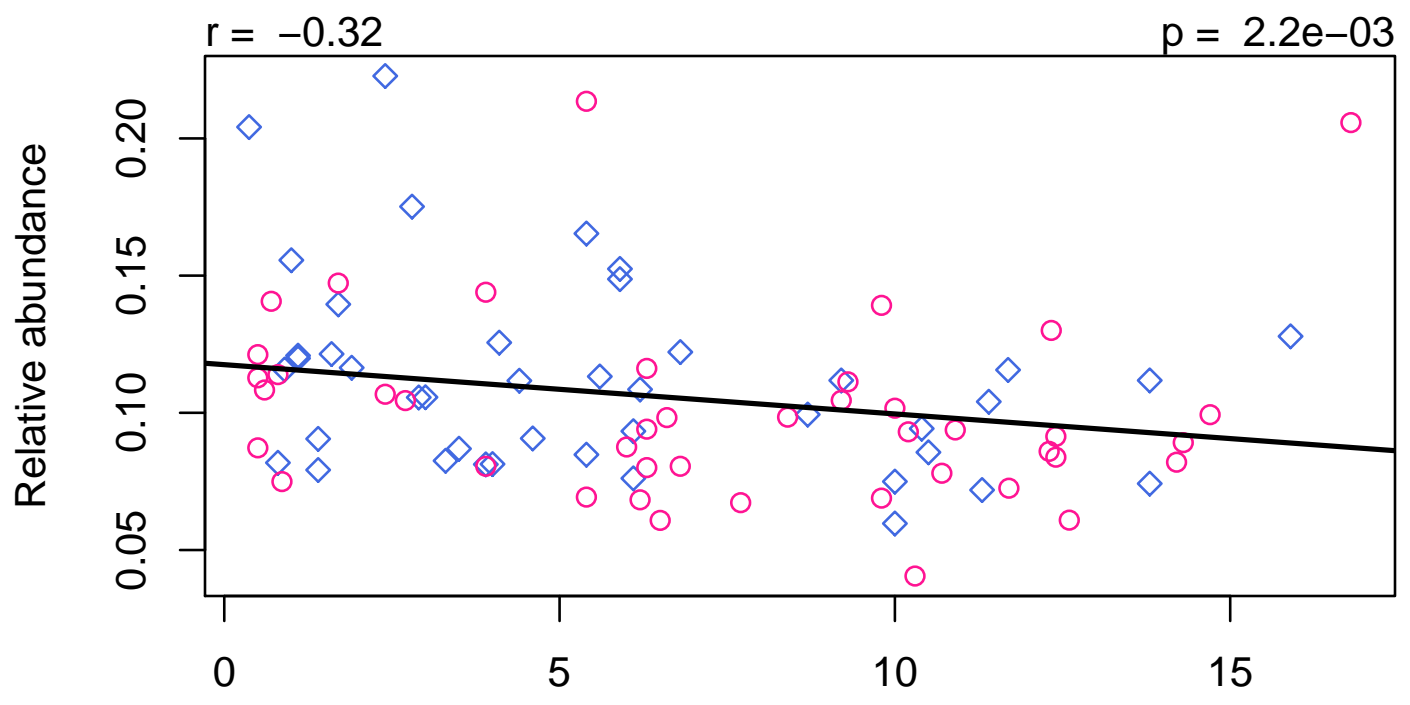

Age (years)

IgG1_A2FGD

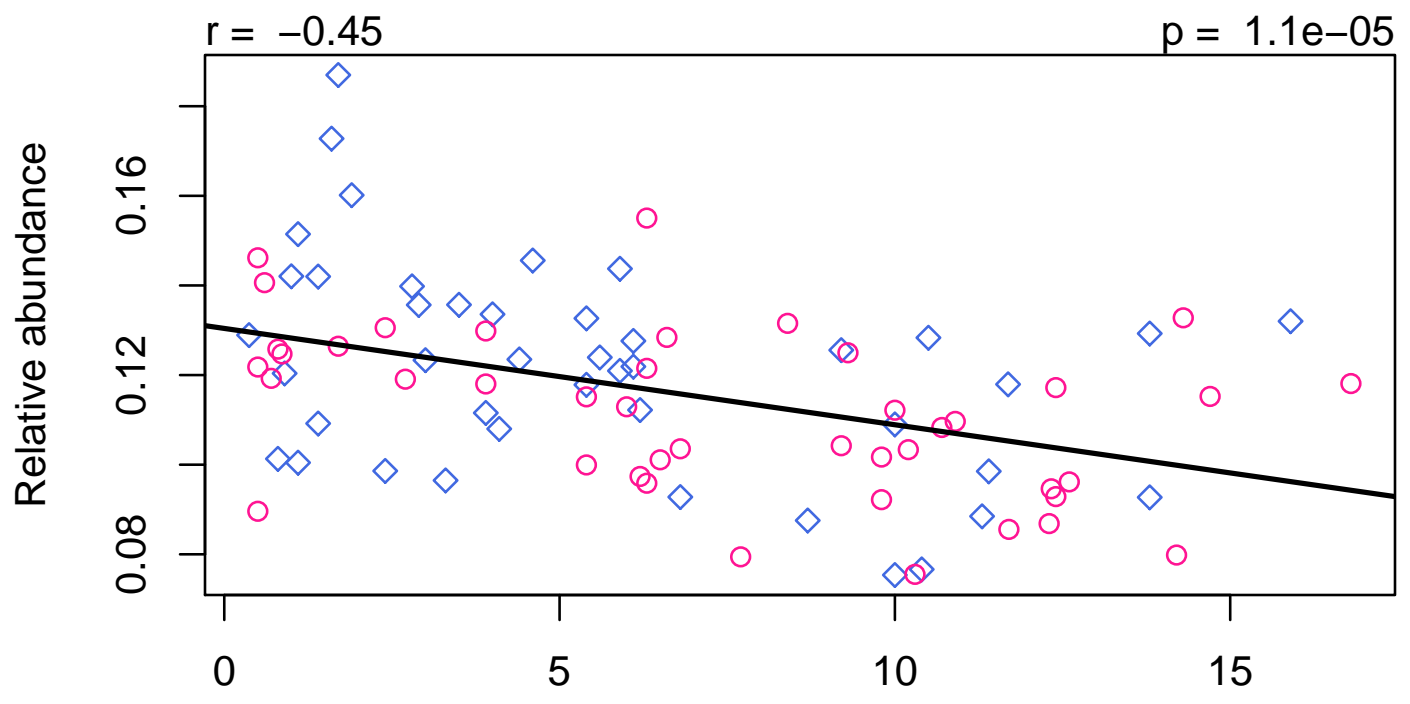

Age (years) 
IgG23_A2FGD

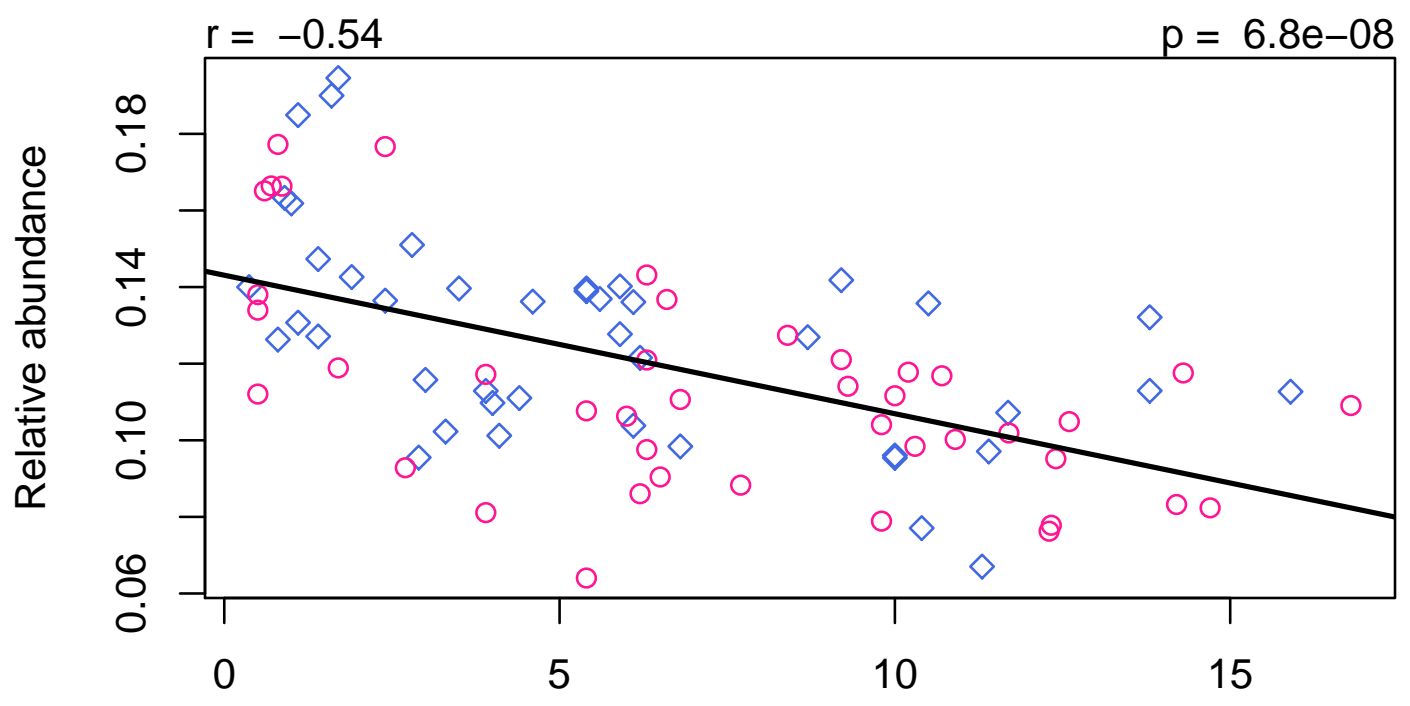

Age (years) 
IgG1_H4N4F1

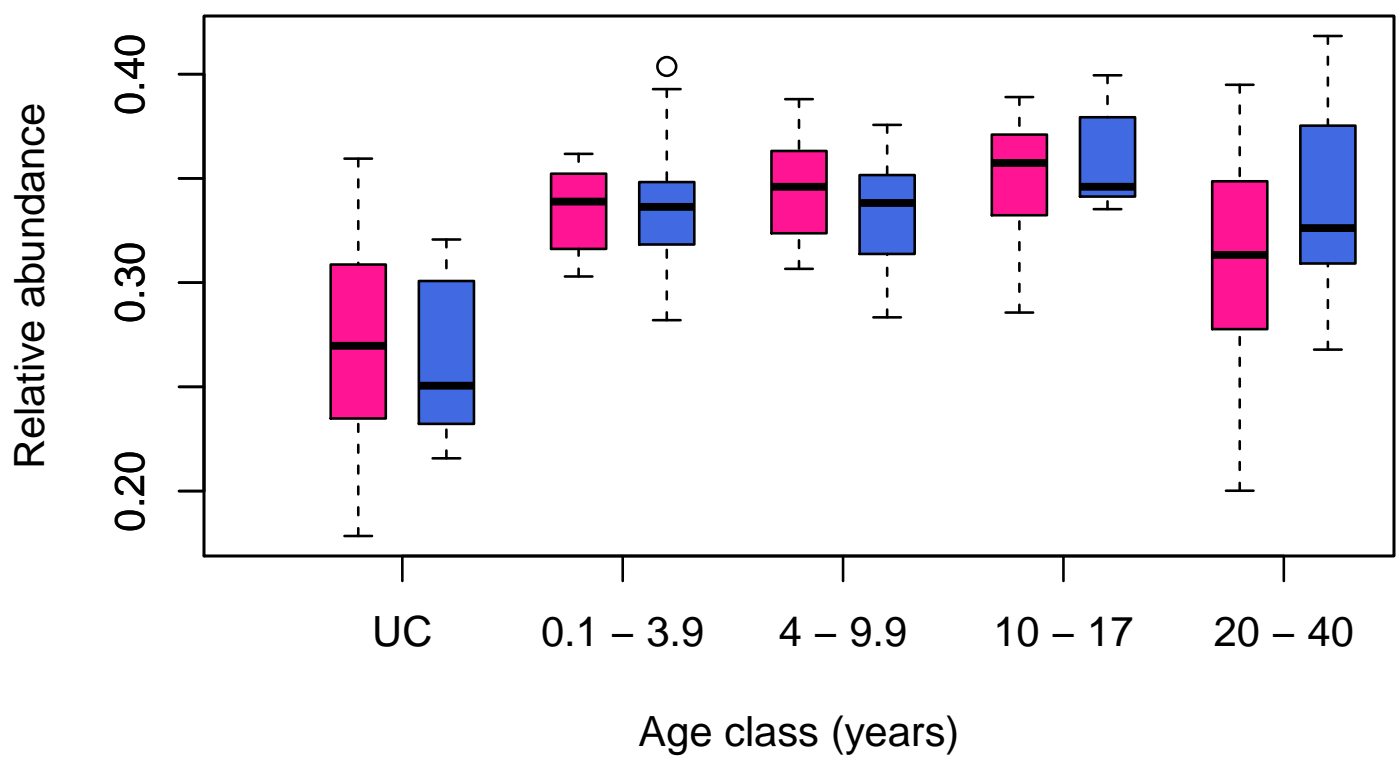

IgG1_H5N4

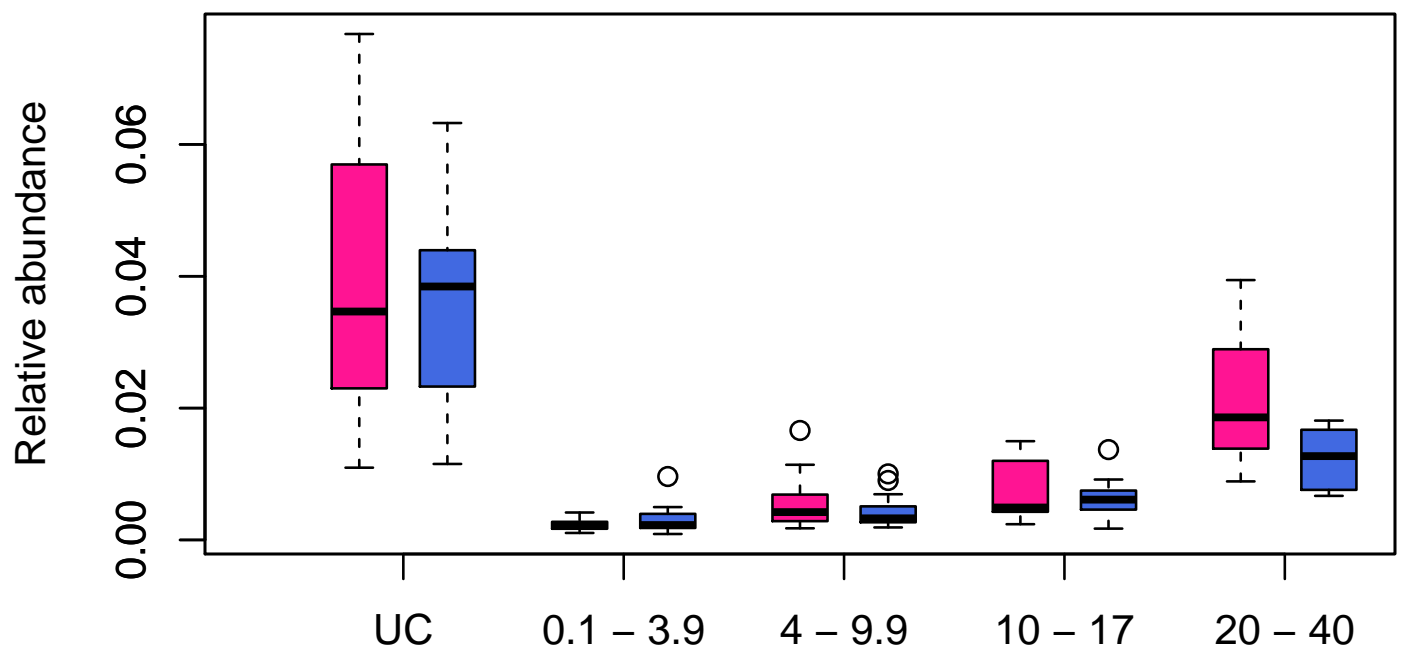

Age class (years) 
IgG1_H3N5F1

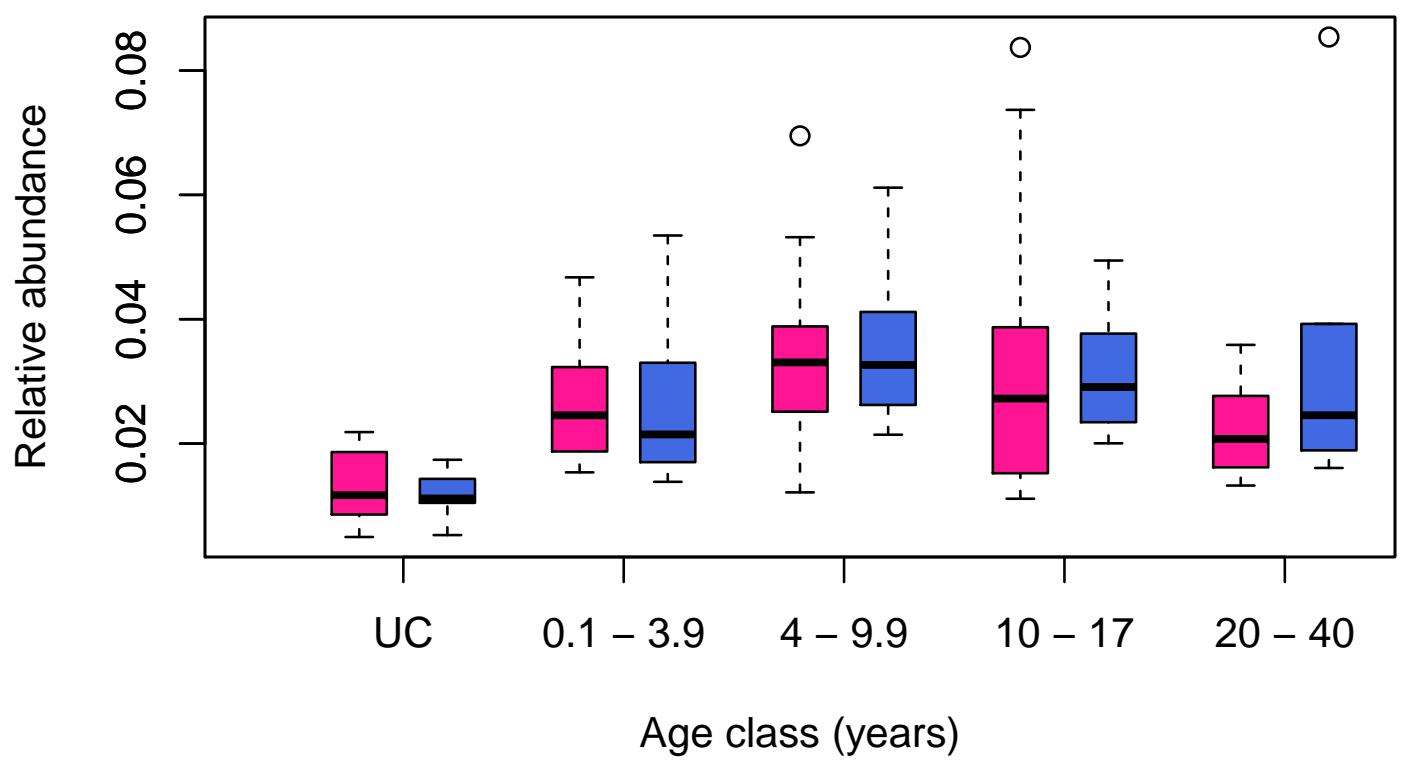

IgG1_H4N5

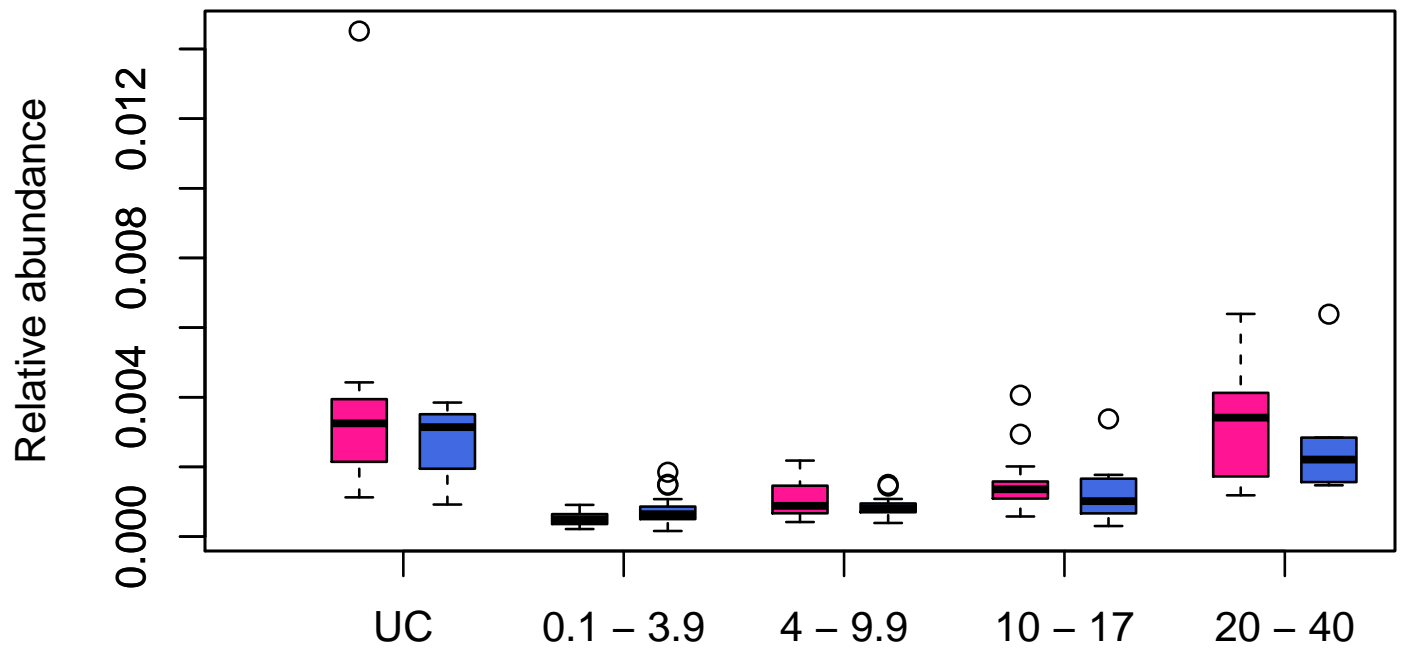

Age class (years) 
IgG1_H4N4F1D1

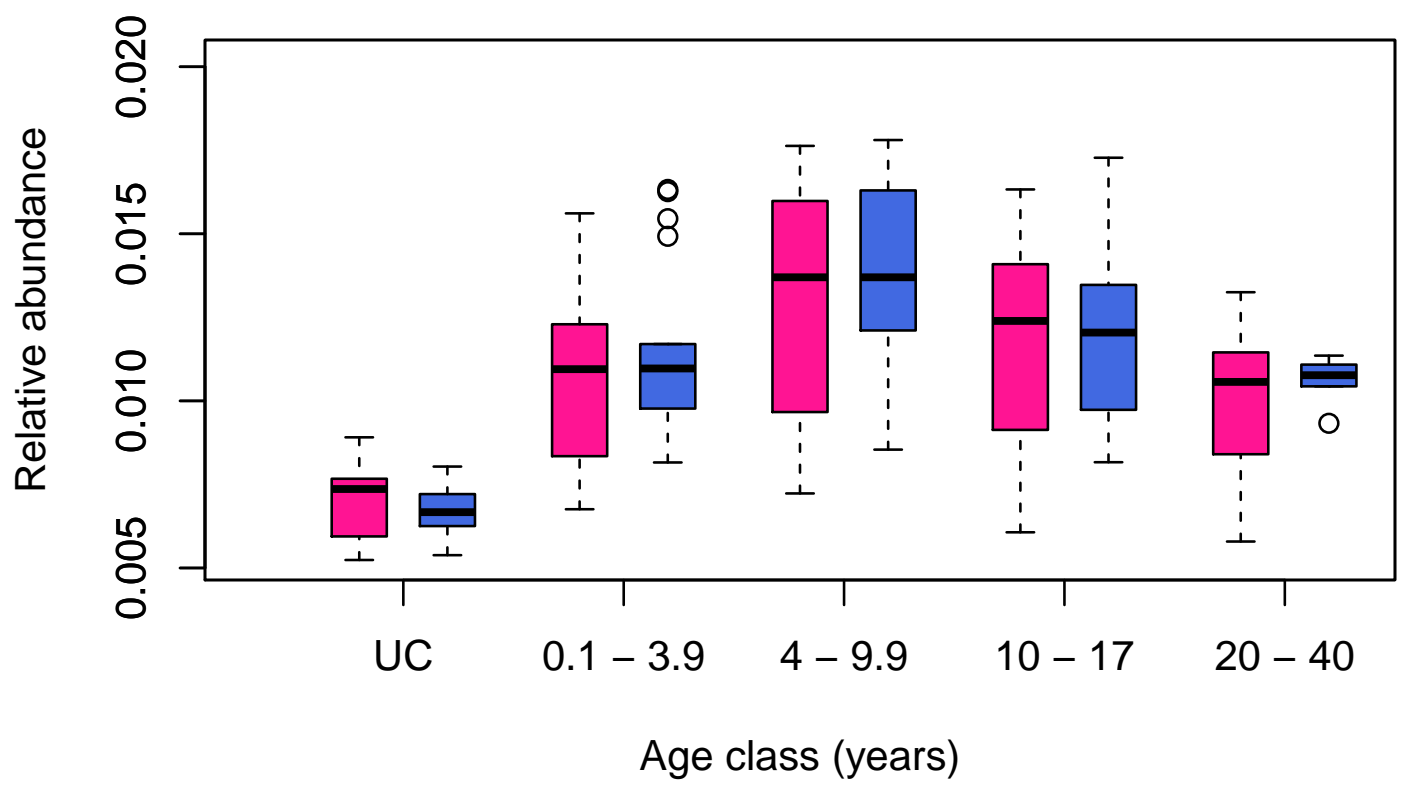

IgG1_H5N4D1

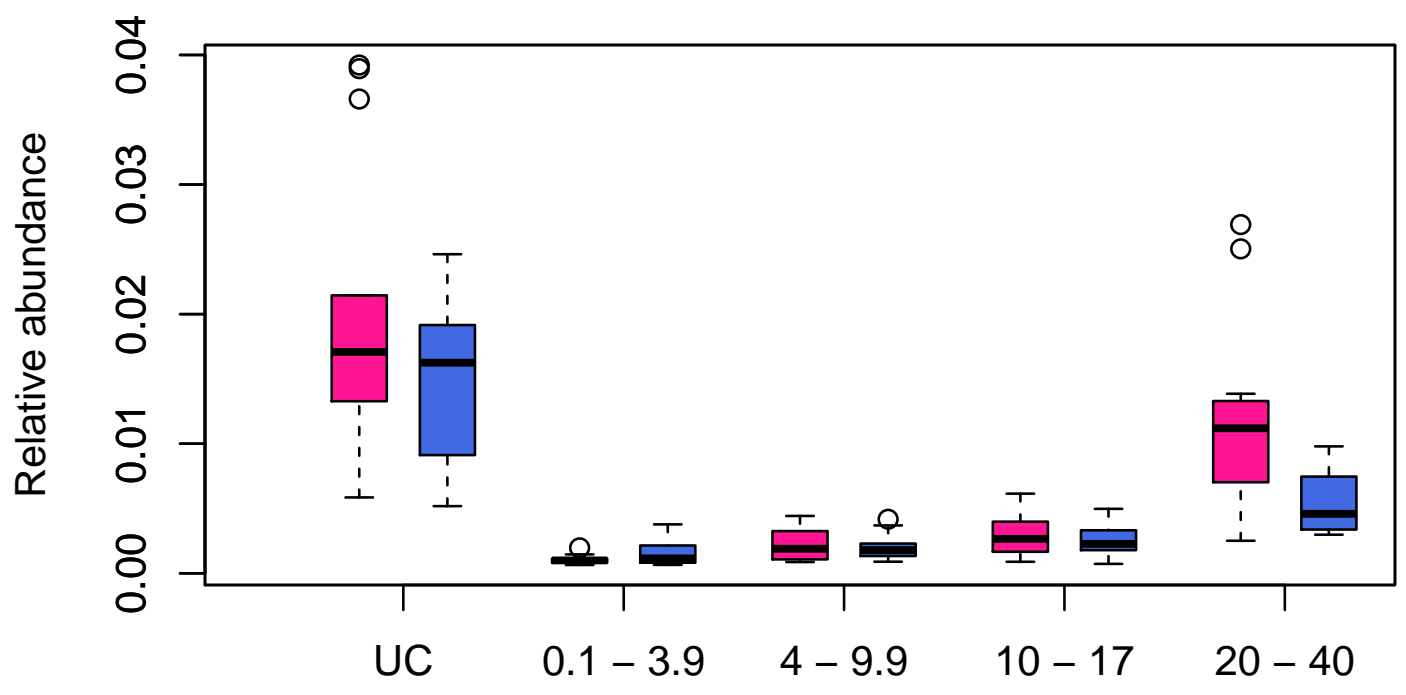

Age class (years) 
IgG1_H5N5F1

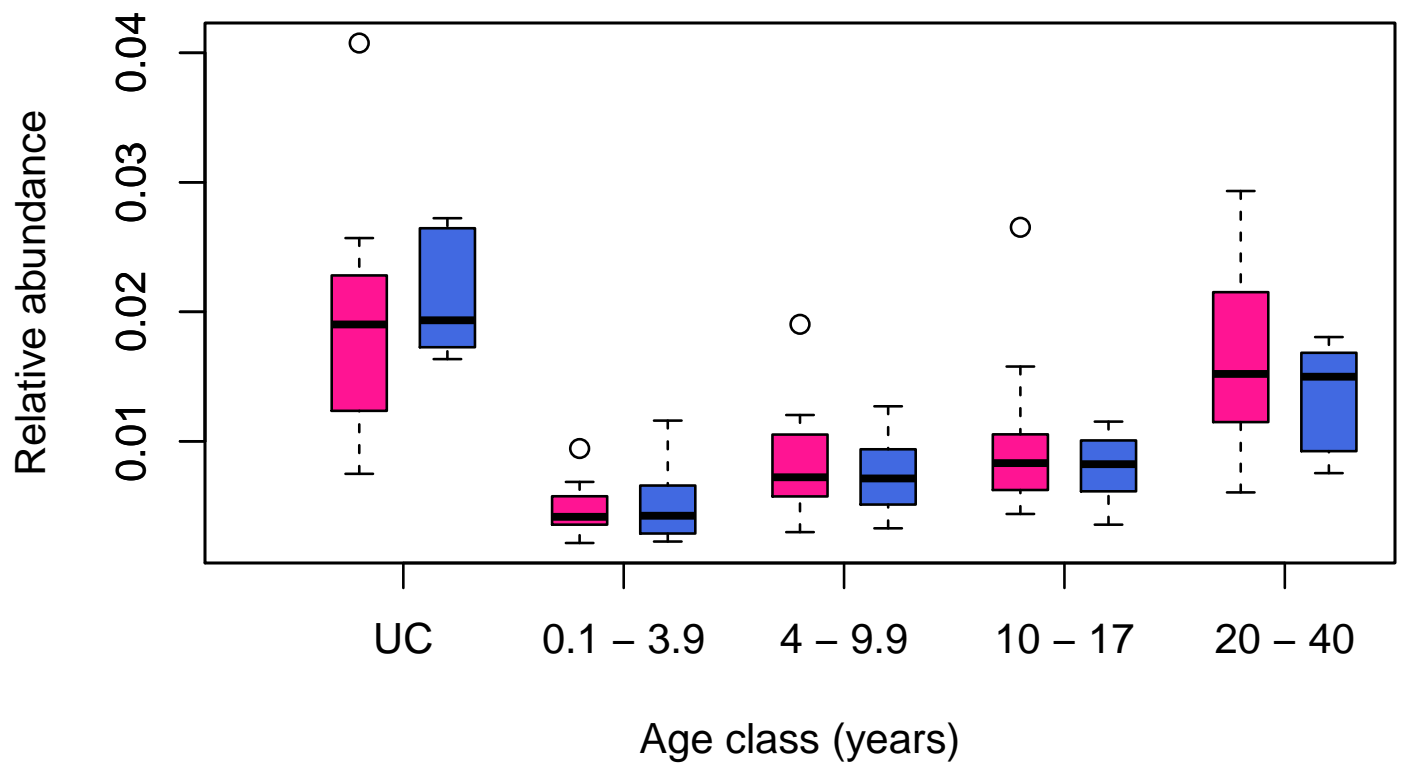

IgG1_H5N4F1D1

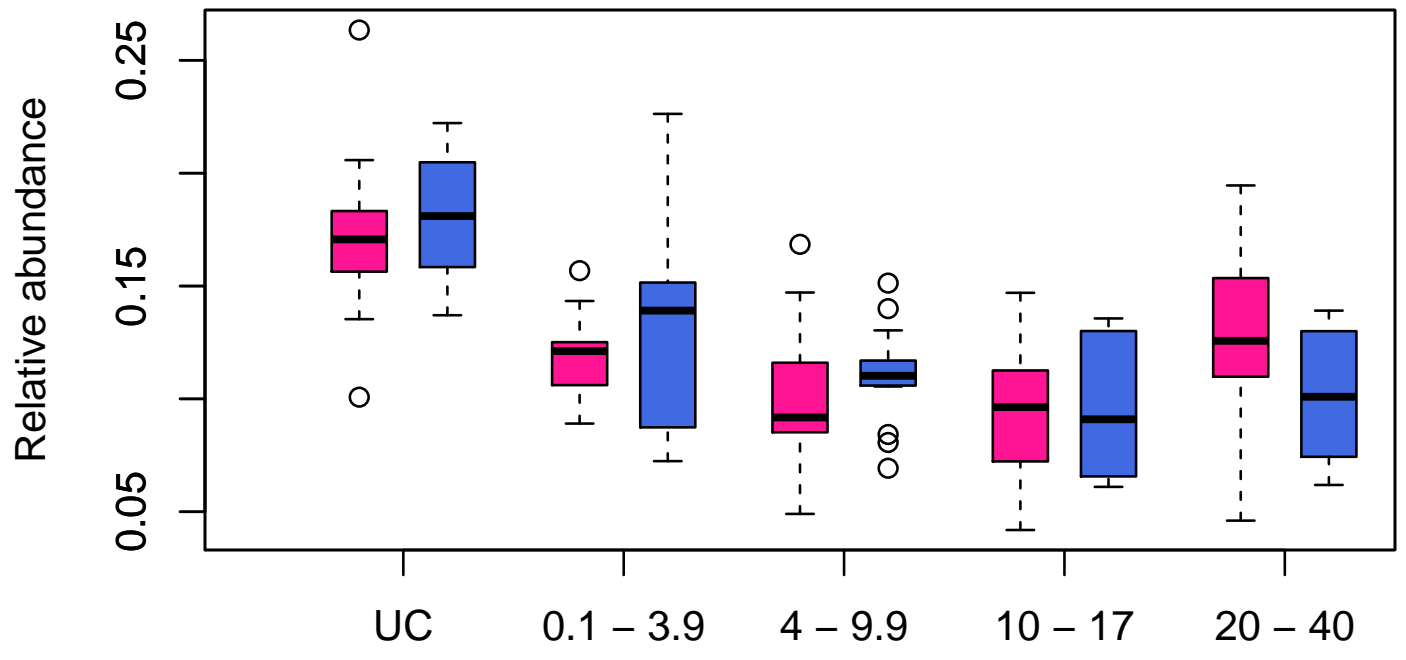

Age class (years) 
IgG1_H5N5F1D1

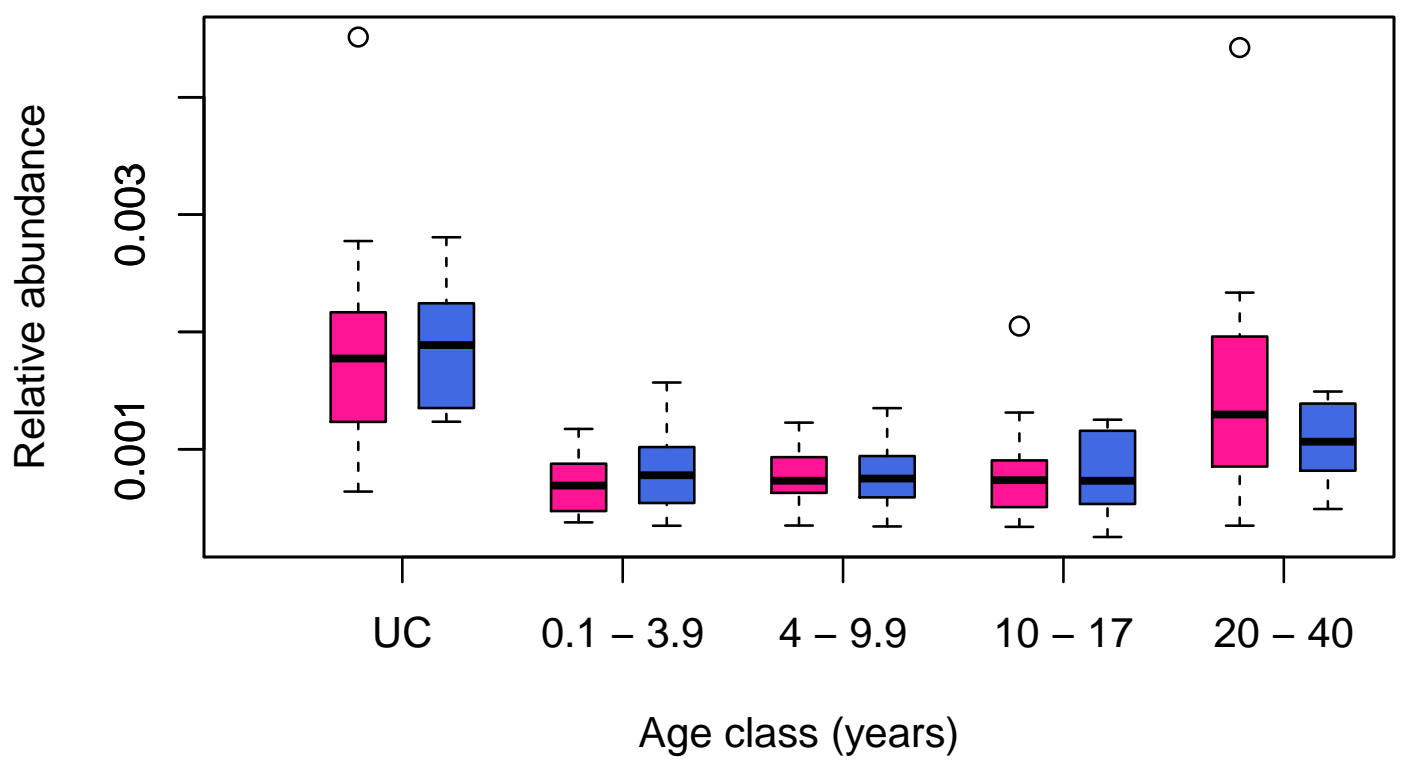

IgG1_H5N4F1D2

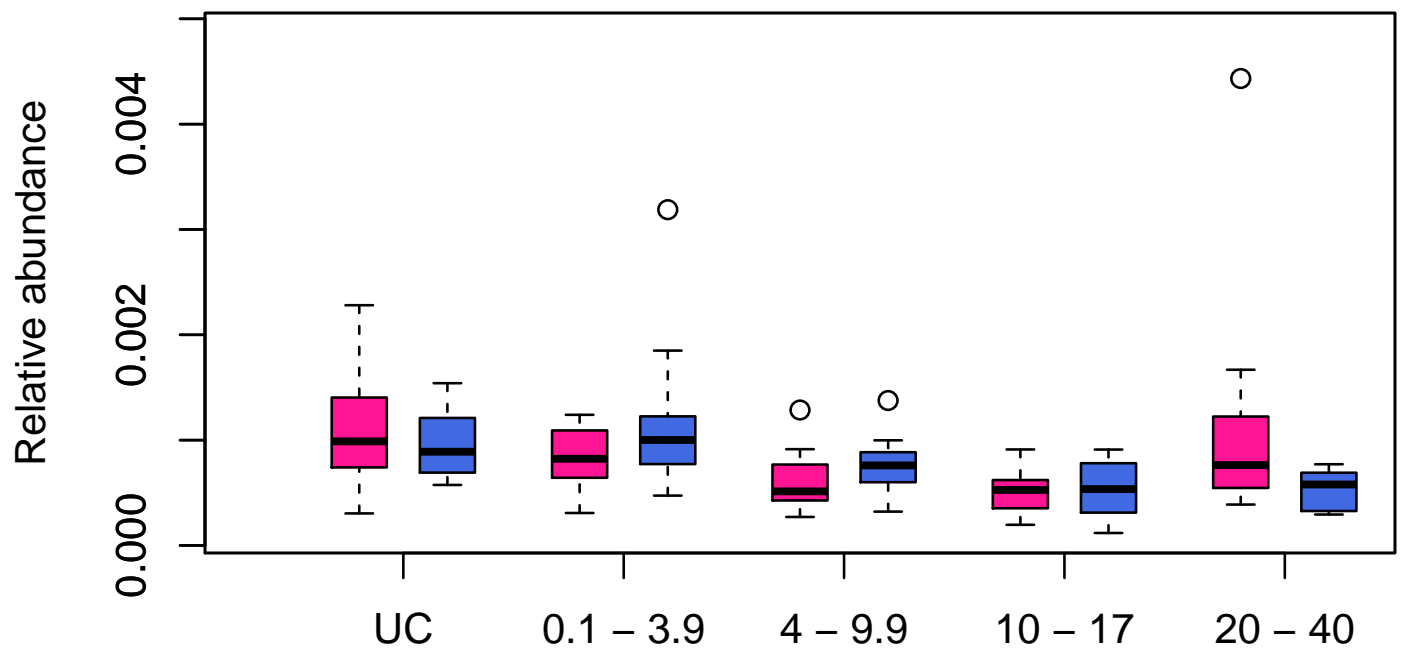

Age class (years) 
IgG23_H3N4F1

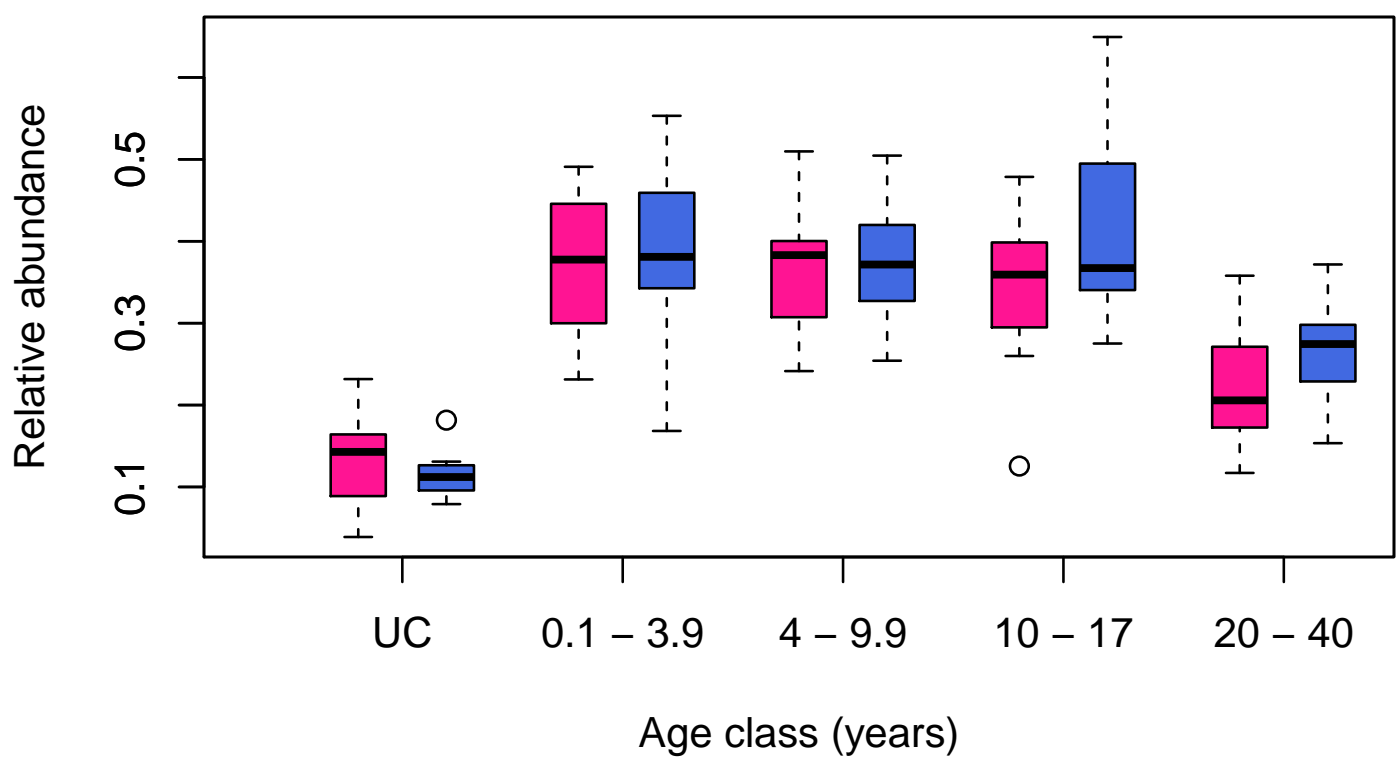

IgG23_H4N4F1

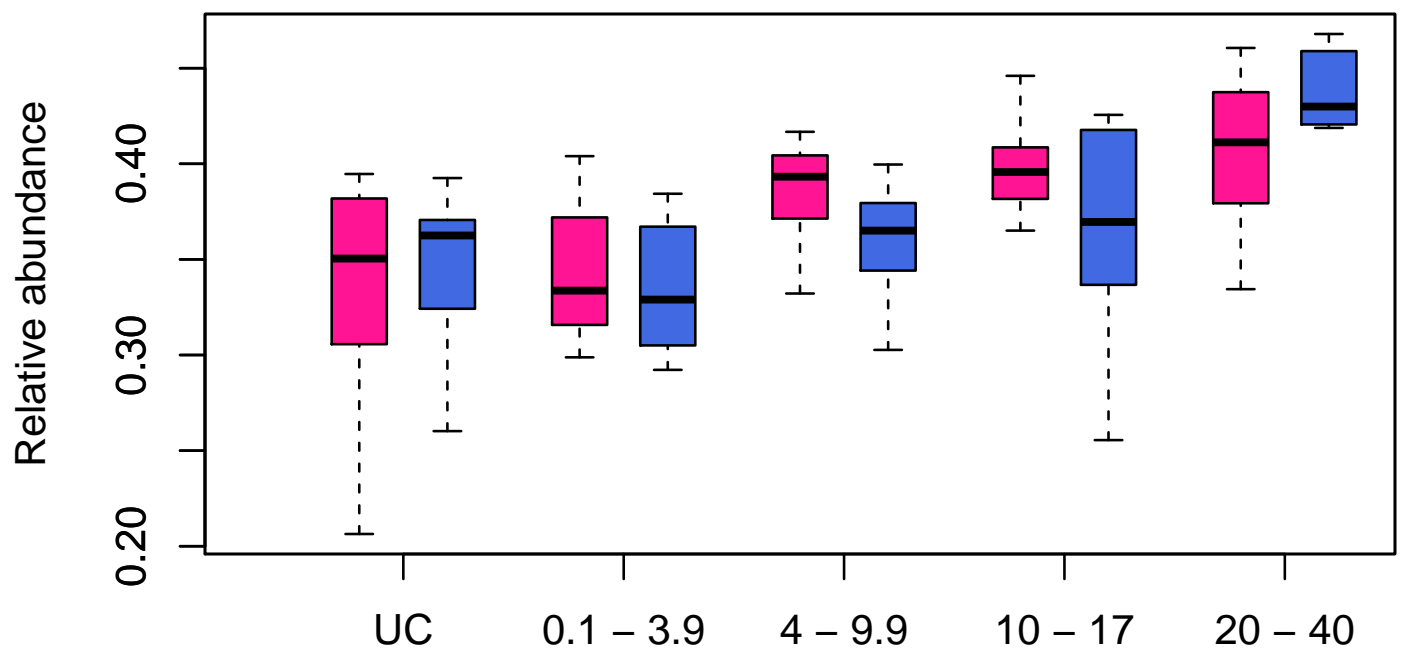

Age class (years) 
IgG23_H5N4F1

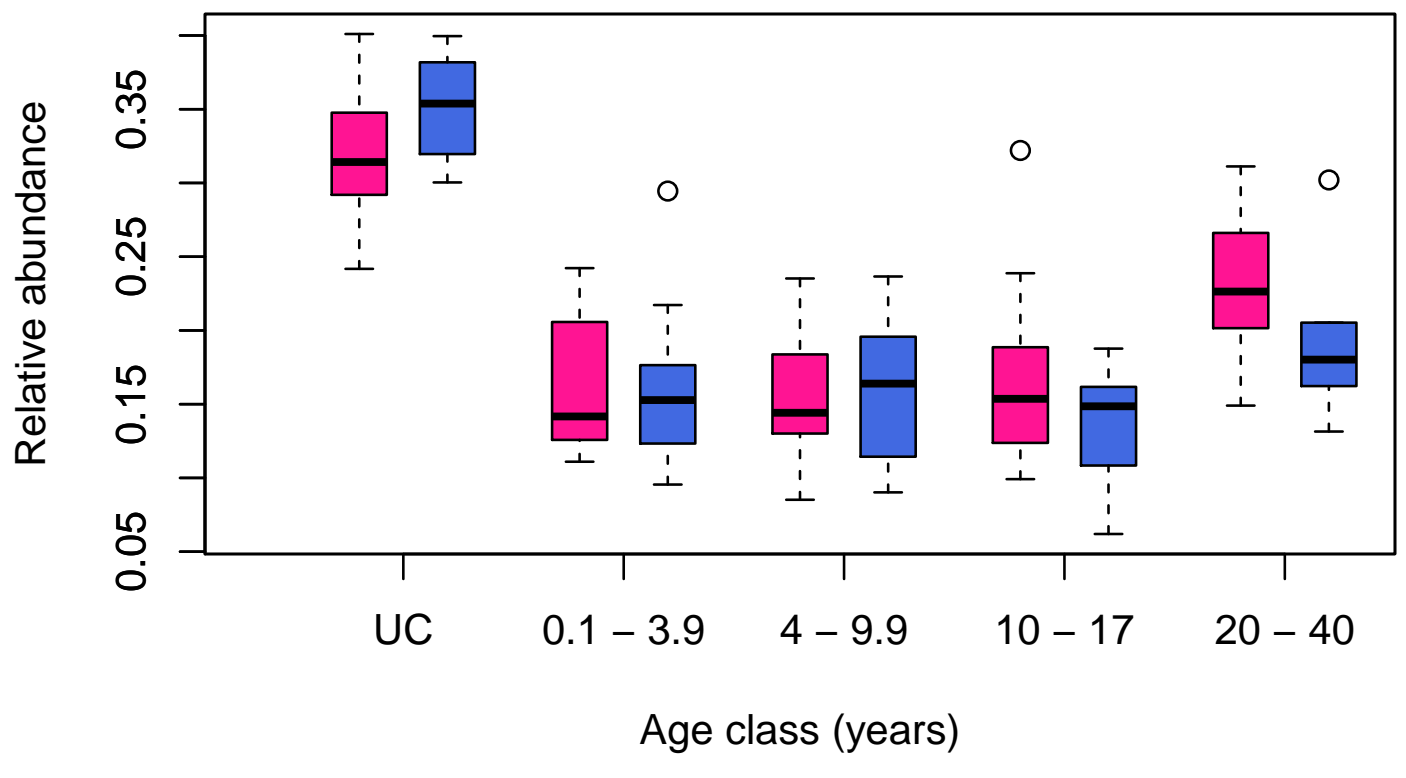

IgG23_H4N4F1D1

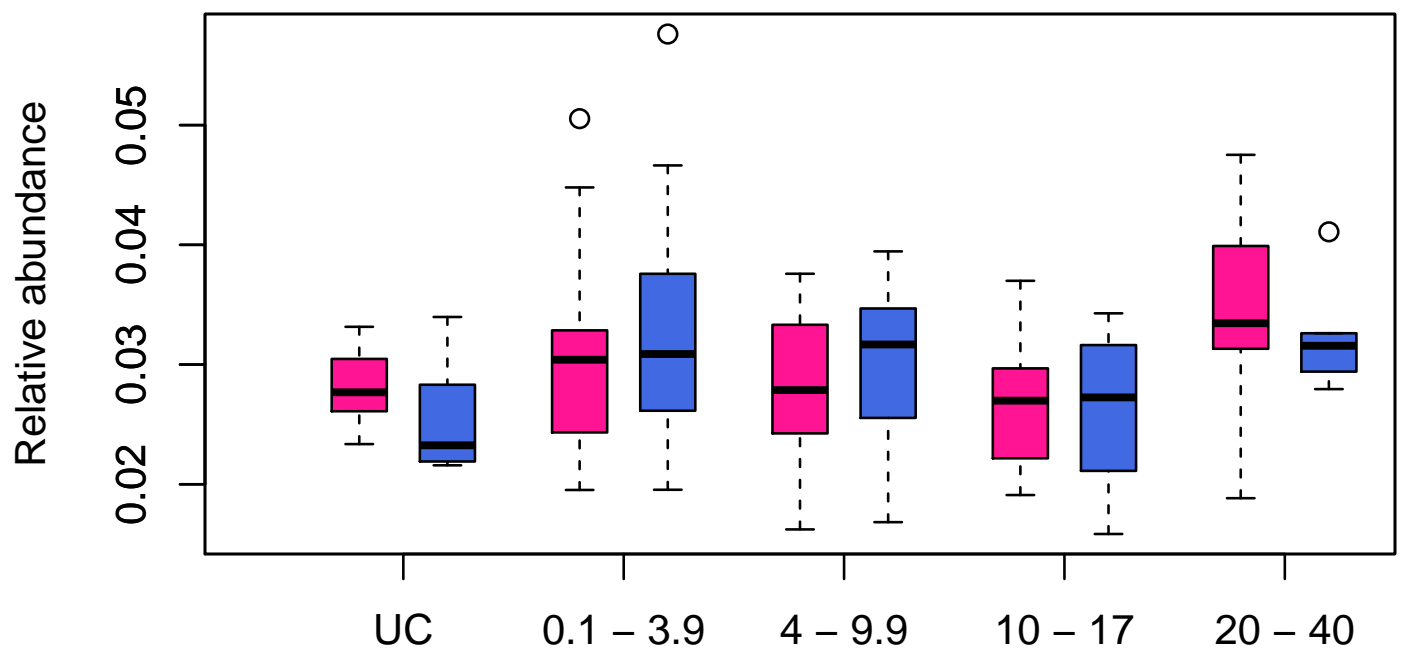

Age class (years) 
IgG1_A2

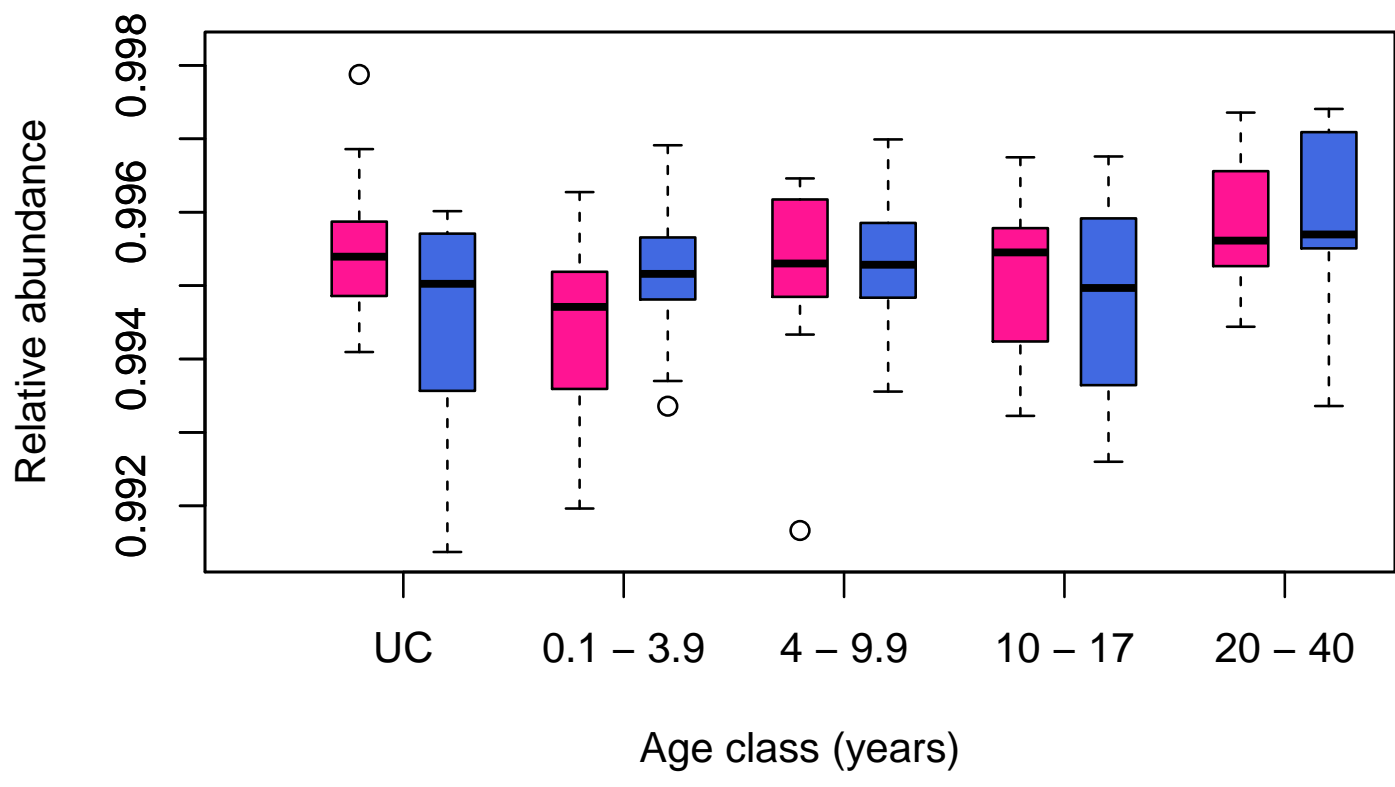

IgG1_B

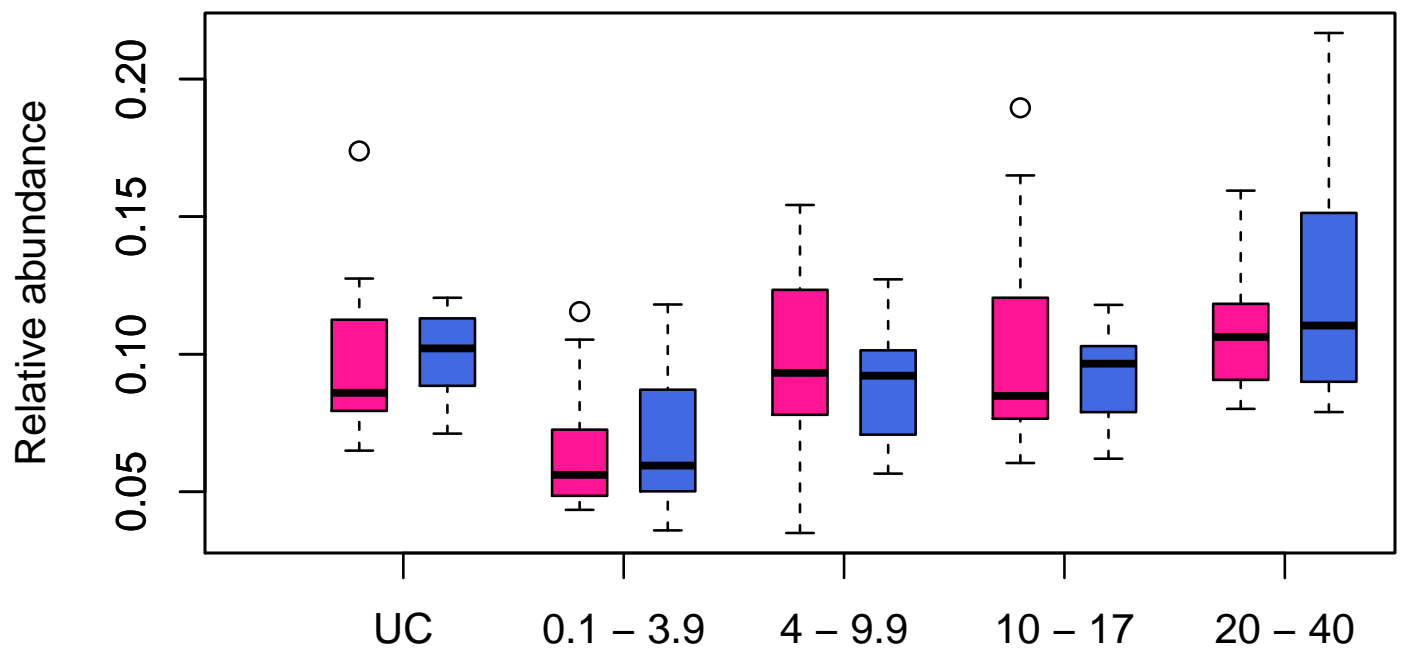

Age class (years) 
IgG1_F

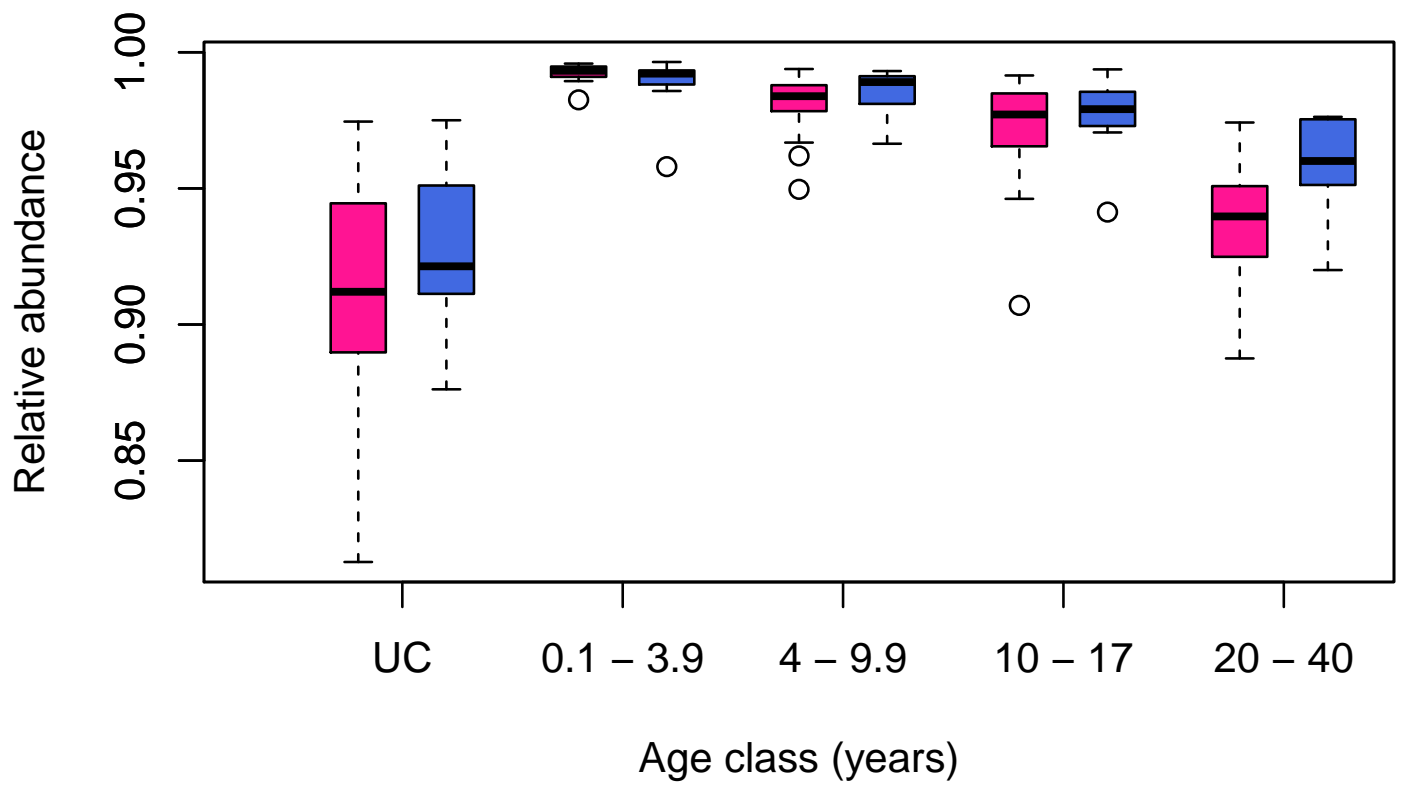

\section{IgG1_A2F}

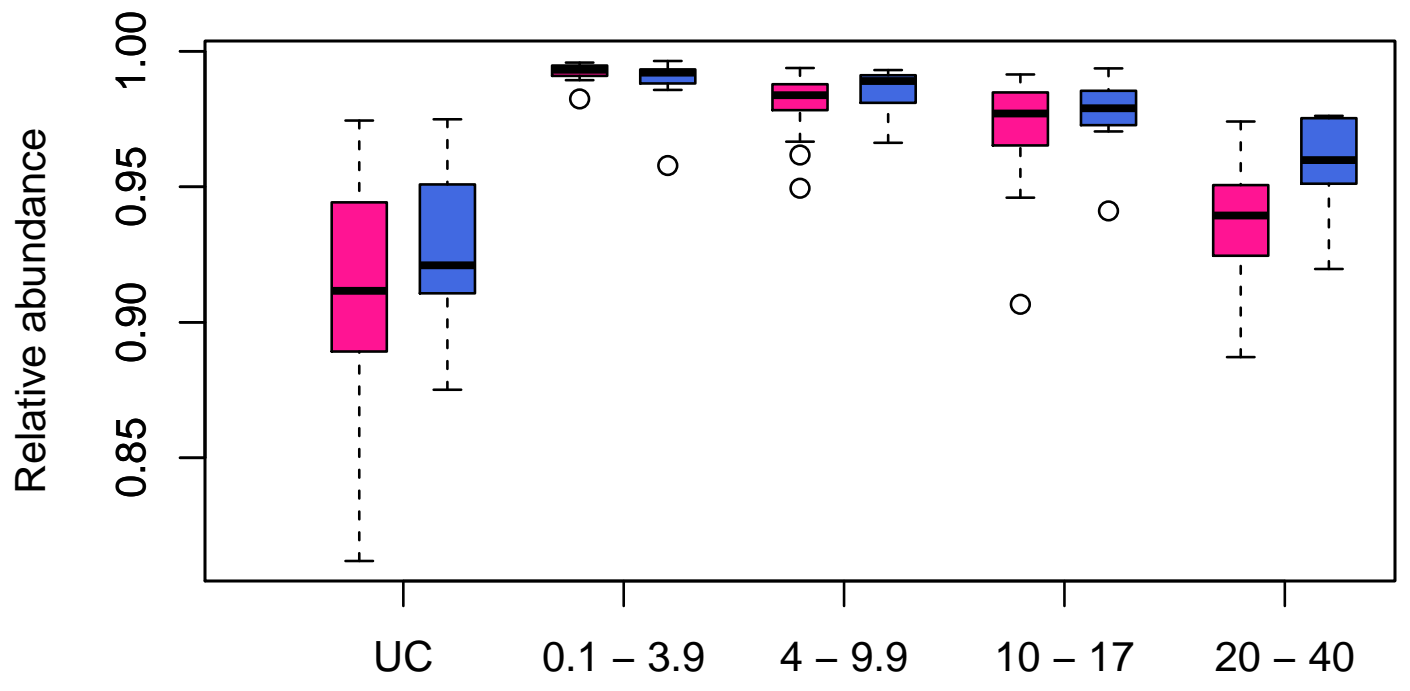

Age class (years) 
IgG1_A2B

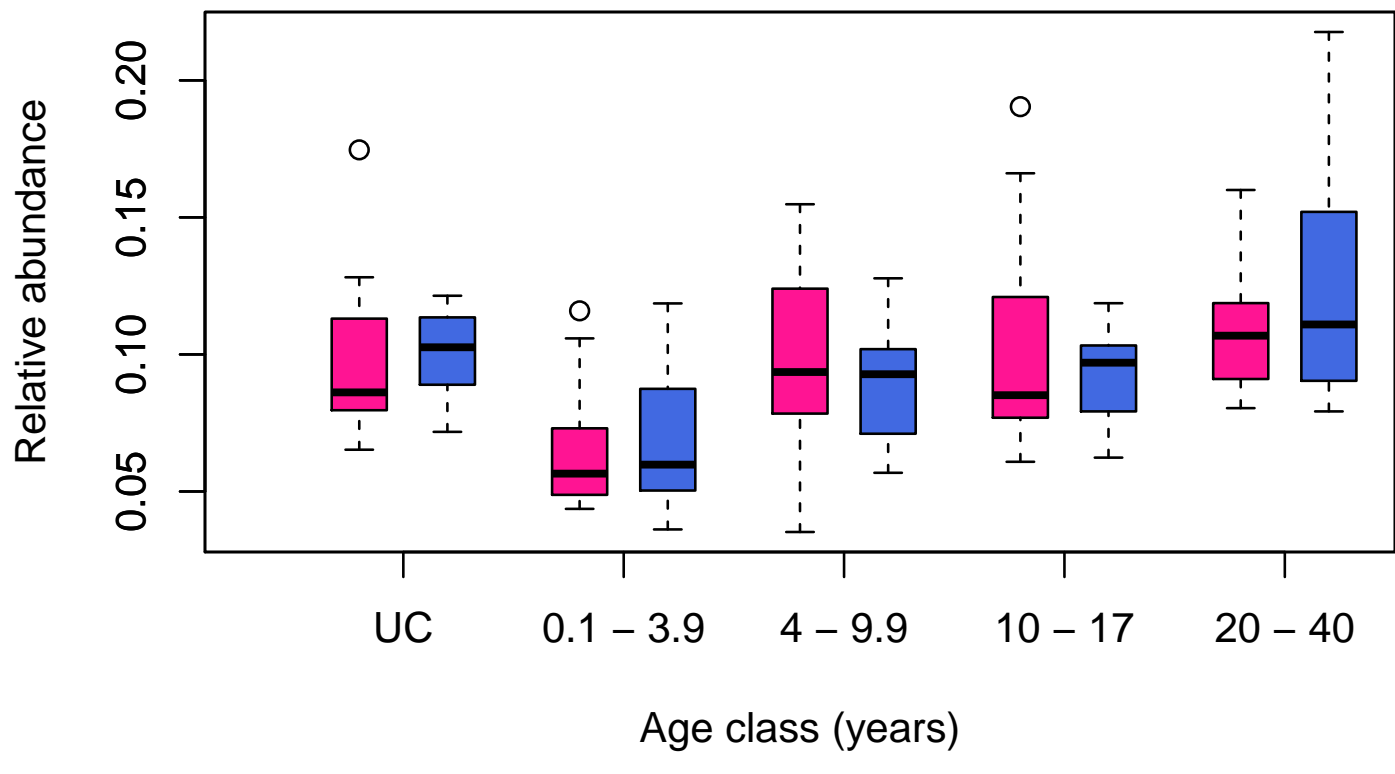

IgG1_A2F0B

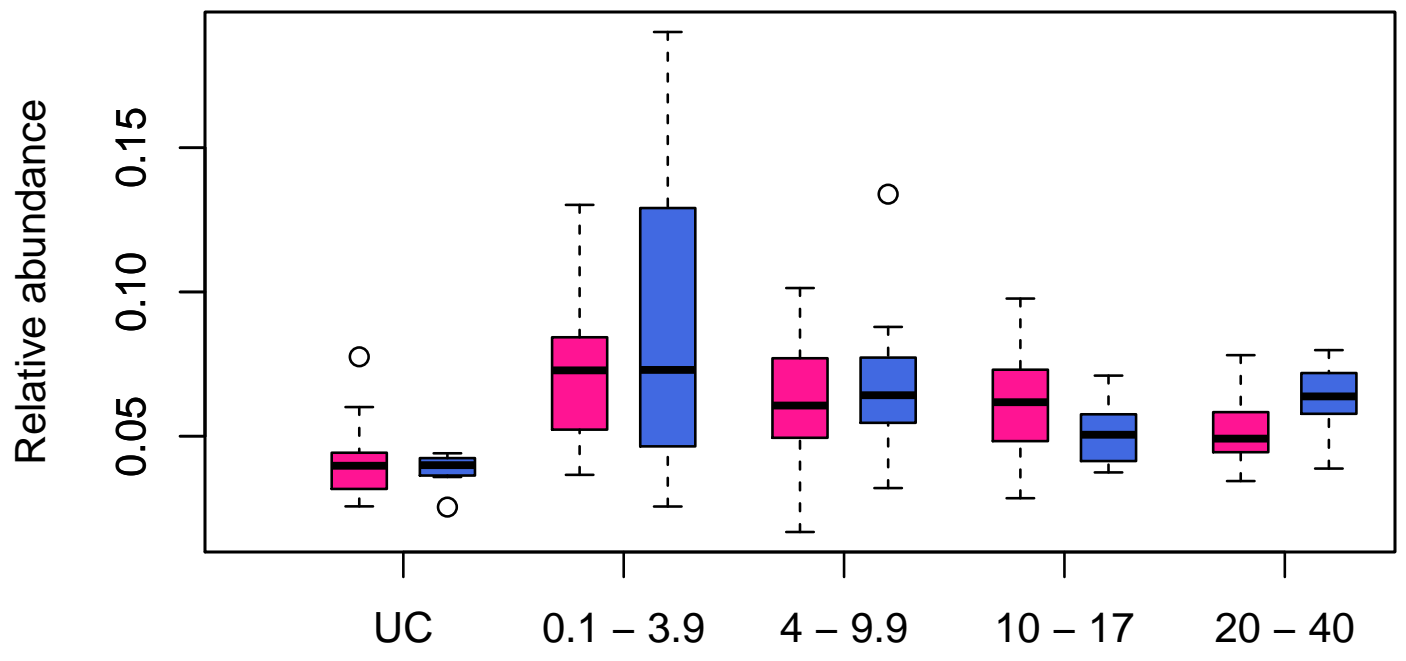

Age class (years) 


\section{IgG1_A2FB}

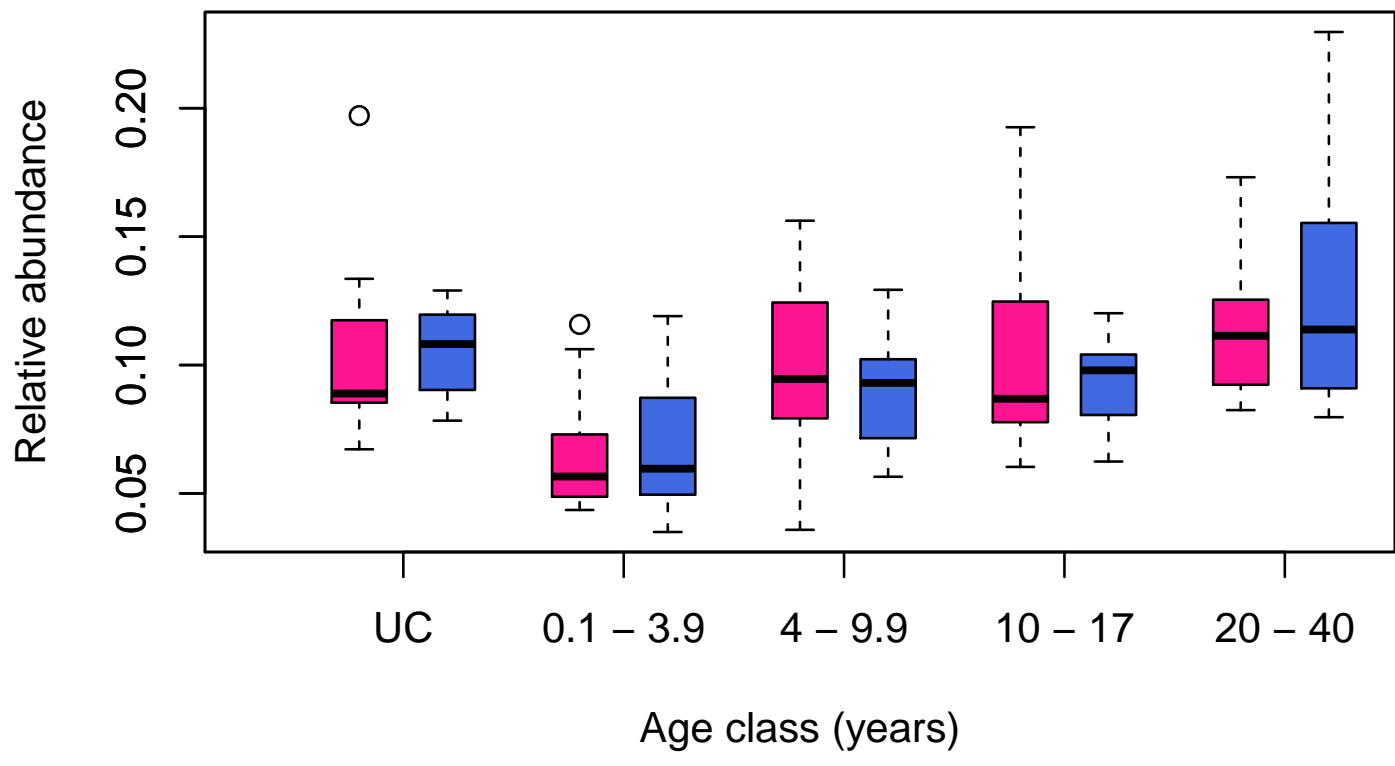

IgG1_A2G

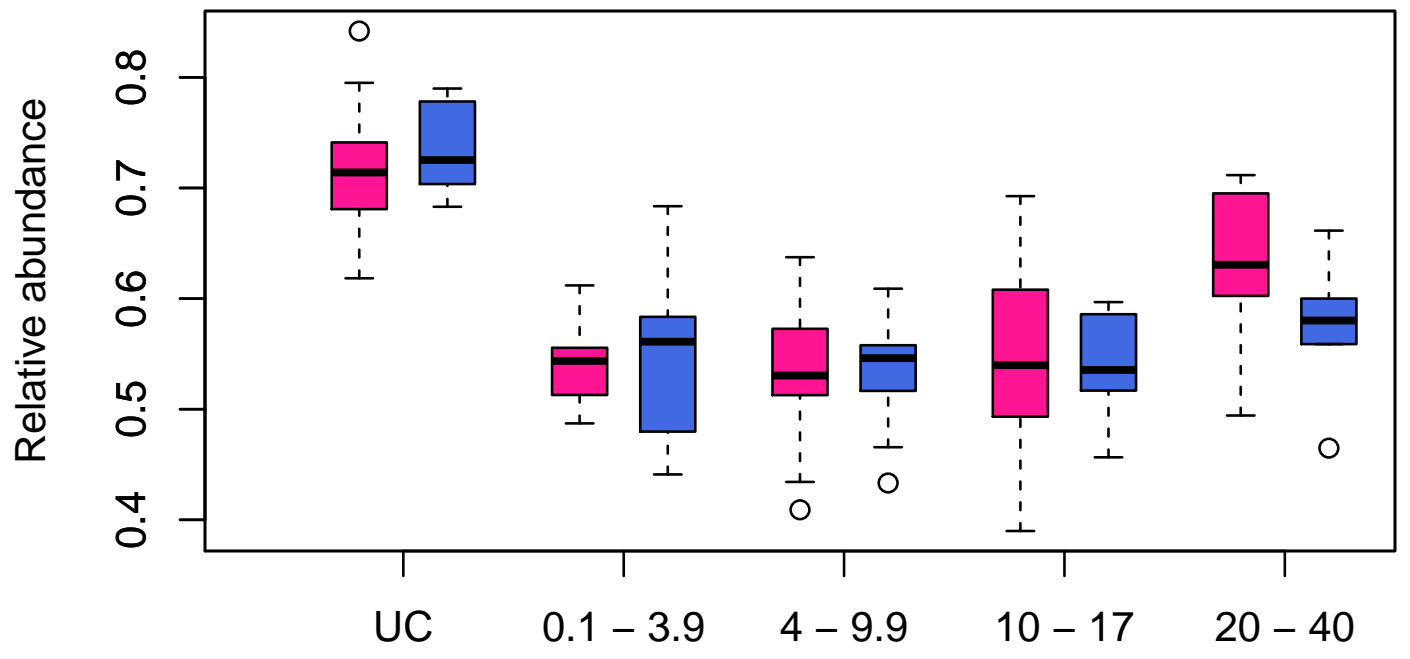

Age class (years) 
IgG1_A2F0G

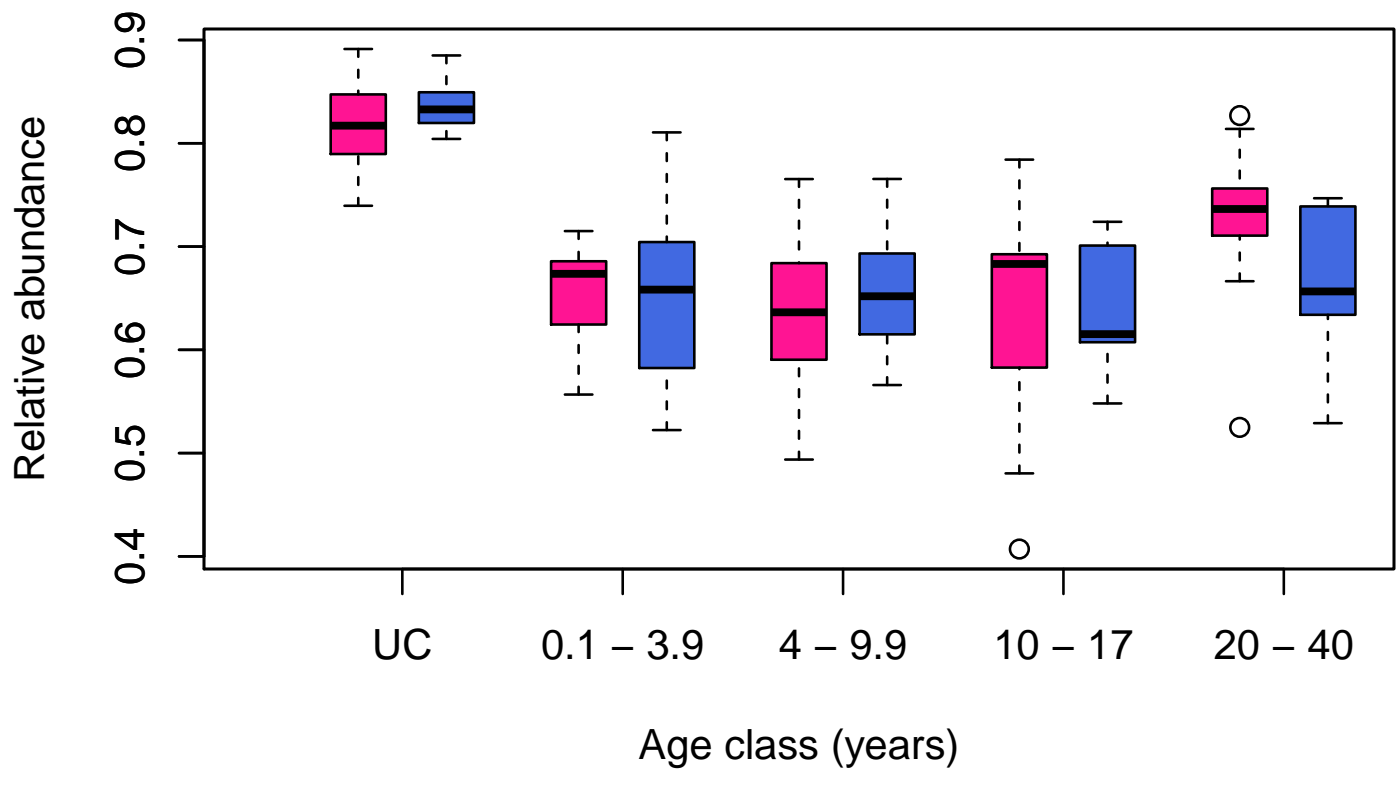

IgG1_A2FG

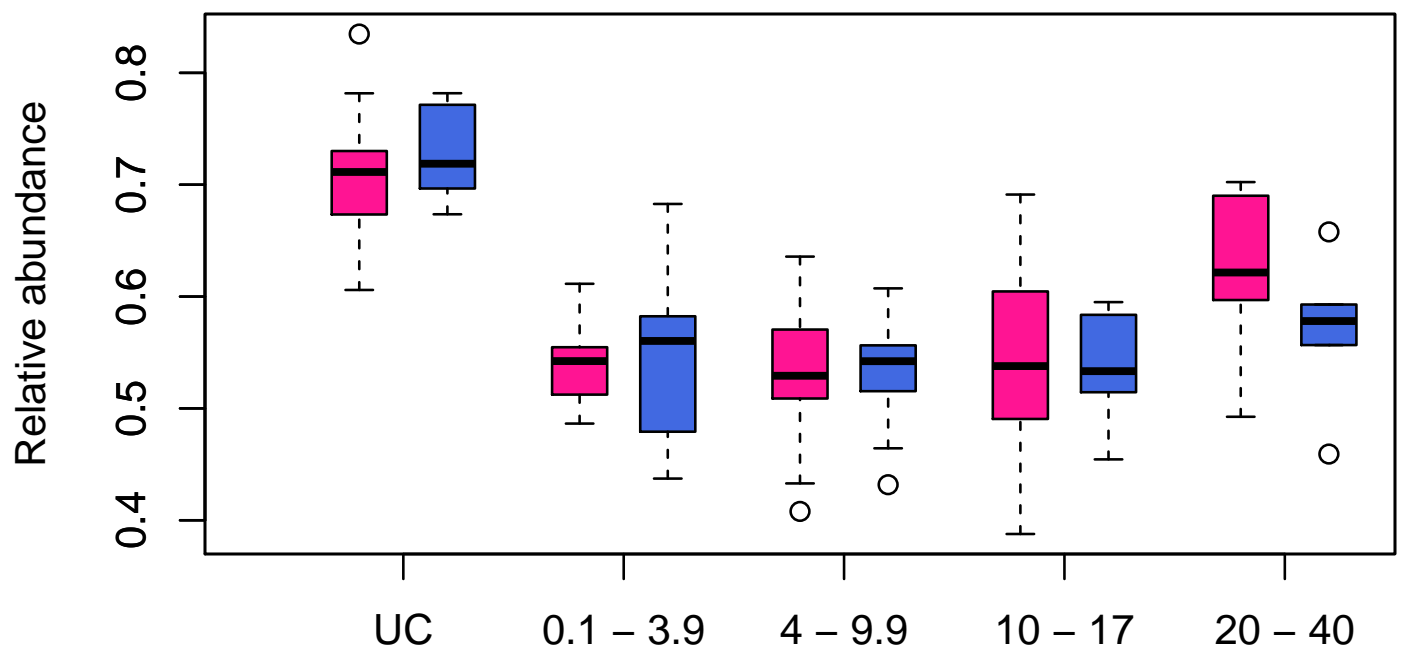

Age class (years) 


\section{IgG1_A2D}

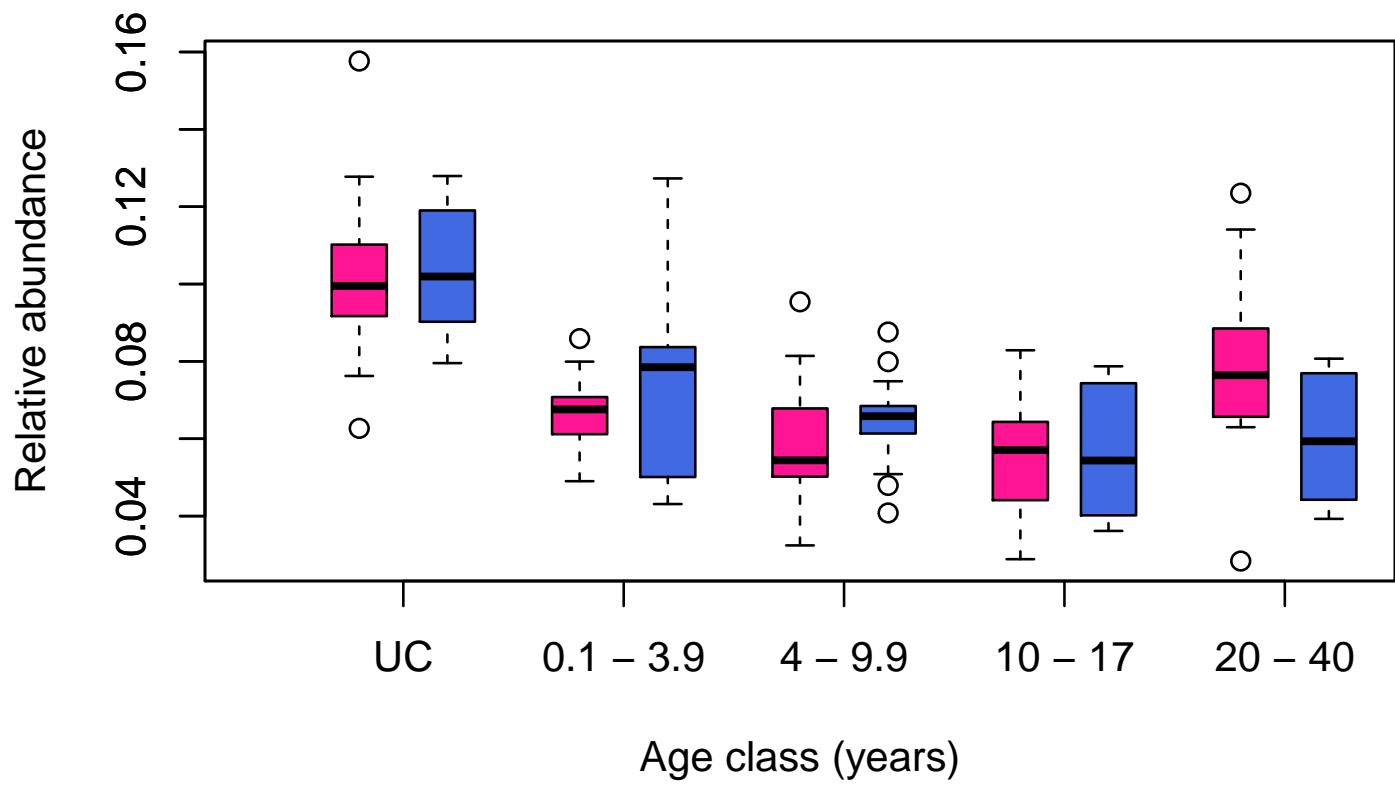

IgG1_A2F0D

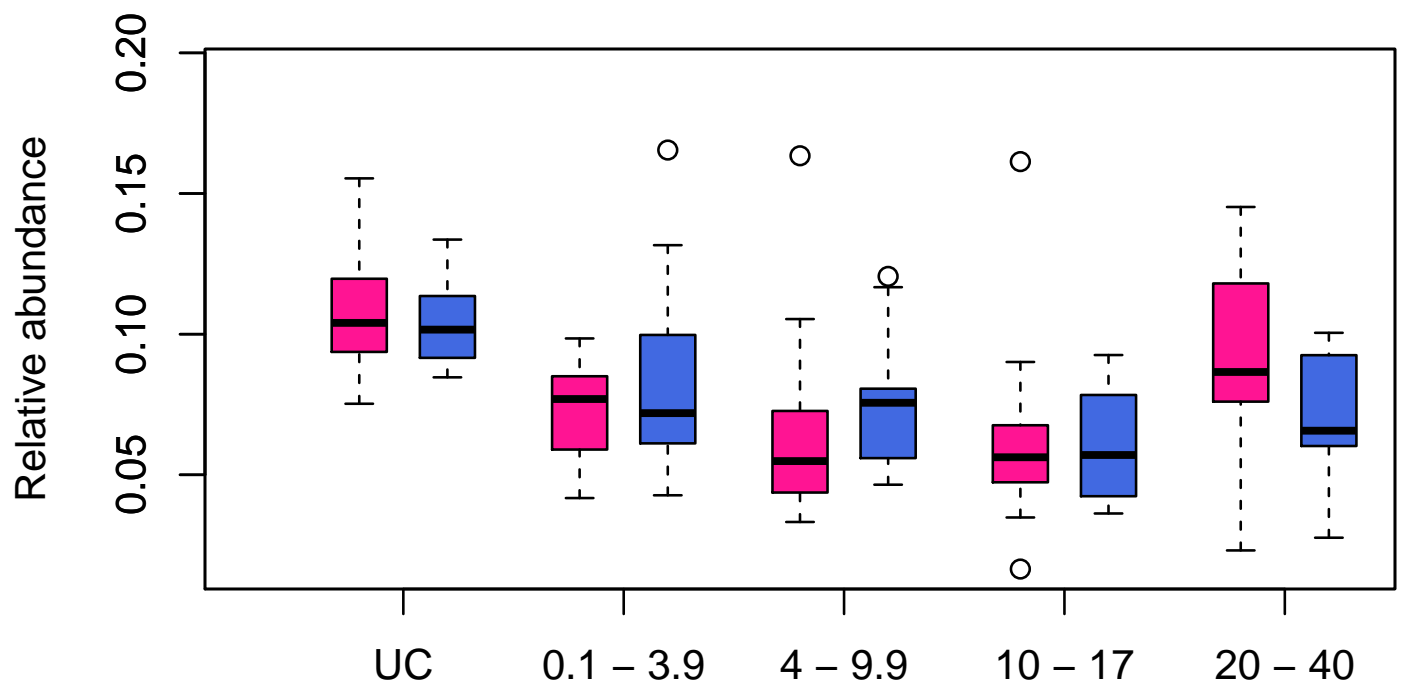

Age class (years) 


\section{IgG1_A2FD}

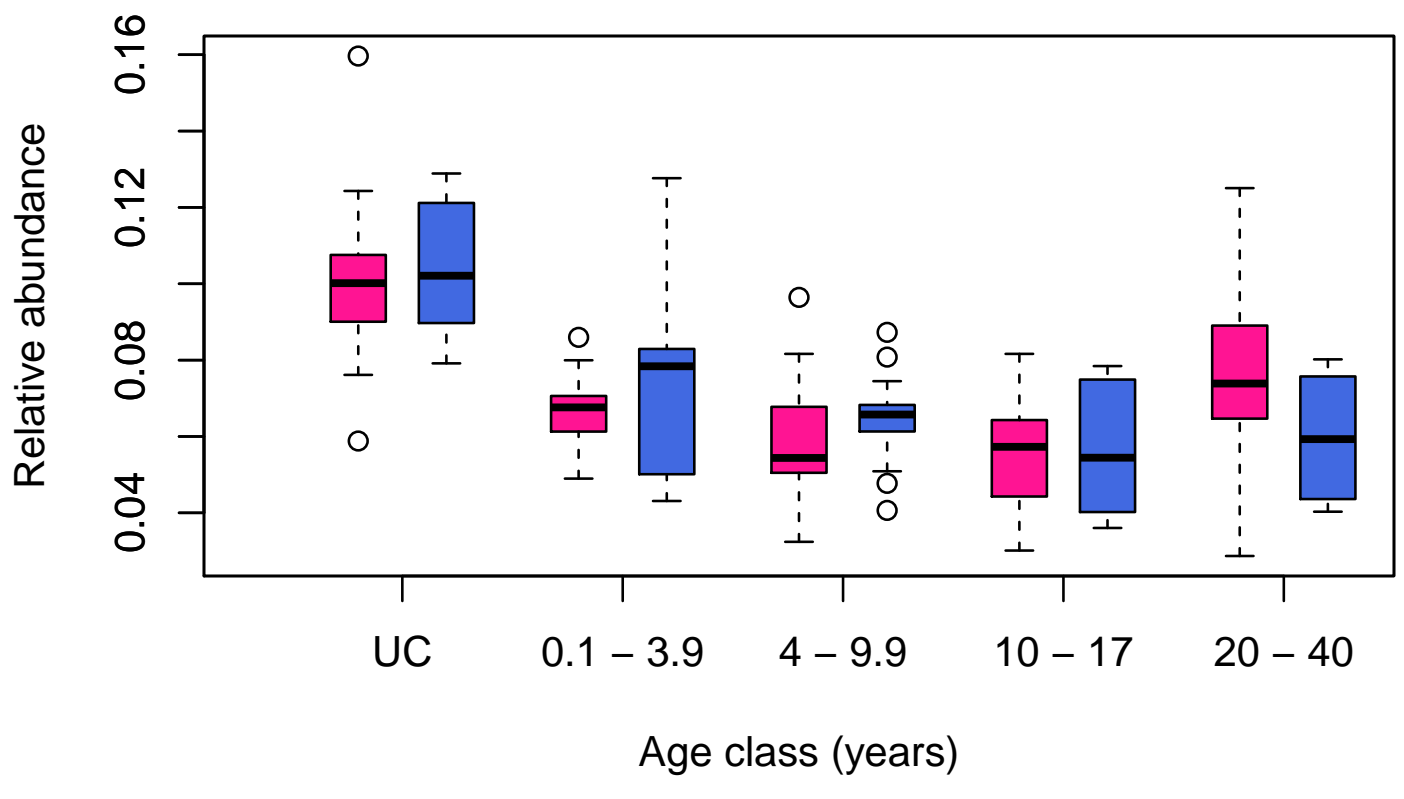

IgG1_A2GD

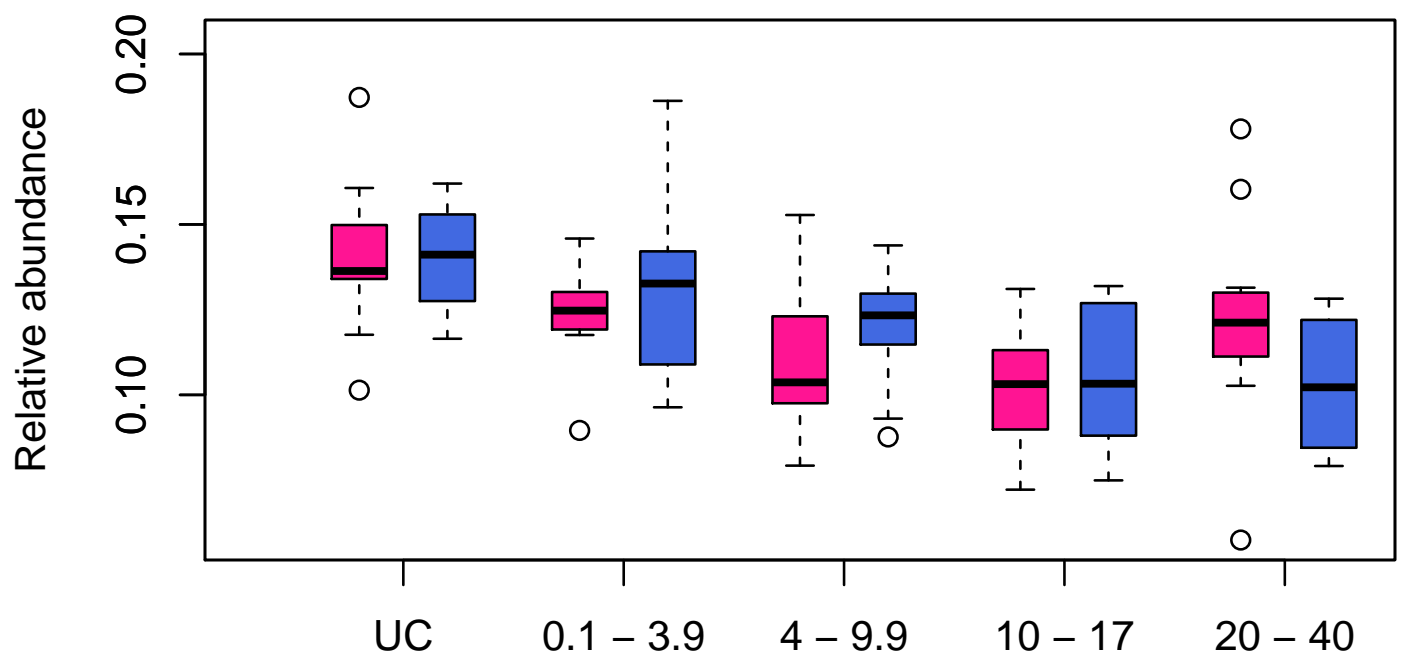

Age class (years) 
IgG1_A2FD_B

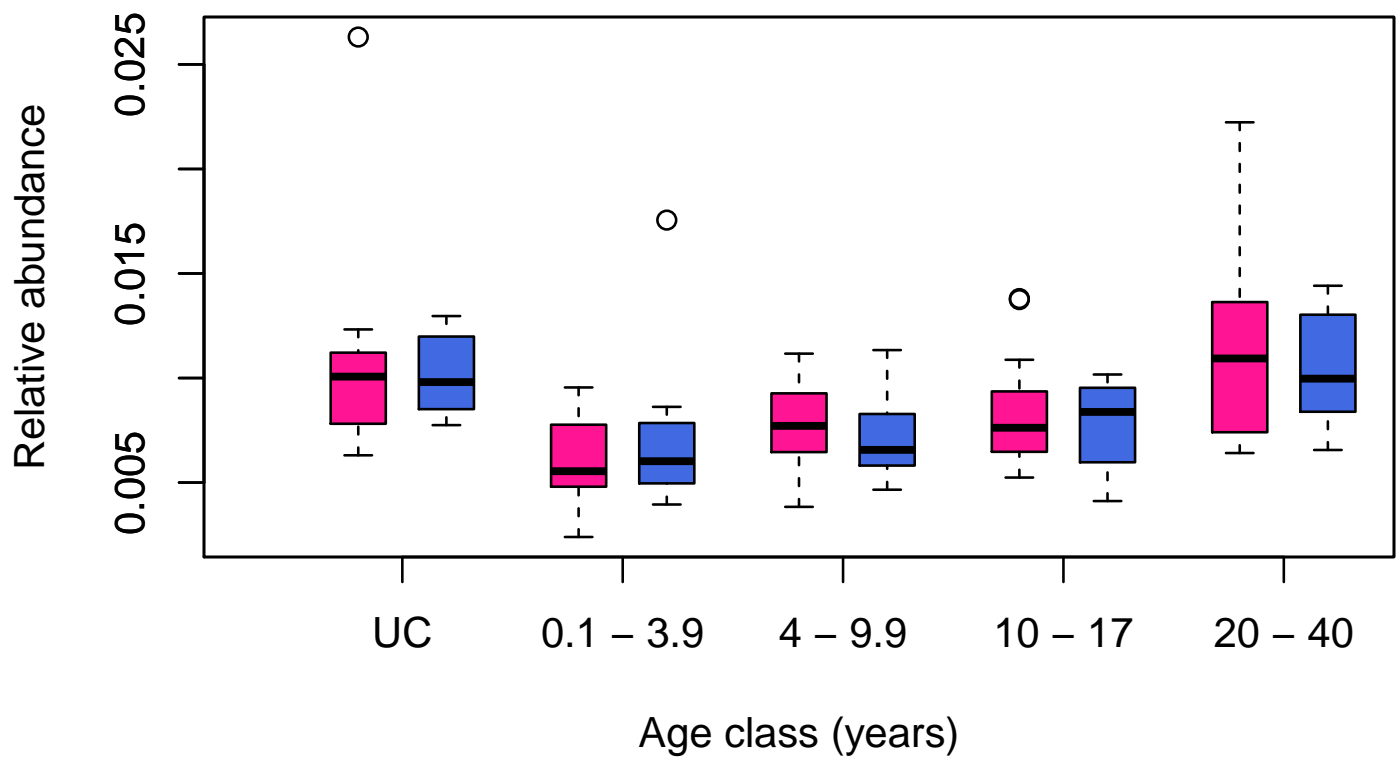

IgG1_agal

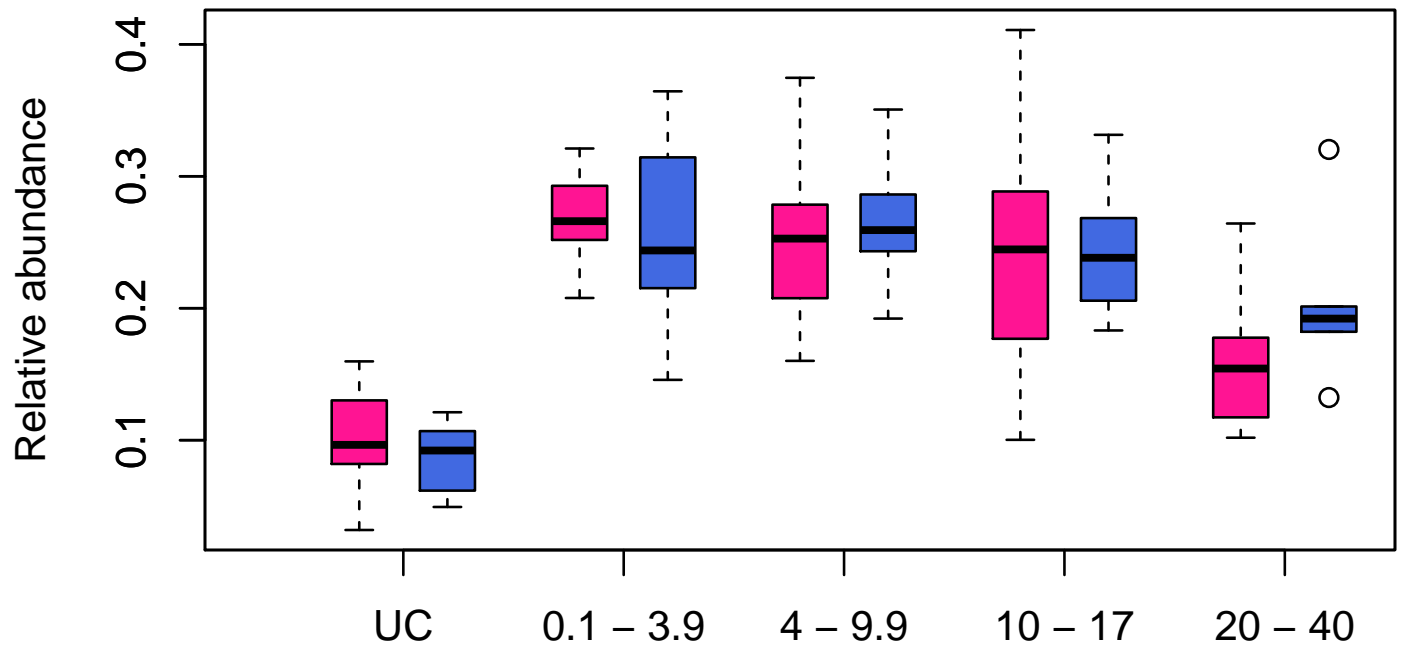

Age class (years) 
IgG23_A2FG

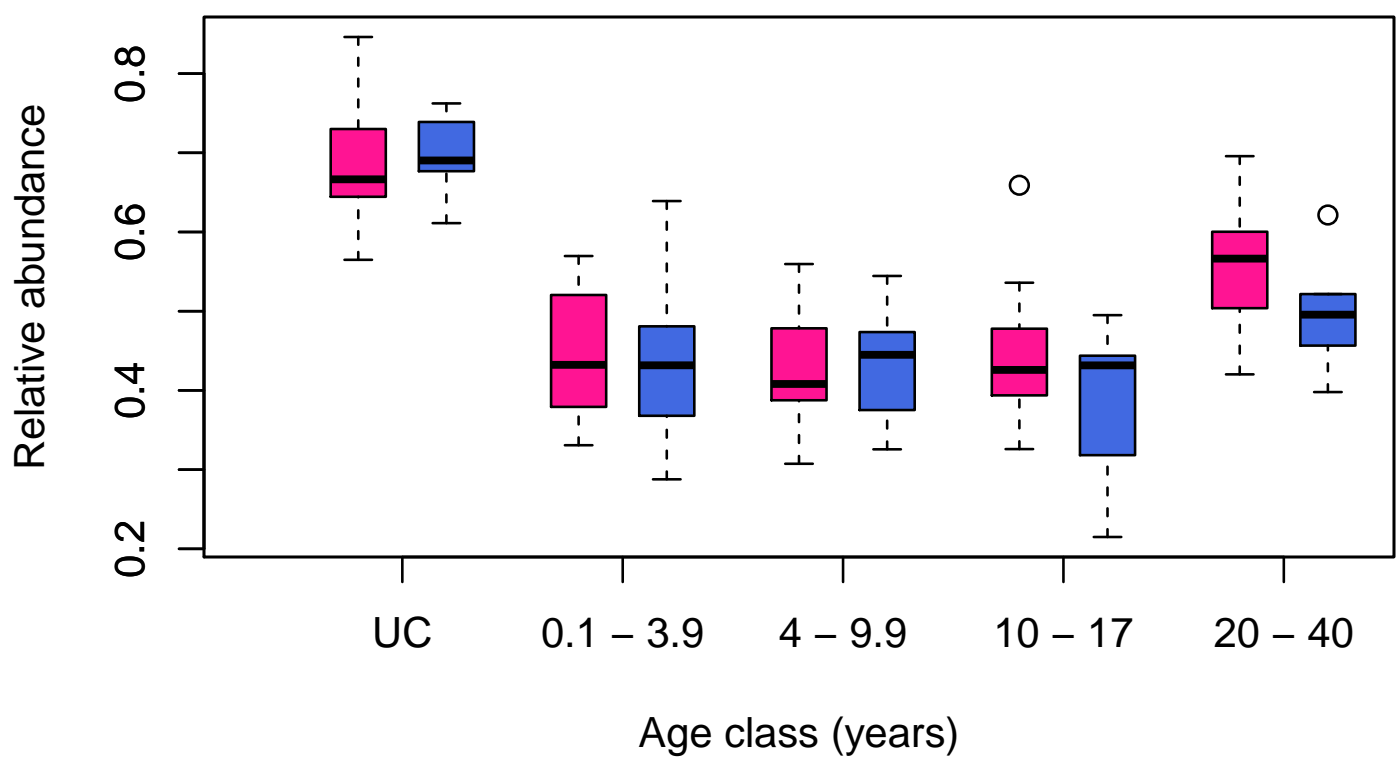

IgG23_A2FD

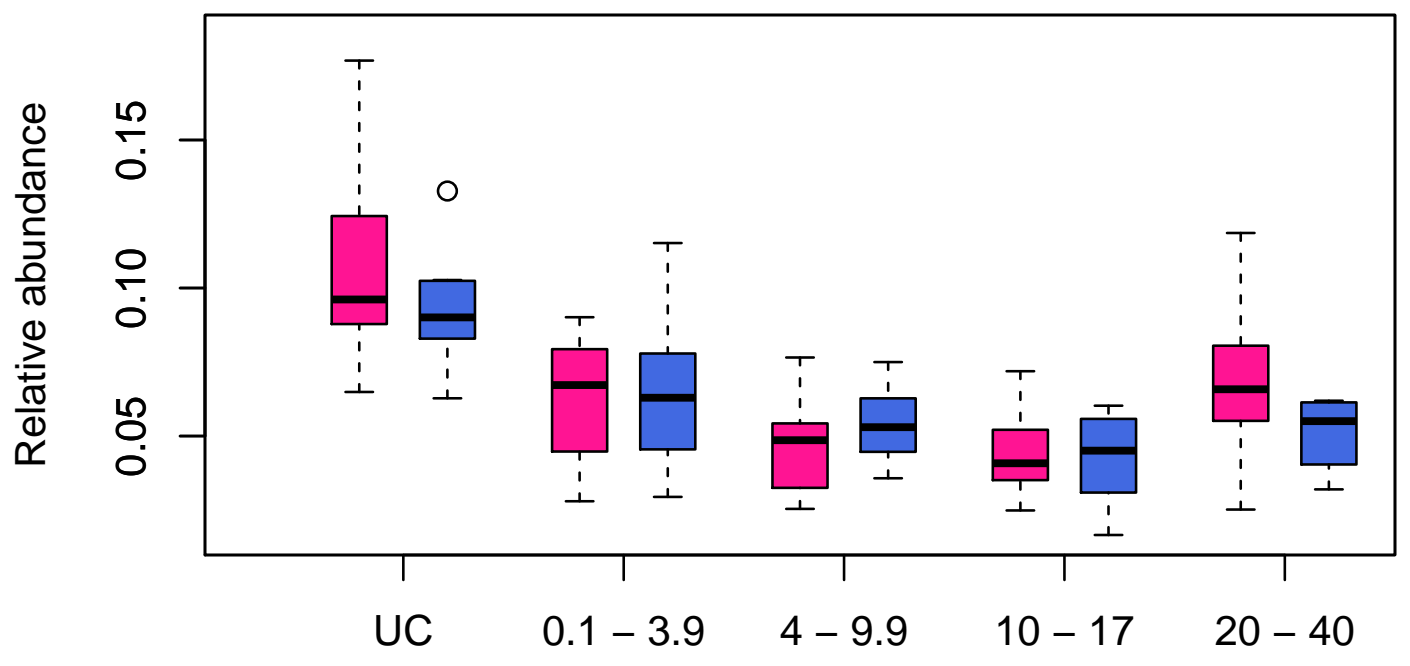

Age class (years) 
IgG23_A2FGD

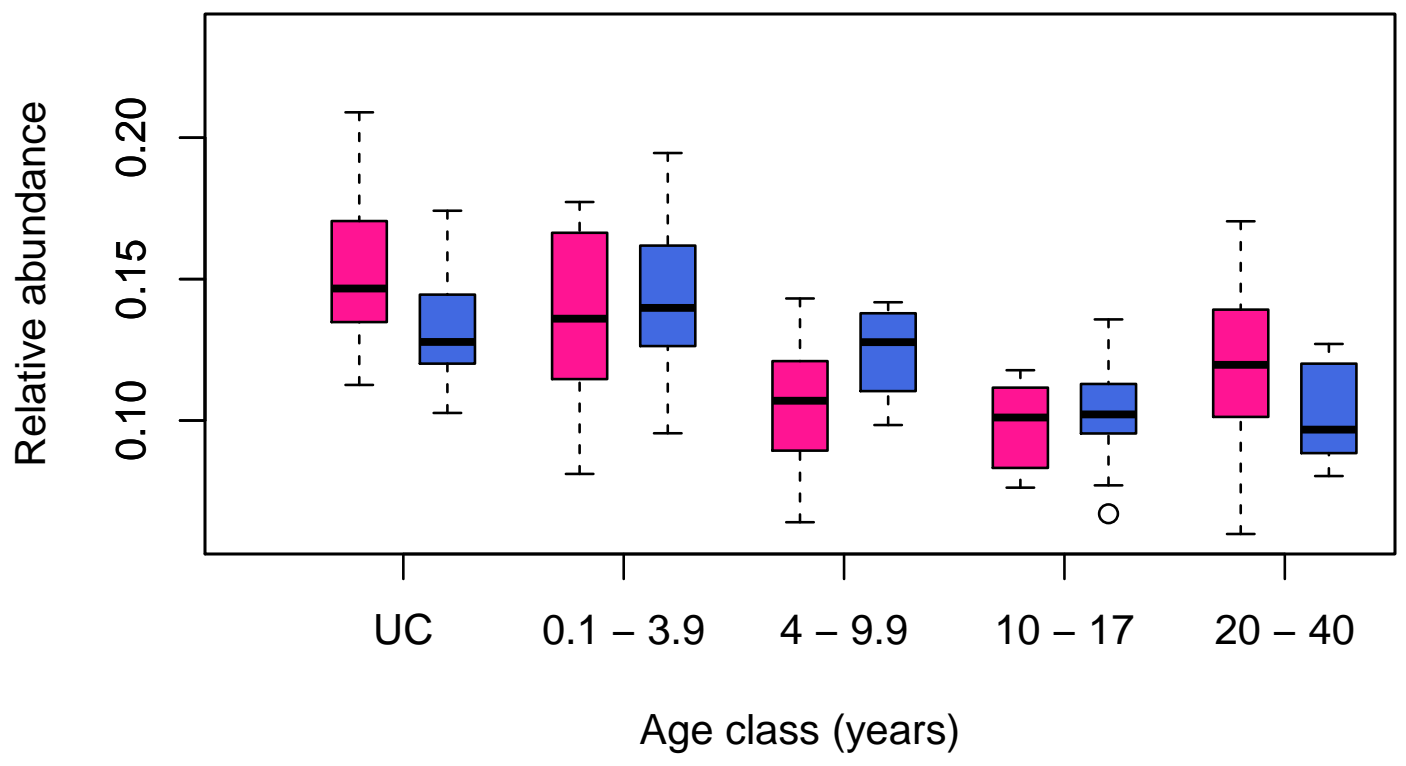


Figure S-5 Levels of all glycans and derived glycan traits of IgG1 and $\operatorname{lgG} 2 / 3$ per age class. Male and female samples were combined. UC: samples taken from the umbilical cord.

\section{IgG1_H3N3F1}

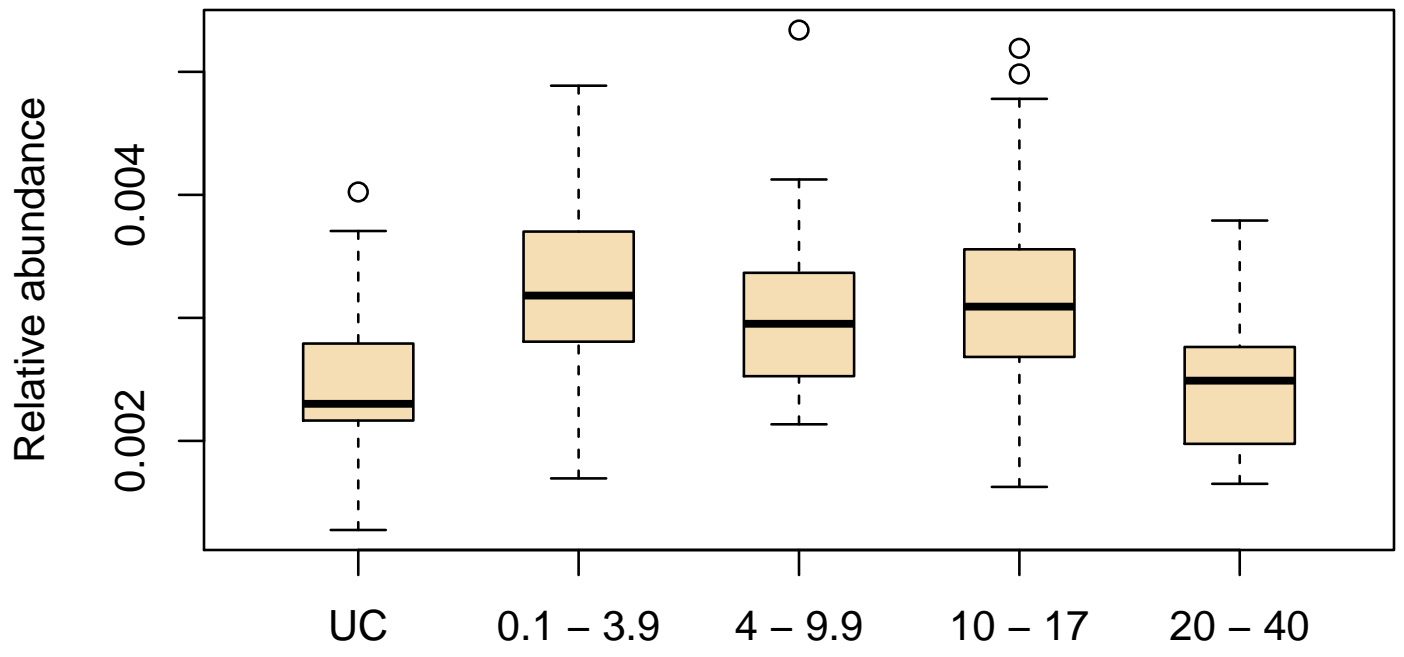

Age class (years) 
IgG1_H3N4

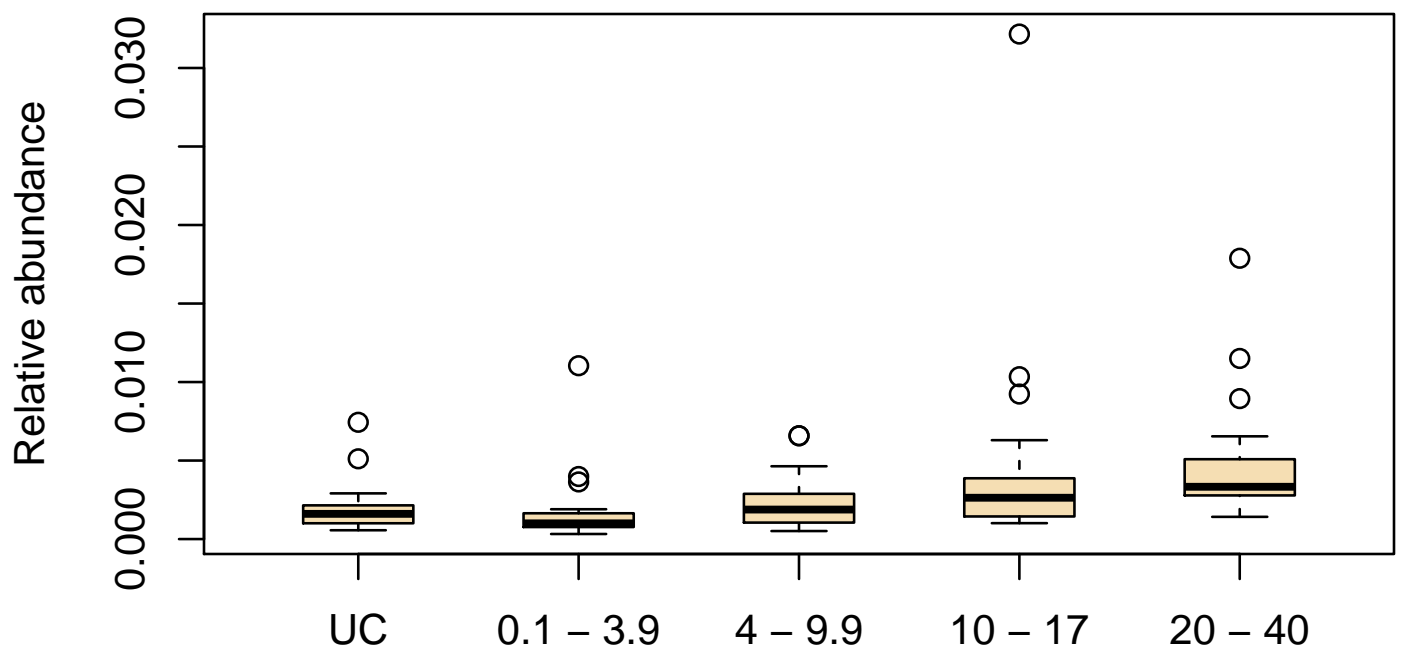

Age class (years)

IgG1_H4N3F1

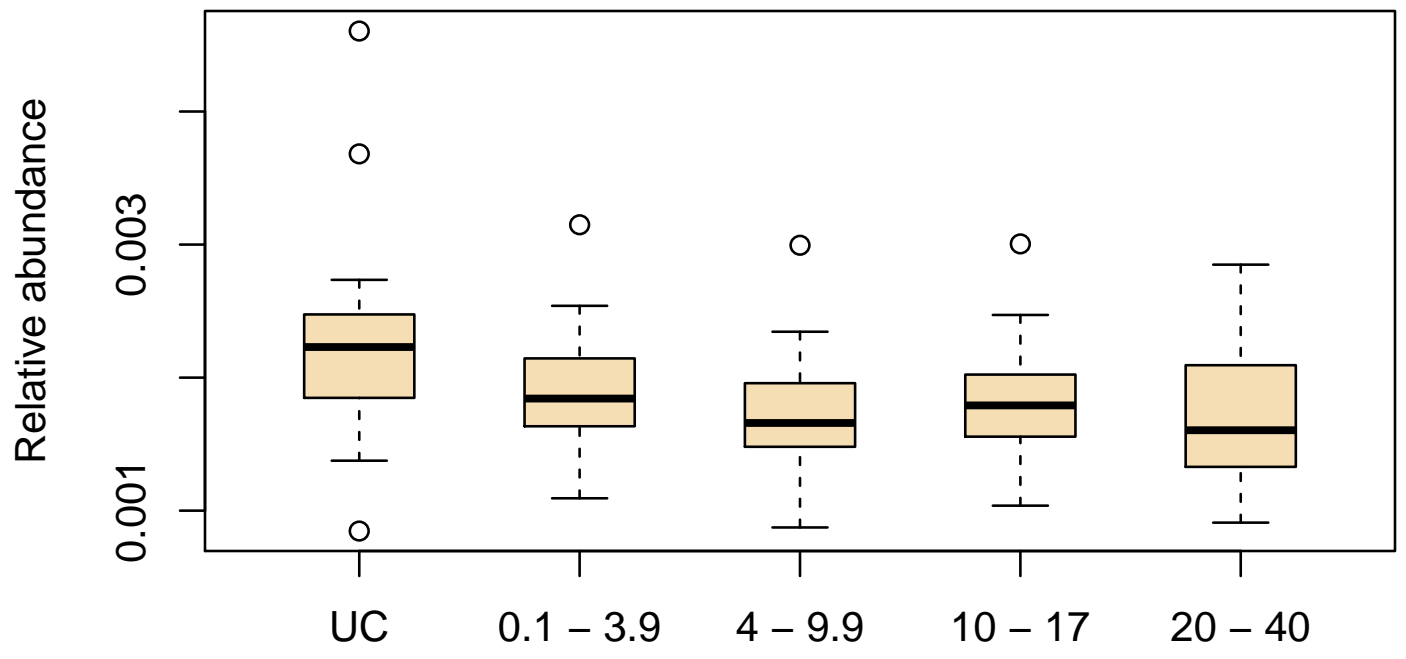

Age class (years) 
IgG1_H3N5F1

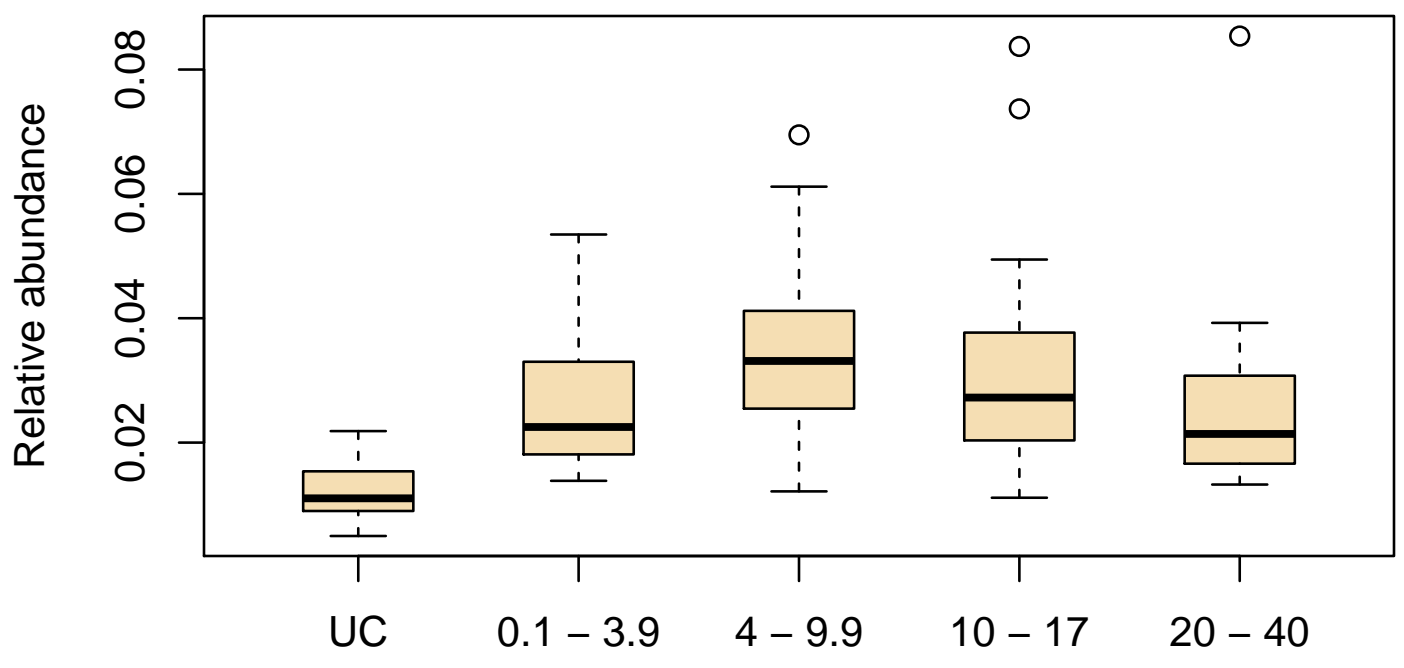

Age class (years)

IgG1_H4N5

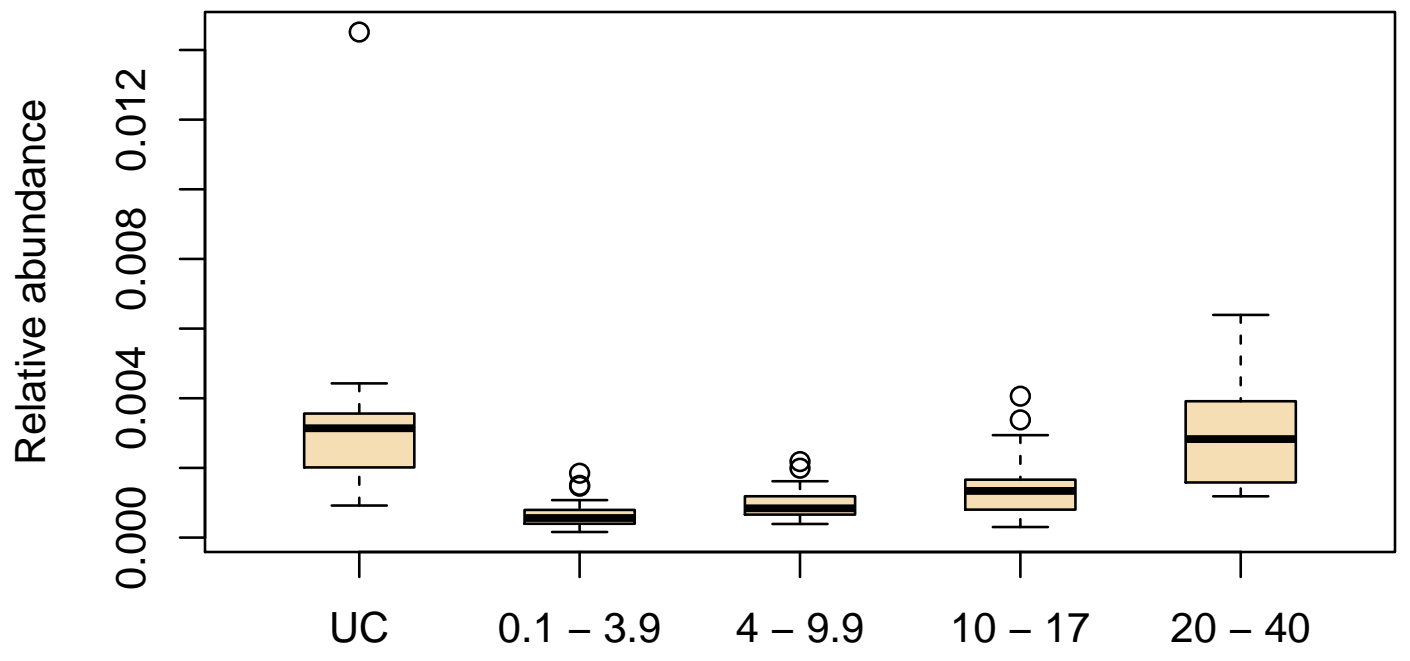

Age class (years) 
IgG1_A2

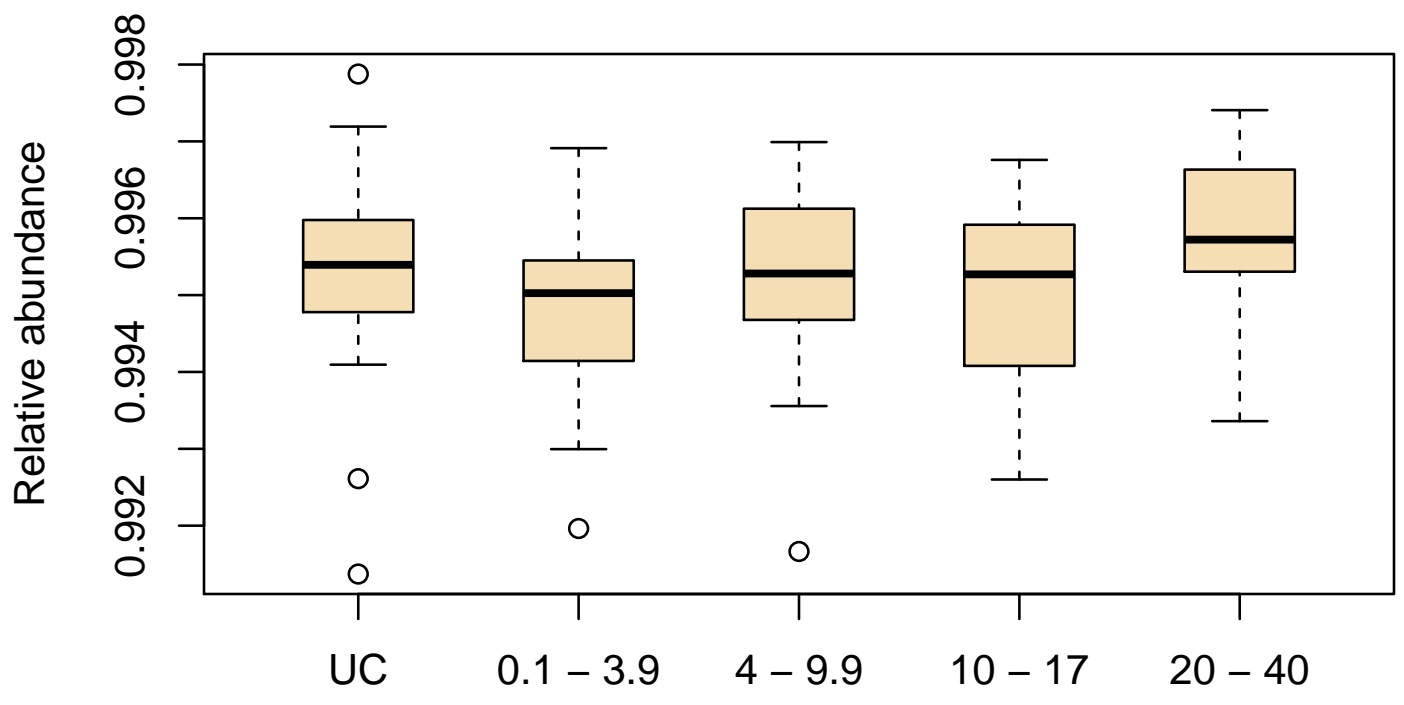

Age class (years)

IgG1_B

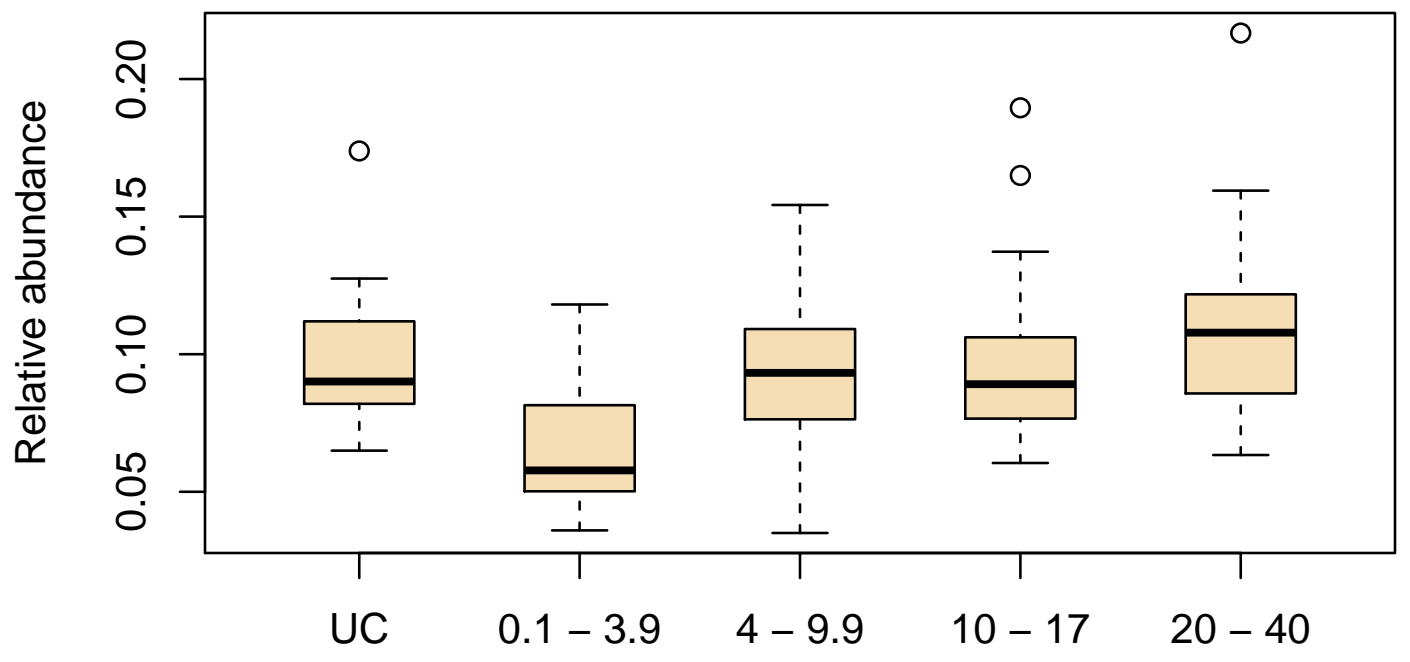

Age class (years) 
IgG1_A2F0G

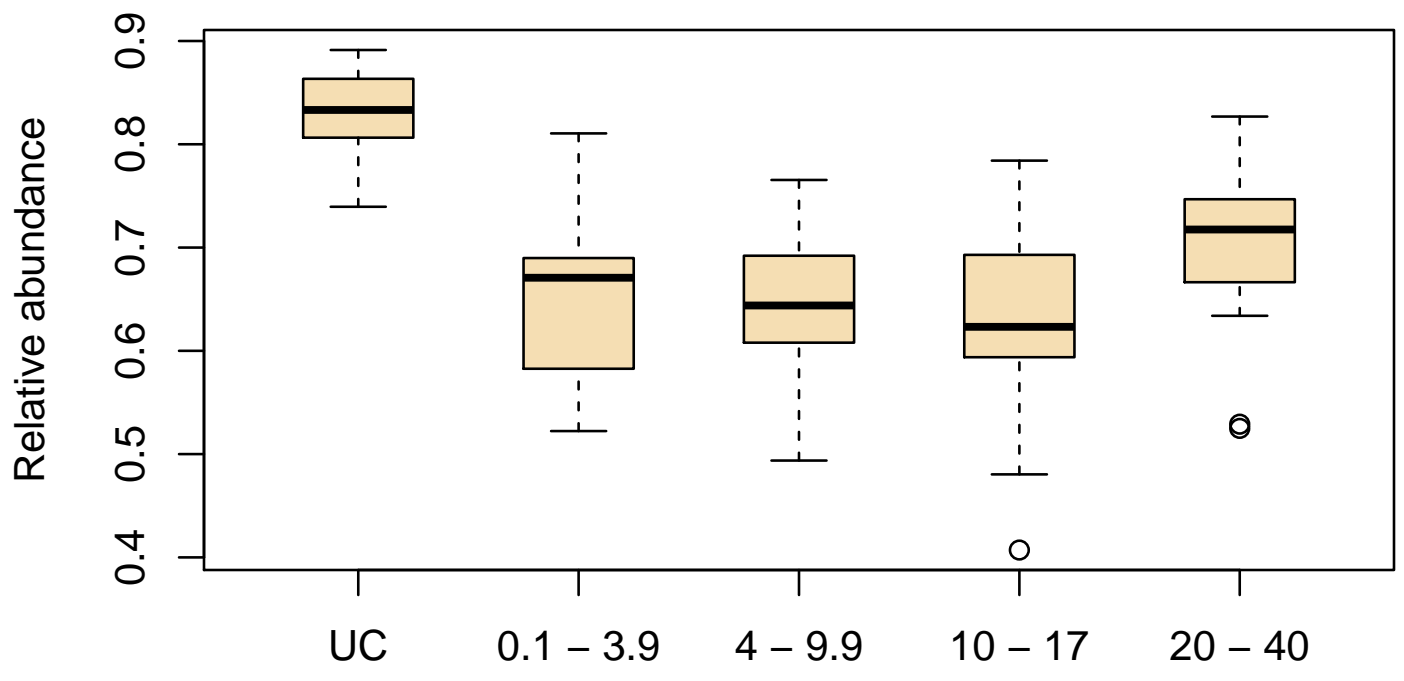

Age class (years)

IgG1_A2FG

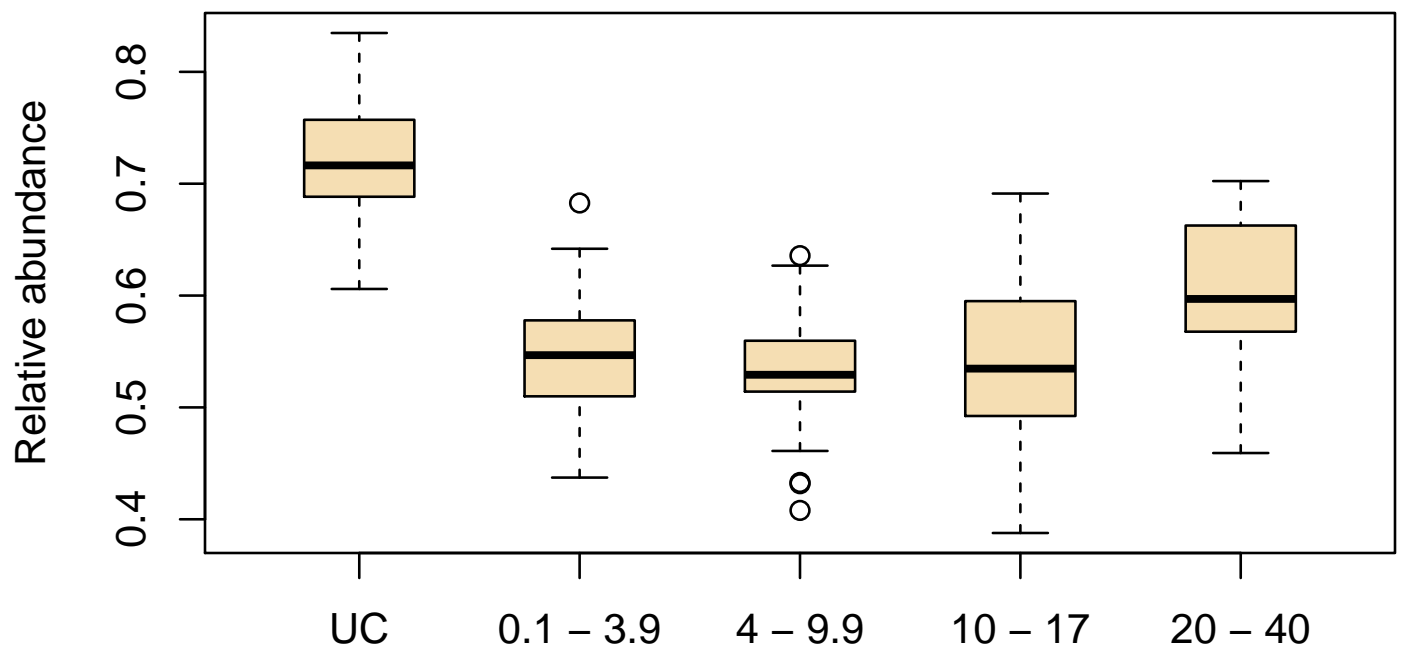

Age class (years) 
IgG1_A2FD

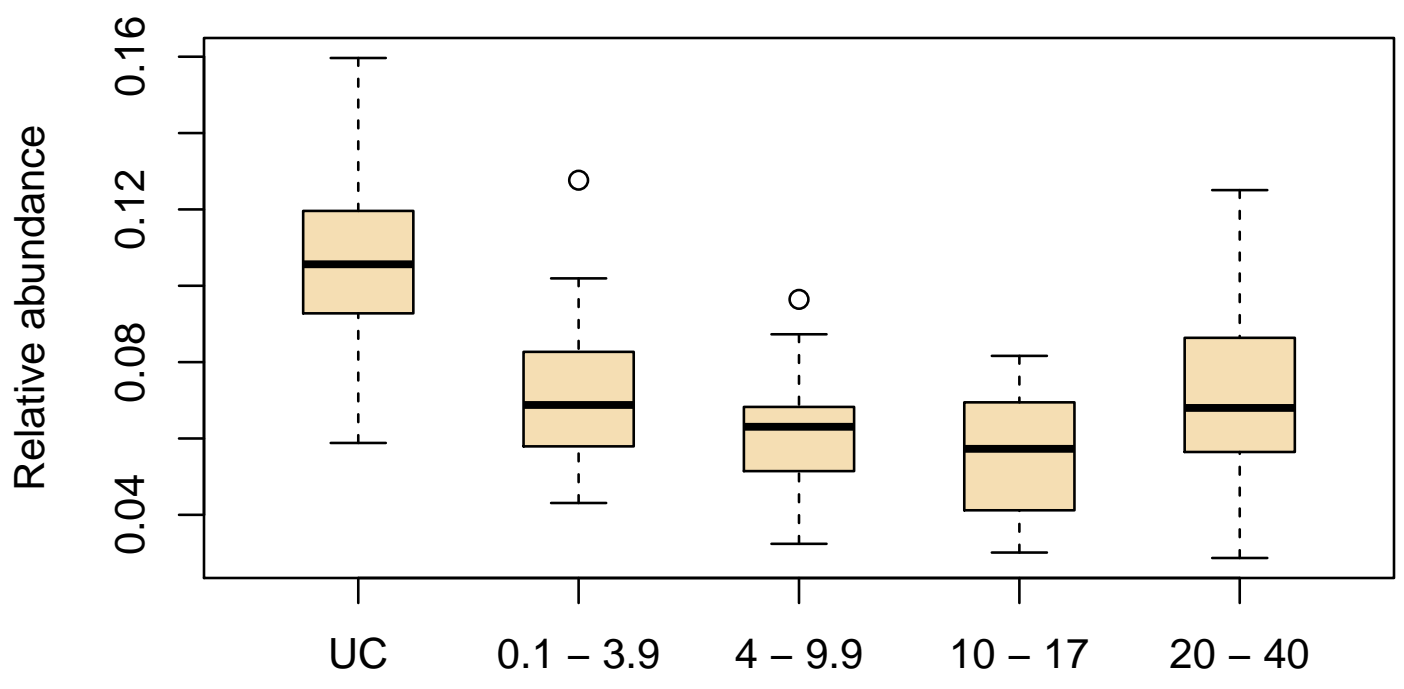

Age class (years)

IgG1_A2GD

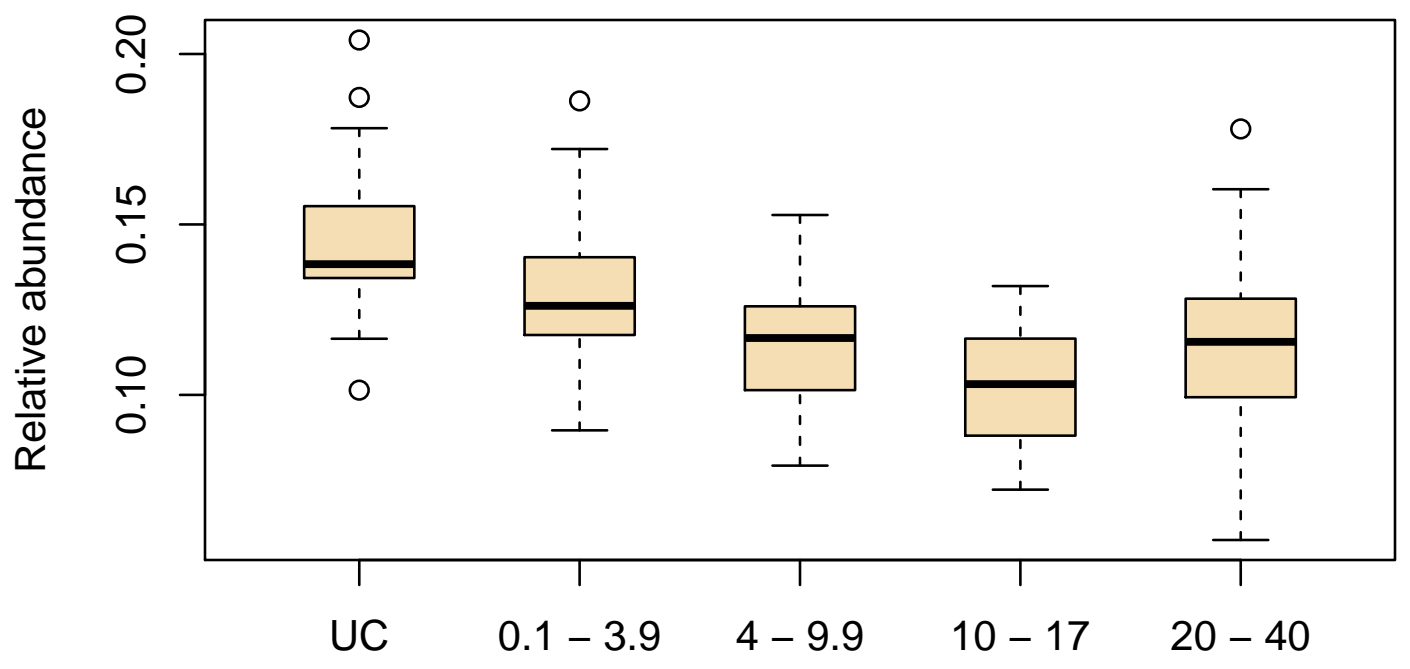

Age class (years) 
IgG1_A2FD_B

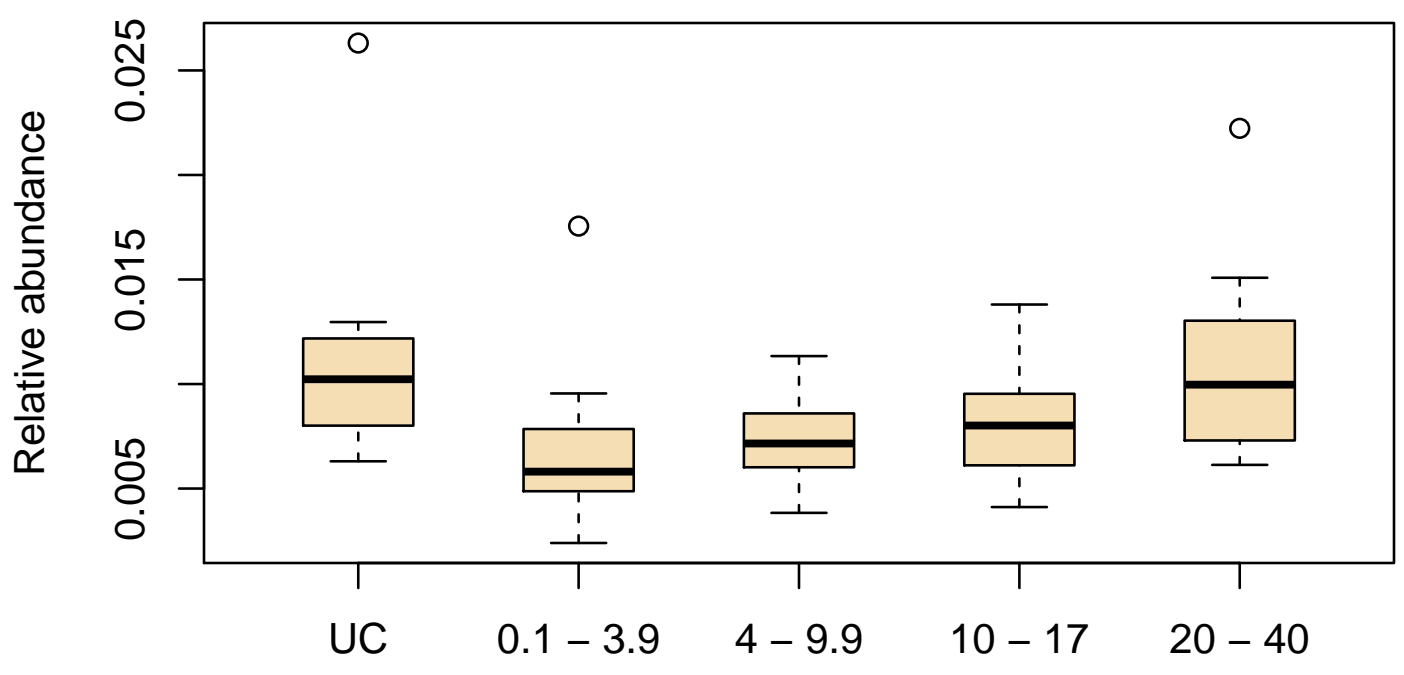

Age class (years)

\section{IgG1_agal}

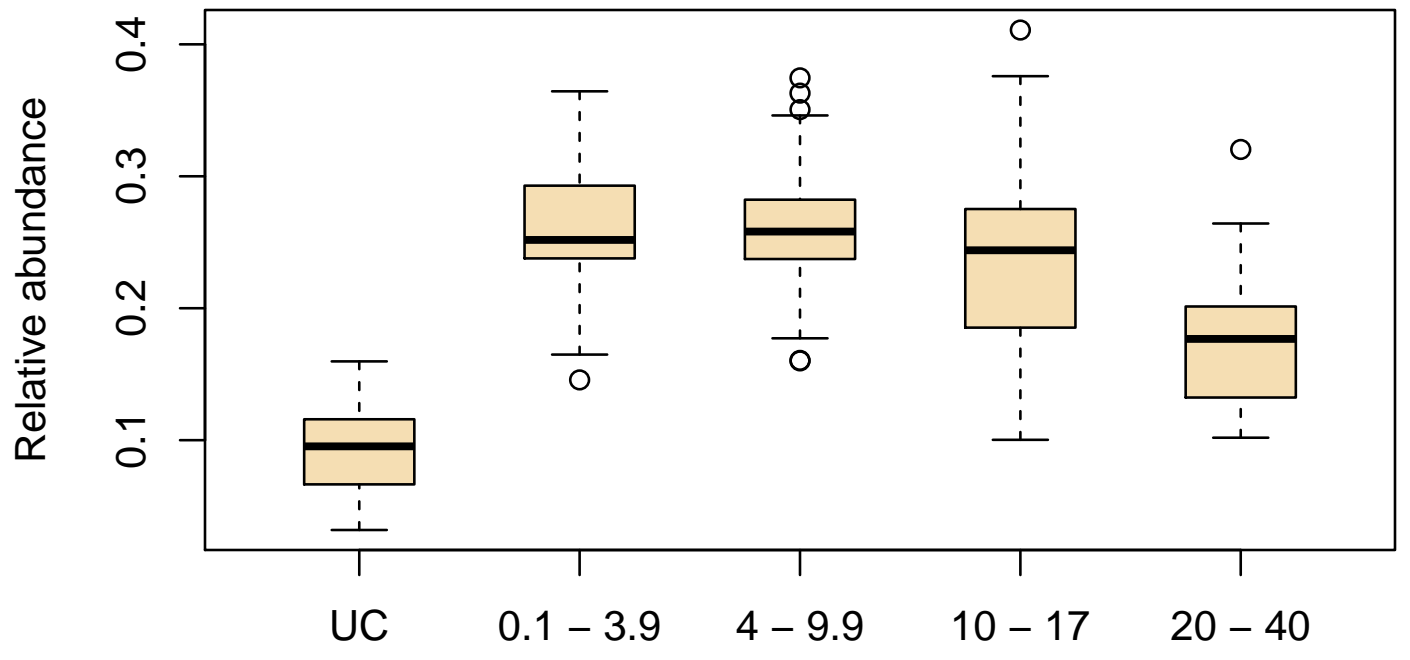

Age class (years) 
IgG23_A2FG

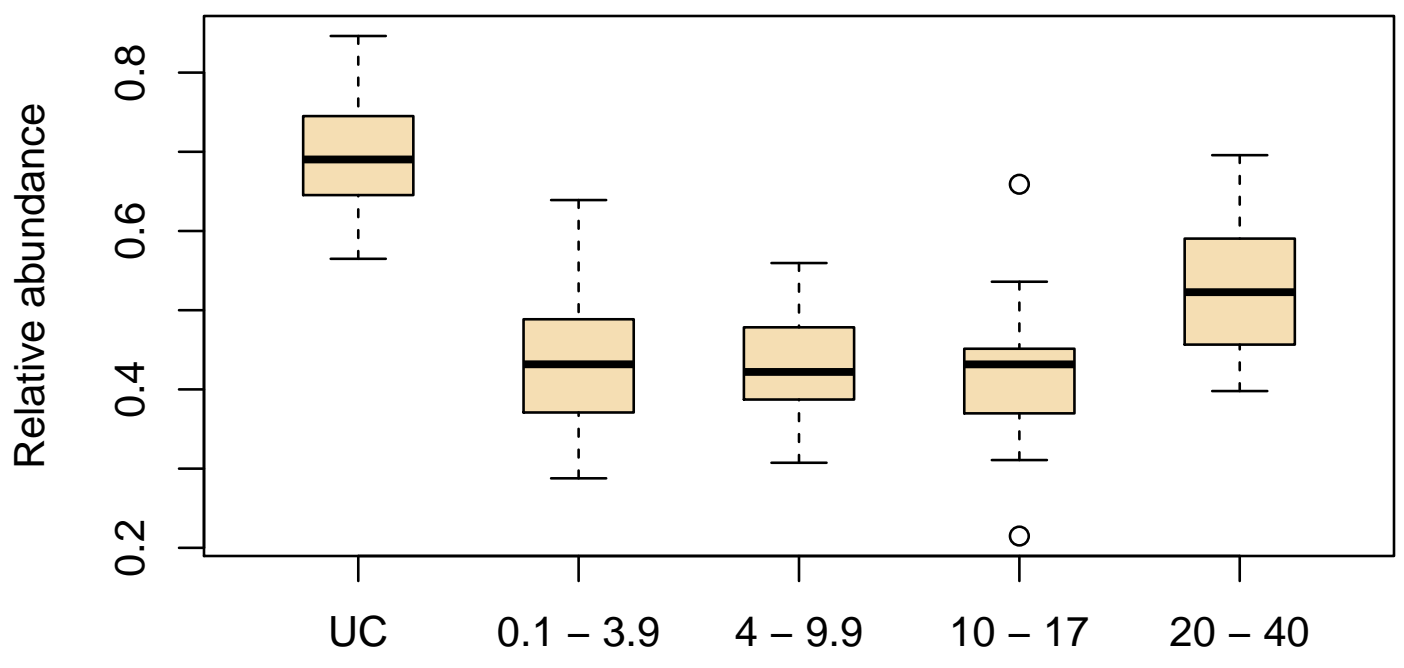

Age class (years)

IgG23_A2FD

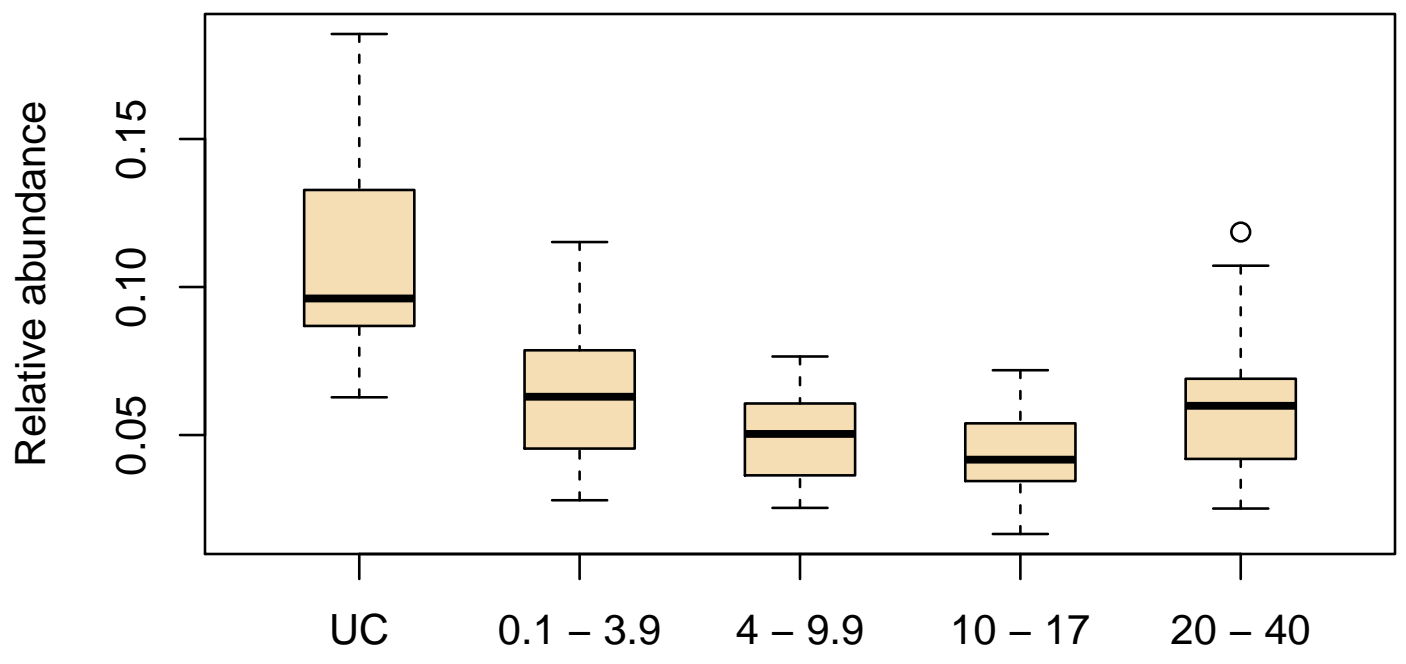

Age class (years) 
IgG23_A2FGD

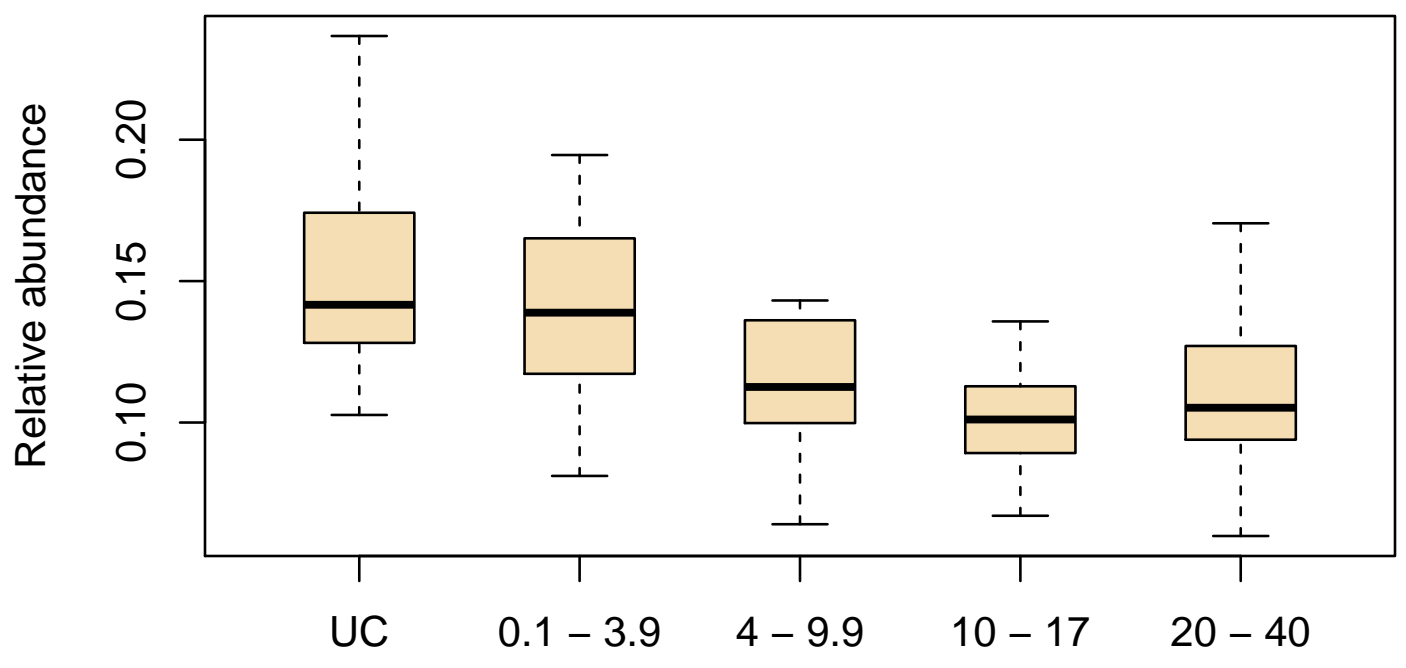

Age class (years) 